\title{
Prenatal stress alters fear-conditioned behaviors and the response to serotonergic drugs
}

\author{
William C. Griffin III \\ West Virginia University
}

Follow this and additional works at: https://researchrepository.wvu.edu/etd

\section{Recommended Citation}

Griffin, William C. III, "Prenatal stress alters fear-conditioned behaviors and the response to serotonergic drugs" (2001). Graduate Theses, Dissertations, and Problem Reports. 1432.

https://researchrepository.wvu.edu/etd/1432

This Dissertation is protected by copyright and/or related rights. It has been brought to you by the The Research Repository @ WVU with permission from the rights-holder(s). You are free to use this Dissertation in any way that is permitted by the copyright and related rights legislation that applies to your use. For other uses you must obtain permission from the rights-holder(s) directly, unless additional rights are indicated by a Creative Commons license in the record and/ or on the work itself. This Dissertation has been accepted for inclusion in WVU Graduate Theses, Dissertations, and Problem Reports collection by an authorized administrator of The Research Repository @ WVU.

For more information, please contact researchrepository@mail.wvu.edu. 
Prenatal Stress Alters Fear-Conditioned Behaviors and the Response to Serotonergic Drugs

\title{
William C. Griffin III
}

Dissertation submitted to the School of Medicine

At West Virginia University

In partial fulfillment of the requirements

For the degree of

Doctor of Philosophy

Dale L. Birkle, Ph.D., Chair

Kristine Krajnak, Ph.D.

Adrienne K. Salm, Ph.D.

David Schaal, Ph.D.

David J. Smith, Ph.D.

David A. Taylor, Ph.D.

Department of Pharmacology and Toxicology

\author{
Morgantown, West Virginia \\ 2001
}

Keywords: Prenatal Stress, Corticotropin Releasing Factor (CRF), Serotonin, Acoustic Startle, Fear-potentiated Startle, Conditioned Freezing 


\begin{abstract}
Prenatal Stress Alters Fear-Conditioned Behaviors and the Response to Serotonergic Drugs
\end{abstract}

\title{
William C. Griffin III
}

Chronic, inescapable stress during pregnancy (i.e. prenatal stress) modifies the behavior of the adult offspring. Several behaviors were evaluated in adult prenatally stressed (PS) rats, most notably the acoustic startle response and behavioral inhibition (i.e. freezing). PS rats do not differ in baseline peak startle responding, or in the habituation of the peak startle response when compared to the non-prenatally stressed (CON) rats. However, PS rats have greater peak responses and longer latencies to reach the peak response following systemic doses of the 5- $\mathrm{HT}_{1 \mathrm{~A}}$ agonist, 8-OH-DPAT, compared to CON rats. The PS rats had shorter latencies than the $\mathrm{CON}$ rats under vehicle treatment.

In fear-potentiated startle testing, peak startle responding did not reach the same levels in the PS rats as it did in the CON rats; thus, it was inhibited. Latencies were depressed during fear-potentiated startle testing compared to baseline testing, and the PS rats had generally shorter latencies than the CON rats. Additionally, the latency was more sensitive to context after the training, demonstrating a decrease during the leader trials of the test session, before the increase in peak startle was noted during the testing trials. In defensive freezing, PS rats displayed more freezing following acute footshock and 24 hours later in the same context when the footshock was not presented. Rears were markedly attenuated immediately post-shock and, the next day, and only recovered significantly in the CON rats.

The data presented are consistent with PS rats being more reactive to fear provoking stimuli. The ramifications of this are discussed in terms of the neural circuitry that underlie the behaviors with possible changes that might be induced by prenatal stress. Additionally, some observations regarding the contextual influence on the acoustic startle response and freezing are discussed. Finally, several pieces of evidence are presented demonstrating that gross developmental and some neurochemical parameters of PS rats are indistinguishable from $\mathrm{CON}$ rats from birth to adulthood suggesting that source of the behavioral differences noted above is subtle in nature. 
In memory of my Grandparents

Gertrude Fereday Koebig

Philip Walter Koebig, Jr.

Eleanor Waters Griffin

And my high school science teacher, Churchill Briley 


\section{ACKNOWLEDGEMENTS}

First and foremost on the list of acknowledgements is my advisor, Dr. Dale Birkle. I have had the great fortune to explore and learn about the discipline of pharmacology in her laboratory. Over the years, she has taught me a great deal about science and the politics of science. I have grown tremendously, intellectually and personally, while working in her laboratory. Dr. Birkle sincerely cares about teaching and about the progress of all of her students. She teaches at many levels: graduate, professional, undergraduate and through her involvement with AWIS, even teaches at the junior high level! In my long educational journey, Dr. Birkle has the distinction of being the only lecturer, out of many that I have witnessed, to receive a spontaneous round of applause from a class after what was a remarkably well-delivered and eloquent lecture. To me, that experience speaks volumes about her ability to engage students and educate them, even when she only has 50 minutes to do it. I believe that Dr. Birkle's ability to teach comes from her positive outlook on life, her down-to-earth attitude and her desire to have the best for her students. Outside of the classroom, Dr. Birkle is a genuine, caring person who takes great pleasure in cultivating friendships and exploring what life has to offer. Dr. Birkle is truly an asset to the School of Medicine and the University.

Drs. Dave Schaal, Adrienne Salm, Kristine Krajnak, Dave Taylor and Dave Smith have all been an valuable part of my educational experience by serving on my dissertation committee. They are a formidable and challenging group, to say the least! All of them have contributed in a significant way to my project and my training. I am particularly indebted to Drs. Taylor and Smith who served as surrogate advisors while Dr. Birkle was 
away on sabbatical and even while she was here. They both had the misfortune of having offices located in close proximity to Dr. Birkle's lab.

Throughout my years at WVU, I have met quite a number of people that have given me good advice, good conversation and sometimes both. Drs. Knox Van Dyke, Mark Reasor and Mike Mawhinney stand out as three faculty members who really seemed to take an interest in me and my progress as a developing scientist. I am grateful for their advice and good conversation over the years. My fellow students, Dave White (now Dr. Dave), Mike Taylor (now Dr. Mike), Karen Rust, Erin Sikora, Stacy Brower and Heath Skinner, have provided with me many good times and memories. And, on occasion, we talked about pharmacology or even, heaven forbid, toxicology! I owe Dave special thanks for being a good friend as well as taking time out his schedule to get me started in the lab. Likewise, Mike has been a good friend and deserves a hearty "thank you" for piloting the boat as we searched for fish in a fishless lake! I sincerely hope that all of our friendships continue over the years.

These days completing a dissertation project is really a team effort. I have been fortunate to have been surrounded by a great group of people. Marshall Krouse, Wendy Webster and Brent Shaffer have all moved on to better things but were there when I first started and helped me out tremendously. A new group has taken over and continues to provide expertise in the lab. Tony DeBastiani, Brent Lally, Michal Kraspulski and Molly Ford, are proving to be quite valuable additions to the lab. A special thanks is also due to Dr. Adrienne Salm. Without her expertise in neuroanatomy, the current grant funding the lab would not be possible and, of course, neither would hiring good people to do the work! 
Two people deserve to be singled out for their behind-the-scenes contributions to my project, Dreama Lemley and Chris Van Dyke. The projects in our lab involve using many rats and Dreama has taken care of our rats for a number of years. She has helped me in a number of valuable ways to ensure that my rats were happy and my projects progressed smoothly. Dreama is a great person and deserves much credit for the work she does in the Animal Care facility.

Chris Van Dyke is our resident computer and electronics guru. He has provided me with a lot of support and a lot of good conversation over the years. A number of times, Chris has stopped what he was doing to answer questions or to help me with equipment malfunctions so that an ongoing experiment would not be compromised. Chris is a fantastic person who really enjoys his job and the people he works with. He is truly an asset to the School.

No list of acknowledgements would be complete without mentioning my family. They have endured the many years of my educational journey and, unfortunately for them, there are many years left (we never stop learning do we!). My parents, William and Jane Griffin have always encouraged me to pursue my goals and have provided me with unconditional love and support, what ever my endeavors might be. I am exceedingly grateful for their love and encouragement. My grandfather, Clyde Griffin, has always been a source of inspiration; getting a college degree (Phi Beta Kappa!) during the Great Depression can be nothing less than inspirational! My in-laws, Gary and Sharon Ramsey, are fantastic people and have been very supportive of me from the time I met them.

Finally, there is my dear wife, Holly. Simply put, she is the single greatest person in my life. Holly has provided me with incomparable love and support in the few years I 
have known her. She is a source of inspiration for me and has brought true balance to my

life. One of Holly's most remarkable traits is that she is always cheerful and chooses to see the positive side of things. I love her very much and I look forward to spending many more years with her, exploring what life brings us. 
ABSTRACT

IN MEMORIAM

iv

ACKNOWLEDGEMENTS.

$\mathrm{V}$

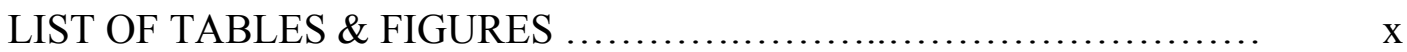

LIST OF ABBREVIATIONS ........................................ xii

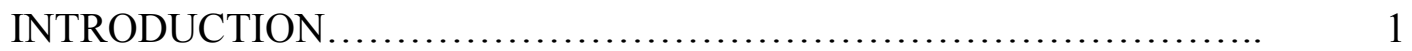

DISSERTATION OBJECTIVES................................... 32

METHODS........................................................ 36

Behavioral Methods

Drug Study Methods

Biochemical Methods

Statistical Methods

RESULTS.

Part A, Parameters of Development

Part B, Preliminary Chronic Mild Stress Experiments

Part C, Acoustic Startle Responding

Part D, Conditioned Fear Assessment

Part E, Signal Transduction

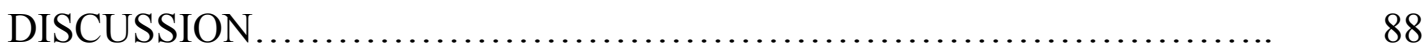

FUTURE DIRECTIONS ...................................... 126

CURRICULUM VITAE.............................................. 128

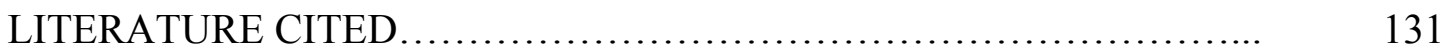




\section{LIST OF TABLES AND FIGURES}

TABLES $\quad$ Page

Table 1. Gestational parameters.................................. 52

Table 2a. Litter size and P1 death rate.............................. 53

Table 2 b. P1 body weight as a function of litter size................... 53

Table 3. Series of behavioral tests................................. 60

Table 4. Adrenal weights....................................... $\quad 62$

Table 5. Latency to peak startle response in the FPS experiments.......... 83

\section{FIGURES}

Figure 1. Acoustic Startle Pathway................................ 20

Figure 2. Freezing Pathway...................................... 26

Figure 3. Fear-Potentiated Startle Methods.......................... 42

Figure 4. Fear-Conditioned Freezing Methods........................ 43

Figure 5. Pre-weanling Body Weights and AG Distances................ 54

Figure 6. Naïve Adolescent and Adult Body Weights and AG Distances... 55

Figure 7. Pre-weanling CRH Content in the BNST, CeA/BLA and PVNH.. 56

Figure 8. Adolescent and Adult CRH Content in the BNST, CeA/BLA, PVNH and DRN.............................. 57

Figure 9. Sucrose intake and body weight during chronic mild stress........ 61

Figure 10. Peak startle responding and latency to peak response with variable decibel exposure...

Figure 11. Peak startle responding in the presence of 8-OH-DPAT and WAY-100635. 
Figure 12. Latency to peak startle response in the presence of 8-OH-DPAT and WAY-100635.................... 68

Figure 13. Percent Difference Scores from several FPS experiments........ 76

Figure 14. FPS peak startle responding after 2 and 4 training days.......... 77

Figure 15. FPS null period responding after 2 and 4 training days............ 78

Figure 16. Behavioral inhibition to context.............................. 79

Figure 17. Behavioral inhibition to an explicit light cue.................... 80

Figure 18. FPS results and difference scores within session................ 81

Figure 19. FPS null period responding ................................ $\quad 82$

Figure 20. $\left[{ }^{3} \mathrm{H}\right] 8-\mathrm{OH}-\mathrm{DPAT}$ binding in tissue homogenates................. 85

Figure 21. cAMP production in tissue homogenates...................... 87 


\section{LIST OF ABBREVIATIONS}

5-HIAA:

5-HT:

5-HTP:

8-OH-DPAT:

ACTH:

AG:

Amygdaloid Nuclei

BLA:

CeA:

Lat:

ANOVA:

ASR:

BNST:

cAMP:

$\mathrm{CON}$ :

CMS:

cpm:

CRE:

CRH:

CRHir:

CRN:

CS:

CSF:

dpm:

DRN:

DSA:

FPS:

$\mathrm{g}$ :

GRE:

HPA:

i.c.v.:

i.p.:

ITI:

$\mathrm{Kg}$ :

L:

LC:

ME:

$\mu$ :

mg:

MRN:

$\mathrm{nM}$ :

NSA:
5-Hydroxyindolacetic Acid

5-Hydroxytryptamine or Serotonin

5-Hydroxy-L-tryptophan

8-hydroxy-2-(di-n-propylamino)tertralin hydrobromide

Adrenocorticotropin Hormone

Ano-genital

Basolateral Nucleus

Central Nucleus

Lateral Nucleus

Analysis of Variance

Acoustic startle responding

Bed Nucleus of the Stria Terminalis

cyclic adenosine monophosphate

not prenatally stressed (note: used as an adjective)

Chronic mild stress

counts per minute

cAMP response element

Corticotropin-Releasing Hormone

Corticotropin-Releasing Hormone immunoreactivity

Cochlear root nucleus

Conditioned stimulus

Cerebrospinal fluid

Decays per minute

Dorsal raphe nucleus

Daytime sucrose assay

Fear-potentiated startle

Gram

Glucocorticoid Response element

Hypothalamic-Pituitary-Adrenal

Intracerebroventricular

Intraperitoneal

Inter-trial interval

Kilogram

Liter

Locus coerulus

Median eminence

micro

milligram

Medial raphe nucleus

nanomolar

nighttime sucrose assay 


\section{List of Abbreviations, continued}

pg:

PAG:

PBN:

PnC:

PS:

PVNH:

RIA:

RM:

S.c.:

SOM:

SPL:

US:

vPAG:

WAY-100635: picogram

Periaqueductal gray

Parabrachial Nucleus

Nucleus reticularis pontis caudalis

Prenatally Stressed (note: this abbreviation is used as an adjective)

Paraventricular nucleus of the hypothalamus

Radioimmunoassay

Repeated measures

Subcutaneous

Somatostatin

Sound pressure level

Unconditioned Stimulus

Ventral periaqueductal gray

(N-[2-[4-(2-methoxyphenyl)-1-piperazinyl]ethyl]-N-(2-

pyridinyl)cyclohexane carboxamide trihydrochloride maleate 


\section{INTRODUCTION}

Early life experiences shape how humans and other animals react to the world around them as adults. Contemporary research demonstrates that the developing brain is quite malleable and, therefore, susceptible to alteration by outside influences. A number of studies describe the detrimental effects of alcohol use, illicit drug use and even therapeutic drug use on normal development of the fetus [e.g.(Hepper, 1995) (Tavares and Silva, 1996) (Heaton et al., 1995) (Lee and Rivier, 1994) (Canger et al., 1999)]. It is also clear that chronic maternal psychological stress during pregnancy disrupts normal development of the fetal brain, creating the potential for behavioral and emotional problems later in life.

\section{The Prenatal Stress Syndrome}

In 1941, Sontag synthesized the available information regarding maternal-fetal interactions and proposed the hypothesis that behavioral disturbances in some children may have their origin during pregnancy (Sontag LW, 1941). Sontag supported his hypothesis with anecdotal evidence. The first true attempt at quantifying this hypothesis did not occur until 1956 with a study by Pasaminick et. al. who demonstrated that stressful complications during pregnancy were associated with behaviorally disturbed children (Pasamanick et al., 1956). One year later, Thompson studied the effects of inescapable stress on pregnant rats (Thompson, 1957). In that study by Thompson, female rats were trained to avoid a footshock in one chamber by escaping through a door to another chamber when a buzzer sounded. Later, during pregnancy the door was closed to prevent escape when the buzzer sounded, although no shock was delivered. The male offspring of those mothers were less active in an open field, had longer latencies to emerge from their home cage and longer latencies to approach food after 24 hours of food deprivation. Another study published 
later that same year, by D.H. Stott, was a retrospective study on stress during human pregnancies (Stott, 1957). Stott found strong correlations between mental retardation and maternal psychological stress during pregnancy. Together, the studies by Pasaminick et. al., Thompson and Stott provided the first quantifiable evidence that psychological trauma during pregnancy was detrimental to the fetus and initiated the study of the prenatal stress syndrome.

Further studies of human pregnancies demonstrated that children of mothers exposed to psychological stressors involving difficult inter-personal relationships were more likely to express behavioral and emotional problems (e.g. excessive crying, clinging, fretfulness, neuroticism) than children with no such background (Stott, 1973) (Ward, 1991) (Stott and Latchford, 1976). Stott also found that children subjected to prenatal stress were more likely to experience developmental delays (late walking or poor walking ability) as well as an increased incidence of physical ailments (eczema, respiratory problems) (Stott, 1973). Other studies demonstrated that the death of the father or the experience of war during pregnancy correlated with behavioral disturbances in children, including schizophrenia (Huttunen and Niskanen, 1978) (Meijer, 1985) (Van Os and Selten, 1998). Together, these studies suggest that prolonged maternal psychological stress during pregnancy increases the likelihood of emotional disturbances or developmental delays in children.

Experiments utilizing non-human primates, stressed during gestation, nicely complement the human studies. Prenatally stressed (PS) Rhesus monkeys demonstrate more clinging behavior and less social contact with their peers under baseline and stressful conditions when compared with non-prenatally stressed (CON) monkeys (Clarke and 
Schneider, 1993). After exposure to novelty, the PS monkeys show less exploratory behavior than CON monkeys, indicating fearfulness (Schneider, 1992). Investigations into motor activity have also revealed that PS monkeys have impaired neuromotor maturity; that is, activities utilizing neuromuscular coordination do not develop as quickly in PS monkeys (Schneider, 1993). Finally, another study examined the hypothalamic-pituitaryadrenal (HPA) axis activity and indicated that adrenocorticotropin hormone (ACTH) levels were greater at all instances measured in PS monkeys than in CON monkeys. This implies that the HPA axis of the PS monkeys is not appropriately regulated and hints at an underlying mechanism for the behavioral abnormalities (Clarke et al., 1994).

Many studies of the prenatal stress syndrome have used rats. Behaviorally speaking, PS rats are less active in open field tests than CON rats [(Suchecki and Palermo, 1991) (Wakshlak and Weinstock, 1990), but see (Poltyrev et al., 1996)]. PS rats also spend less time in the open arms of the elevated plus maze (Poltyrev et al, 1996) (Fride and Weinstock, 1988). Additionally, PS rats demonstrate more behavioral inhibition (i.e. freezing) to acute footshock than non-PS rats (Takahashi et al., 1992a) (Takahashi et al., 1990). Finally, PS rats display increased defensive withdrawal, before and after 2 hours of restraint (Ward et al., 2000). These studies suggest that PS rats are more fearful in novel or stressful environments. Although it is difficult to directly extrapolate these findings to the human condition, the behavioral disturbances seen in prenatally stressed children may be signs of fear related to novel or stressful situations.

A hallmark feature of prenatal stress in rats is HPA axis dysregulation. A number of investigators have reported that PS rats have greater plasma corticosterone and $\mathrm{ACTH}$ levels than CON rats following a stressful intervention, including exposure to an open field 
(Fride et al., 1986) (Peters, 1986a). Furthermore, Peters reported elevated plasma

corticosterone and $\mathrm{ACTH}$ following a subcutaneous (s.c.) $0.9 \%$ saline injection in adult PS rats (Peters, 1982). In addition to this phenomenon occurring in adult rats, Takahashi has shown this to be true for young, pre-weanling PS rats following a footshock (Takahashi and Kalin, 1991). Elevated corticosterone and ACTH after a stressful intervention may be due to decreased numbers of hippocampal glucocorticoid receptors (type I and II) in PS rats, which are integral parts of the negative feedback loop of the HPA axis (Barbazanges et al., 1996) (Koehl et al., 1999) (Gilman et al., 1990). Regardless of these observations, basal levels of corticosterone and ACTH do not appear to be drastically different between male PS and CON rats. Koehl et. al., using chronic indwelling venous catheters, reported no differences in daily basal corticosterone levels throughout most of the day, although they did find that the daily peak response was somewhat higher and occurred sooner in male PS rats than in CON rats (Koehl et al, 1999).

Unfortunately, studies exploring HPA axis regulation in PS humans have not been published and studies in PS non-human primates are rare. In fact, only two studies could be found that have been published regarding HPA axis activity in PS non-human primates. The first was alluded to earlier and showed differences in levels of ACTH (Clarke et al, 1994). The other study, by Reyes and Coe, looked at HPA axis response to interleukin-6 challenge and did not see differences between PS and CON monkeys; however, the prenatal stressor was quite mild and involved a single exposure to noise during the middle of the pregnancy (Reyes and Coe, 1997). Clearly, more studies are needed in humans and non-human primates to determine if the effects found in rodents are pertinent to the human condition. 
Although most research about prenatal stress has focused on male subjects, particularly in rodents, it should be noted that prenatal stress affects both genders but not necessarily in the same way. In most studies regarding prenatal stress in human and nonhuman primates, distinctions about gender effects are left unstated, leaving the reader to assume that prenatal stress affects males and females equally [e.g. (Clarke et al, 1994) (Clarke and Schneider, 1993) (Ward, 1991) (Stott, 1973)]. Only one study could be found that compared PS and CON monkeys in terms of gender and prenatal condition, but no differences were detected in this regard (Schneider, 1992). The lack of gender effects in this study may be related to the small number of subjects in the PS and CON groups.

On the other hand, in rodent models, investigators have reported clear differences with regard to the effects of prenatal stress on males and females. For example, PS female rats have higher basal plasma corticosterone levels during the nadir in the corticosterone circadian rhythm than CON female rats; whereas, PS and CON male rats do not differ in this respect (Koehl et al, 1999) (Szuran et al., 2000). Behaviors are also differentially affected by prenatal stress with regard to gender. Szuran et. al. reported that cold water escape latencies for PS females were faster at $10^{\circ} \mathrm{C}$ than either PS males, CON males or CON females which were all essentially equal in escape latency (Szuran et al, 2000). Additionally, Fride and Weinstock noted that PS female rats spend more time on the open arms of the plus-maze than PS males, indicating that the PS females are less fearful than their male counterparts (Fride and Weinstock, 1988).

As with the paucity of studies on HPA axis regulation in primate species, more work needs to be done with regard to gender effects and prenatal stress to determine if the findings with gender effects in rodents apply to the human condition. Given the gender 
differences found in rodents, it seems reasonable to speculate that if the results of human and non-human primates were stratified according to gender, some instances where differences were not detected originally may actually yield differences. For example, Clarke et. al. in their studies with Rhesus monkeys and HPA axis activity only found effects with ACTH and not cortisol (Clarke et al, 1994). However, they did not collect blood over a 24 hour period and did not sort the results by gender (the cohorts were composed of males and females). The results from rodent studies using catheters suggest that the timing of blood collection and the gender of the subject are important variables to control with regard to prenatal stress and HPA axis regulation.

A major tenet of the prenatal stress syndrome is that the stressor is applied chronically for some or all of the gestational period. Furthermore, the mother is not given a chance to adapt or cope with the stressor. In fact, the original experiment in rats performed by Thompson included this as part of the method of stressing (Thompson, 1957). In discussing their studies on human pregnancies, D.H. Stott and A.J. Ward both point out that the common denominator with the PS syndrome was that the mothers were in situations with which they could not easily cope or escape (Stott, 1973) (Ward, 1991). In accordance with this observation, the non-human primate and rodent studies cited above all contained a chronic stressor applied for large portions or all of the gestational period. Moreover, several studies cited above examined single stressful events during pregnancy and failed to replicate the findings from the chronically stressed pregnancies [e.g. see (Stott, 1973) (Schneider, 1993)].

What, then, causes the prenatal stress syndrome? The leading candidate is excessive circulating maternal glucocorticoids resulting from the applied stressor, which is 
corticosterone for rats and cortisol for humans [see reviews, (Ward, 1984) (Weinstock, 1997)]. Glucocorticoids are relatively lipophilic, enabling them to cross the placental barrier and gain access to the fetal compartment (Zarrow et al., 1970). Additionally, the stressors employed in many studies (e.g. footshock, saline injection, restraint) are known to elevate plasma glucocorticoids well above baseline levels. Indeed, several investigators have reported increased maternal as well as fetal plasma glucocorticoids after the stressful intervention with the mother (Ward and Weisz, 1984) (Williams et al., 1999) (Barbazanges et al, 1996) (Skinner and Birkle, unpublished observations). This seems to be in spite of the presence of 11- $\beta$-hydroxysteroid dehydrogenase, an enzyme expressed in the placenta and thought to protect the fetus from the natural rise in plasma glucocorticoids associated with pregnancy (Yang, 2000). Interestingly, blockade of this enzyme with carbenoxolone during an otherwise normal pregnancy in rats results in offspring which are behaviorally very similar to PS rats, presumably because the normal levels of maternal glucocorticoids gained access to the fetal compartment (Welberg et al., 2000).

Glucocorticoids, when bound to their intracellular receptors and translocated to the nucleus, function as transcription factors and will ultimately affect levels of a diverse number of proteins (Meyer, 1985) (Gilman et al, 1990). Glucocorticoids decrease or repress the production of gene products such as corticotropin-releasing hormone $(\mathrm{CRH})$, glucocorticoid receptors and serotonergic 5- $\mathrm{HT}_{1 \mathrm{~A}}$ receptors, all of which are of interest to the study of the prenatal stress syndrome (Malkoski et al., 1997) (Biegon et al., 1985) (Sze et al., 1976) (Sapolsky and McEwen, 1985) (Zhong and Ciaranello, 1995) (Meijer et al., 2000). 
Alternatively, glucocorticoids can also increase levels of some proteins such as tryptophan hydroxylase, tyrosine hydroxylase and even CRH under the right circumstances (Azmitia and McEwen, 1974) (Meyer, 1985) (Tanimura and Watts, 1998). To further complicate matters, the effects of glucocorticoids on transcription vary by brain region. For example, adrenalectomy leads to increases in numbers of $5-\mathrm{HT}_{1 \mathrm{~A}}$ receptors in the hippocampus but leaves numbers of $5-\mathrm{HT}_{1 \mathrm{~A}}$ receptors unchanged in the dorsal raphe nucleus (DRN), indicating that different transcriptional regulatory elements control expression in those brain regions (Meijer et al, 2000) (Laaris et al., 1995).

Therefore, chronically exposing the fetus to excessive levels of maternal glucocorticoids may lead to modulation of RNA transcript levels and subsequent protein expression early in life and may be the ultimate culprit behind the prenatal stress syndrome. Furthermore, PS animals continue to face the problem of excessive glucocorticoid exposure during stressful events as they grow older due to deficits in the HPA axis negative feedback loop.

The preceding observations and comments regarding prenatal stress naturally lead to speculations about whether it serves an adaptive or a maladaptive function for the offspring. Certainly, when taken at face value, the results of the human and the non-human primate studies suggest that prenatal stress is a maladaptive phenomenon. PS offspring that are behaviorally disruptive or that demonstrate excessive clinging (and perhaps fear?) would find it difficult to benefit from the social support that is so important for gregarious societies like those of primates. Additionally, the HPA axis regulation problem described in the previous paragraphs would suggest that multiple exposures to stressful events would also be detrimental in PS offspring. Nevertheless, if many of the behavioral sequelae that 
characterize prenatal stress are indicative of enhanced fear, then perhaps there are situations where prenatal stress also serves an adaptive function since fear is such an important, basic emotion for the preservation of life. For example, in the case of PS rats, which have been shown to demonstrate enhanced behavioral inhibition, remaining motionless for extended periods of time in the face of a threat may help them evade detection by a predator.

Additionally, PS rats are also known to display more defensive withdrawal behavior, which may keep them from being spotted by a predator in the first place. Ultimately, whether prenatal stress is adaptive or maladaptive may only be decided by the circumstances in which the animal is involved and how long those circumstances last.

\section{The CRHergic Neurotransmitter System}

Vale and colleagues discovered the molecule now known as corticotropin-releasing hormone (CRH) in 1981 (Vale et al., 1981). When released into the portal veins of the median eminence (ME) by the parvocellular neurons of the paraventricular nucleus of the hypothalamus (PVNH), $\mathrm{CRH}$ is a potent secretagogue of $\mathrm{ACTH}$ from the cells of the anterior pituitary [for a brief review, (Fisher, 1989)]. Thus, CRH plays the role of initiator for the classic HPA axis response to stress. It is probable that increased CRH release from the ME could account for the hyper-responsive HPA axis described in PS rats. Only one study has examined this possibility, and although there was a larger amount of $\mathrm{CRH}$ found in the ME of PS rats, statistical differences were not detected, and the investigators were looking at content not release (Smythe et al., 1996).

It is also appreciated that $\mathrm{CRH}$ is a neurotransmitter in other areas of brain and plays an important role in modulating behavior, particularly fearful behaviors [for reviews, 
see (Owens and Nemeroff, 1991) (Dunn and Berridge, 1990)]. In fact, if CRH is administered intracerebroventricularly (i.c.v.), rats display behaviors indicative of increased fearfulness such as increased defensive withdrawal, increased stress-induced freezing, and elevated startle responses (Ward et al., 1998) (Kalin et al., 1988) (Liang et al., 1992) (Sherman and Kalin, 1988). Interestingly, i.c.v. CRH is also associated with increased grooming in rats, implying that under some circumstances grooming may be a sign of anxiety in rodents [reported in (Owens and Nemeroff, 1991)]. Importantly, many of these effects are blocked by CRH antagonists also injected i.c.v.(Liang et al, 1992) (Ward et al, 2000) (Kalin et al, 1988). Finally, a CRH over-expressing mouse displays signs of anxiety (e.g. decreased open arm time); although, as predicted, it does suffer from a syndrome very much like Cushing's disease (Stenzel-Poore et al., 1996).

Endogenous, extrahypothalamic CRH containing neurons are found in brain regions such as the central nucleus of the amygdala $(\mathrm{CeA})$, bed nucleus of the stria terminalis (BNST), dorsal raphe nucleus (DRN), parabrachial nucleus (PBN) and the locus ceruleus (LC) (Palkovits et al., 1985) (Skofitsch and Jacobowitz, 1985) (Swanson et al., 1983) (Cummings et al., 1983). CRH receptors are found in these regions as well (De Souza, 1987) (Potter et al., 1994) (Cummings et al, 1983). These brain regions are associated with fear responses in a variety of experimental paradigms and CRH plays a role in those fear responses. For example, $\mathrm{CRH}$ micro-injected into the amygdala decreases interaction time in the social interaction test and will reduce exploratory behavior (Sajdyk et al., 1999) (Liang and Lee, 1988). Ablation of the BNST abolishes the elevation of startle responding seen when CRH is administered i.c.v., implying that the CRHergic system in this nucleus is responsible for elevated startle with this route of administration (Lee and Davis, 1997). 
Lastly, intra-amygdalar infusions of $\mathrm{CRH}$ increase aggressive behavior (number of fighting bouts) between rats (Elkabir et al., 1990).

Of course, $\mathrm{CRH}$ accomplishes these actions through its receptors found throughout the brain (De Souza, 1987) (Potter et al, 1994) (Steckler and Holsboer, 1999). There are at least two $\mathrm{CRH}$ receptor subtypes that have been discovered, $\mathrm{CRH}_{1}$ and $\mathrm{CRH}_{2}$ (Lovenberg et al., 1995b) (Chalmers et al., 1995). Additionally, the $\mathrm{CRH}_{2}$ receptor is known to have two major splice variants, $\mathrm{CRH}_{2 \alpha}$ and $\mathrm{CRH}_{2 \beta}$ which have been differentiated by pharmacological and molecular biological techniques (Lovenberg et al., 1995a) (DeSouza, 1995). Two other shorter splice variants of the $\mathrm{CRH}_{2}$ receptor have been discovered recently and classified based on sequence homology and in vitro pharmacological studies. The $\mathrm{CRH}_{2 \alpha-\text { tr }}$ receptor was cloned from rat amygdala while the $\mathrm{CRH}_{2 \gamma}$ receptor was found to be expressed in several limbic regions in the human brain (Miyata et al., 1999) (Kostich et al., 1998). It remains to be seen what role the $\mathrm{CRH}_{2 \alpha-\text {-r }}$ and $\mathrm{CRH}_{2 \gamma}$ receptors have in behavior.

$\mathrm{CRH}_{1}$ and $\mathrm{CRH}_{2 \alpha}$ receptors are differentially located throughout the brain suggesting different roles in behavior. For example, the density of the $\mathrm{CRH}_{1}$ receptors is quite high in areas of the brain dealing with the stress response such as the amygdala, hippocampus, BNST, pituitary and olfactory bulbs (DeSouza, 1995). The $\mathrm{CRH}_{1}$ receptor is thought to be the major receptor of the CRHergic system that mediates fear and anxietylike behaviors and, of course, causes release of ACTH in the classic HPA axis cascade (Steckler and Holsboer, 1999). The $\mathrm{CRH}_{2 \alpha}$ receptor is found in high quantities in the lateral septum, PVNH and ventromedial nucleus of the hypothalamus (Chalmers et al, 1995) (Lovenberg et al, 1995a). A review of the literature suggests a role for $\mathrm{CRH}_{2 \alpha}$ 
receptors in reducing food intake, but other roles similar to those of the $\mathrm{CRH}_{1}$ receptor cannot be ruled out (Eghbal-Ahmadi et al., 1999) (Steckler and Holsboer, 1999). On the other hand, the $\mathrm{CRH}_{2 \beta}$ receptor is largely found in non-neuronal tissue, particularly cardiac muscle, skeletal muscle, cerebral arterioles and choroid plexus (Lovenberg et al, 1995a). The distribution of the $\mathrm{CRH}_{2 \beta}$ receptor makes it unlikely that these receptors play a direct role in generating behaviors.

The CRH receptors are members of the G-protein coupled, seven transmembranespanning receptor superfamily of proteins (Steckler and Holsboer, 1999). Primary cell culture experiments and tissue homogenate experiments indicate that the $\mathrm{CRH}_{1}$ and $\mathrm{CRH}_{2 \alpha}$ receptors are positively coupled to cAMP production (Lutz-Bucher et al., 1991) (Battaglia et al., 1987). The reported $\mathrm{EC}_{50}$ measurements in tissue homogenates of $\mathrm{CRH}$ at the $\mathrm{CRH}_{1}$ receptor for cAMP production is $36 \mathrm{nM}$ (Battaglia et al, 1987). The $\mathrm{EC}_{50}$ value in cellular expression systems of $\mathrm{CRH}$ for cAMP production at the $\mathrm{CRH}_{1}$ receptor has been reported to be in the 3 to $4 \mathrm{nM}$ range (Chen et al., 1993) (Lovenberg et al, 1995b). The $\mathrm{EC}_{50}$ value for cAMP production of $\mathrm{CRH}$ at the $\mathrm{CRH}_{2 \alpha}$ receptor in cellular expression systems is a little higher at $20 \mathrm{nM}$ (Lovenberg et al, 1995b).

In that same system used by Lovenberg et. al., another endogenous peptide related to $\mathrm{CRH}$, urocortin, produced an $\mathrm{EC}_{50}$ value of $2 \mathrm{nM}$ for cAMP production, suggesting that CRH may not be the endogenous ligand for the $\mathrm{CRH}_{2 \alpha}$ receptor (Lovenberg et al, 1995b) (Koob and Heinrichs, 1999). This idea is also supported by the observation that c-fos induction is stronger and correlates better with $\mathrm{CRH}_{2 \alpha}$ distribution following i.c.v. urocortin administration than after i.c.v. CRH administration (Bittencourt and Sawchenko, 
2000). Certainly, more work needs to be done before the implications of these findings are fully understood.

The CRHergic system develops late during gestation in the rat. CRH mRNA is detectable by gestational day 17 in rat brain (Baram and Schultz, 1992). One of the CRH receptors $\left(\mathrm{CRH}_{2}\right)$ is detectable a little earlier at gestational day 16 (Eghbal-Ahmadi et al., 1998). Consequently, it has been shown that prenatal stress alters the CRHergic system. Cratty et. al. discovered that amygdala minces from PS rats, when stimulated by high potassium buffers, release more CRH than minces from CON rats (Cratty et al., 1995). That same study also showed that CRH content was increased in block dissected tissue taken from PS rats containing the amygdala. Another study demonstrated increased CRH receptor binding in the amygdala of PS rats (Ward et al, 2000). Finally, i.c.v. injected CRH antagonists block the increase in defensive withdrawal seen in PS rats (Ward et al, 2000). Thus, the developing CRHergic system is vulnerable to manipulation by outside influences during the latter part of gestation in the rat. Furthermore, these observations suggest that an up-regulated CRHergic system may be at least partially responsible for the behavioral differences between PS and CON rats.

\section{The Serotonergic Neurotransmitter System}

In recent years, the role of serotonergic system in psychiatric disorders has become evident, particularly with regard to anxiety and depression. Interest in this system is spurred on by a variety of drugs used in clinical practice that target elements of the serotonergic system, e.g. fluoxetine and buspirone, that are efficacious in treating various psychiatric disorders. A plethora of reviews on the serotonergic system have been 
published and, consequently, theories abound regarding the serotonergic system's involvement in these afflictions and behavior in general (Graeff et al., 1996) (Chaouloff, 1993) (Chopin and Briley, 1987) (Handley and McBlane, 1993). One thing is quite clear, the involvement of the serotonergic system in any behavior is complicated because of the diverse nature of the system.

The majority of serotonergic neurons, discriminated by tryptophan hydroxylase labeling, arise in the DRN and medial raphe nucleus (MRN), although there are serotonergic neurons found arising in the raphe magnus and raphe obscurus as well (Tork, 1990). The DRN is usually considered to supply the majority of serotonergic innervation to the brain (Tork, 1990). The neurons of the DRN project to many locations in the brain, particularly the forebrain, but there are projections to areas of the brainstem as well. Targets of the DRN include: the hippocampus, amygdala, cortex, MRN, PVNH, reticularis pontis caudalis (RPC) and periaqueductal gray (PAG) (Tork, 1990) (Vertes and Kocsis, 1994).

Serotonin exerts its effects through a huge variety of receptors. At the present time there are 7 known families of serotonin receptors with several families having a number of subtypes differentially located throughout the brain (Hoyer et al., 1994) (Radja et al., 1991). These receptors belong to the G-protein coupled, seven transmembrane-spanning family of receptor proteins and initiate a variety of signal transduction systems including cAMP production, phospholipid hydrolysis and ion channel activation (Pauwels, 2000). One of the best characterized subtypes, the $5-\mathrm{HT}_{1 \mathrm{~A}}$ receptor, is found in a number of brain regions associated with limbic functions and is involved in emotionally driven behaviors. 
The $5-\mathrm{HT}_{1 \mathrm{~A}}$ receptor is found in discrete regions throughout the brain as demonstrated by autoradiographic techniques using ${ }^{3} \mathrm{H}-8-\mathrm{OH}-\mathrm{DPAT}$, a selective agonist of this receptor (Palacios et al., 1990). Particularly dense populations of 5-HT $\mathrm{HA}_{1 \mathrm{~A}}$ receptors are found in the dentate gyrus of the hippocampus and the DRN (Hoyer et al., 1986). Other locations supporting populations of 5- $\mathrm{HT}_{1 \mathrm{~A}}$ receptors are the amygdala, entorhinal cortex, lateral septum, PVNH and striatum (Hall et al., 1985) (Radja et al, 1991).

The use of the serotonergic neurotoxin, 5,7-dihydroxytryptamine, has shown that 5$\mathrm{HT}_{1 \mathrm{~A}}$ receptors are found pre-synaptically and post-synaptically (Verge et al., 1986). Receptors found in the DRN are somatodendritic autoreceptors, i.e. found on the cell bodies and dendrites (Verge et al, 1986). The available evidence supports the idea that these somatodendritic autoreceptors receptors are coupled to inwardly rectifying potassium channels and, when activated, promote hyperpolarization of the DRN neurons to inhibit neuronal firing (Mundey et al., 1996) (Forster et al., 1995). Conversely, 5- $\mathrm{HT}_{1 \mathrm{~A}}$ receptors in other brain regions are post-synaptic relative to the DRN projections. These postsynaptic receptors are usually found negatively coupled to cAMP production with reported $\mathrm{IC}_{50}$ values ranging from 1 to $18 \mathrm{nM}$ in tissue homogenates (De Vivo and Maayani, 1986) (Mork and Geisler, 1990) (Koyama et al., 1999). However, there are reports of post-synaptic 5-HT $\mathrm{HA}_{1 \mathrm{~A}}$ receptors being coupled to potassium channels in the hippocampus having $\mathrm{EC}_{50}$ values for 5-HT (not 8-OH-DPAT) of about $5 \mathrm{nM}$ (Okuhara and Beck, 1998) (De Vivo and Maayani, 1986).

Two pharmacological tools permit the study of 5- $\mathrm{HT}_{1 \mathrm{~A}}$ receptor involvement in behavior. Systemic treatment with low doses $(<1 \mathrm{mg} / \mathrm{kg})$ of the $5-\mathrm{HT}_{1 \mathrm{~A}}$ agonist, $8-\mathrm{OH}-$ DPAT produces anxiolytic responses in the elevated plus maze, the social interaction test 
and shock-induced vocalization (Collinson and Dawson, 1997) (Picazo et al., 1995) (Remy et al., 1996). Conversely, at higher doses, systemic 8-OH-DPAT elevates acoustic startle responding, which is considered an anxiogenic response (Svensson, 1985). Davis and colleagues have reported that systemically administered 8-OH-DPAT does not block fearpotentiated startle responses, but does not increase fear-potentiation either (Davis et al., 1999). Low doses ( $<1 \mathrm{mg} / \mathrm{kg}$ ) of 8-OH-DPAT are also anxiogenic in the defensive withdrawal test (DeBastiani and Birkle, unpublished observations). The second pharmacological tool is the antagonist, WAY-100635 (Gozlan et al., 1995). Treatment with WAY-100635 alone does not produce signs of the serotonin syndrome (Fletcher et al., 1996). WAY-100635 given in combination with 8-OH-DPAT will antagonize behavioral responses to 8-OH-DPAT as well as prevent decreases in DRN neuronal firing rates caused by 8-OH-DPAT (File et al., 1996) (Fletcher et al, 1996) (Remy et al, 1996).

Micro-injections of 8-OH-DPAT into discrete brain regions such as the DRN and MRN are also anxiolytic in the ultrasonic vocalization test, social interaction test, the elevated plus maze and tend to reduce maternal aggression and these anxiolytic actions are blocked by WAY-100635 (Remy et al, 1996) (Picazo et al, 1995) (File et al, 1996) (De Almeida and Lucion, 1997). Conversely, injections of 8-OH-DPAT into the dorsal hippocampus are anxiogenic in the social interaction test as well as in the elevated-plus maze when rats are not naïve to the plus maze (File et al, 1996). 8-OH-DPAT injected into the medial septal area tends to increase maternal aggression, but the effect is not very strong (De Almeida and Lucion, 1997).

As a final point, two different laboratories have generated 5- $\mathrm{HT}_{1 \mathrm{~A}}$ knock-out mice from different genetic backgrounds that display many signs of anxiety such as decreased 
locomotion in an open-field and decreased time in open-arms of the elevated plus maze (Ramboz et al., 1998) (Parks et al., 1998). On balance, then, it would appear that activation of $5-\mathrm{HT}_{1 \mathrm{~A}}$ receptors produces an anxiolytic response, particularly the presynaptic receptors. However, this is not always the case, considering the effects of systemic 8-OH-DPAT on acoustic startle responding, defensive withdrawal test as well as the effects of intrahippocampal injections of 8-OH-DPAT on the elevated plus maze. Ultimately, these discrepancies may be explained by determining which brain regions containing $5-\mathrm{HT}_{1 \mathrm{~A}}$ receptors are important (if at all) to a given behavioral response.

Like the CRHergic system, the serotonergic system is vulnerable to the effects of gestational stress due to its course of development. Serotonergic cell bodies, discriminated by tryptophan hydroxylase labeling, are detectable in the brainstem of fetal rats by gestational day 12 (Aitken and Tork, 1988). Accordingly, Peters reported that prenatal stress affects elements of the serotonergic system in a series of studies published in the mid 1980's. Peters found that, upon administration of several doses of 5-hydroxy-1-trytophan (5-HTP), PS rats displayed more "wet dog shakes" (a sign of the serotonin syndrome) than the CON rats at all the doses, except the highest dose (Peters, 1986a). In the same study, measurements of serotonin content from the hypothalamus showed that 5hydroxytryptamine (5-HT) and 5-hydroxyindolacetic acid (5-HIAA) content were decreased in PS pre-weanling rats compared to control, but after weaning the content of 5HT and 5-HIAA in the hypothalamus actually increased in the PS rats compared to control (Peters, 1986a). Conversely, Hayashi et. al. found that 5-HT was decreased in the rat hippocampus at 35 days of age (Hayashi et al., 1998). Peters also discovered that $\left[{ }^{3} \mathrm{H}\right]-5$ HT binding in adult PS rats was increased in the cortex and hippocampus (Peters, 1986a). 
Lastly, Peters compared the effects of performing his gestational stressor (once daily saline injection) at various times during pregnancy with stressing for the entire pregnancy (Peters, 1989). He found that stressing the rats during the last week of the three week gestational period replicated findings from studies where the stressing was conducted for the entire pregnancy (Peters, 1989). This observation fits nicely with the ontogeny of the serotonergic and CRHergic systems and the prenatal stress syndrome.

\section{The Acoustic Startle Response (ASR)}

The ASR is a reflexive contraction of skeletal muscle in response to a sudden, loud noise. The response can be elicited in rats, mice, humans, non-human primates and many other mammalian species. The response itself has a non-zero baseline and can be modulated up or down depending on the emotional state of the animal. The ASR has been studied for quite sometime and there are two excellent reviews on this subject (Koch, 2000) (Davis, 1984).

Two behavioral paradigms demonstrate modulation of acoustic startle responding. The least studied phenomenon is pleasure-attenuated startle. Rats trained to associate a conditioned stimulus (CS) such as a light with a food reward display decreased ASR in the presence of the CS alone (Bechara et al., 1999). This effect appears to be modulated by the nucleus accumbens since ablation of that nucleus abolishes the response to the CS (Koch et al., 1996). Conversely, Brown and colleagues first reported in 1951 that an aversive CSunconditioned stimulus (US) pairing produces an increase in ASR in the presence of the CS alone (Brown et al., 1951). Since that time, many studies have followed describing the phenomenon of fear-potentiated startle [for reviews, (Davis, 1986) (Davis et al., 1987)]. 
These observations, coupled with similar observations in humans, have led to the idea that startle is increased in states of fear and decreased in non-fearful states (Morgan, III et al., 1995) (Grillon et al., 1997).

The neuroanatomical circuitry underlying the basic ASR is rather simple, consisting of an multisynaptic pathway from the Organ of Corti in the ear to motor neurons in the spinal cord (Figure 1) (Koch, 2000). So, like spinally mediated pain withdrawal reflexes, there is a short latency between stimulus and response. Response latencies measured in the human ocular muscles occur on the order of 20 milliseconds while responses recorded at the hind leg of the rat can be as short as 8 milliseconds [reported in (Davis, 1984)]. The short response times support the notion of only a few primary synapses in the primary ASR pathway.

Upward or downward modulation of the ASR occurs by neuronal projections from various higher brain centers, notably the limbic system, to obligatory synapses in the startle pathway (Figure 1). The synapses of critical importance are found in the cochlear root nucleus (CRN), RPC and at the spinal motor neurons (Davis et al., 1982). Of these synapses, the RPC plays a particularly important role in modulating the startle response. The RPC has been identified as an obligatory synapse in the acoustic startle response by lesion as well as stimulation studies (Lee et al., 1996). (Davis et al, 1982) (Leitner et al., 1980). The RPC appears to be involved in other types of startle as well since lesions of the RPC also block shock-induced startle (Leitner et al, 1980).

The CRHergic system positively modulates startle. CRH injected into the ventricles produces a long-lasting elevation in startle responding. Lesion studies suggest 


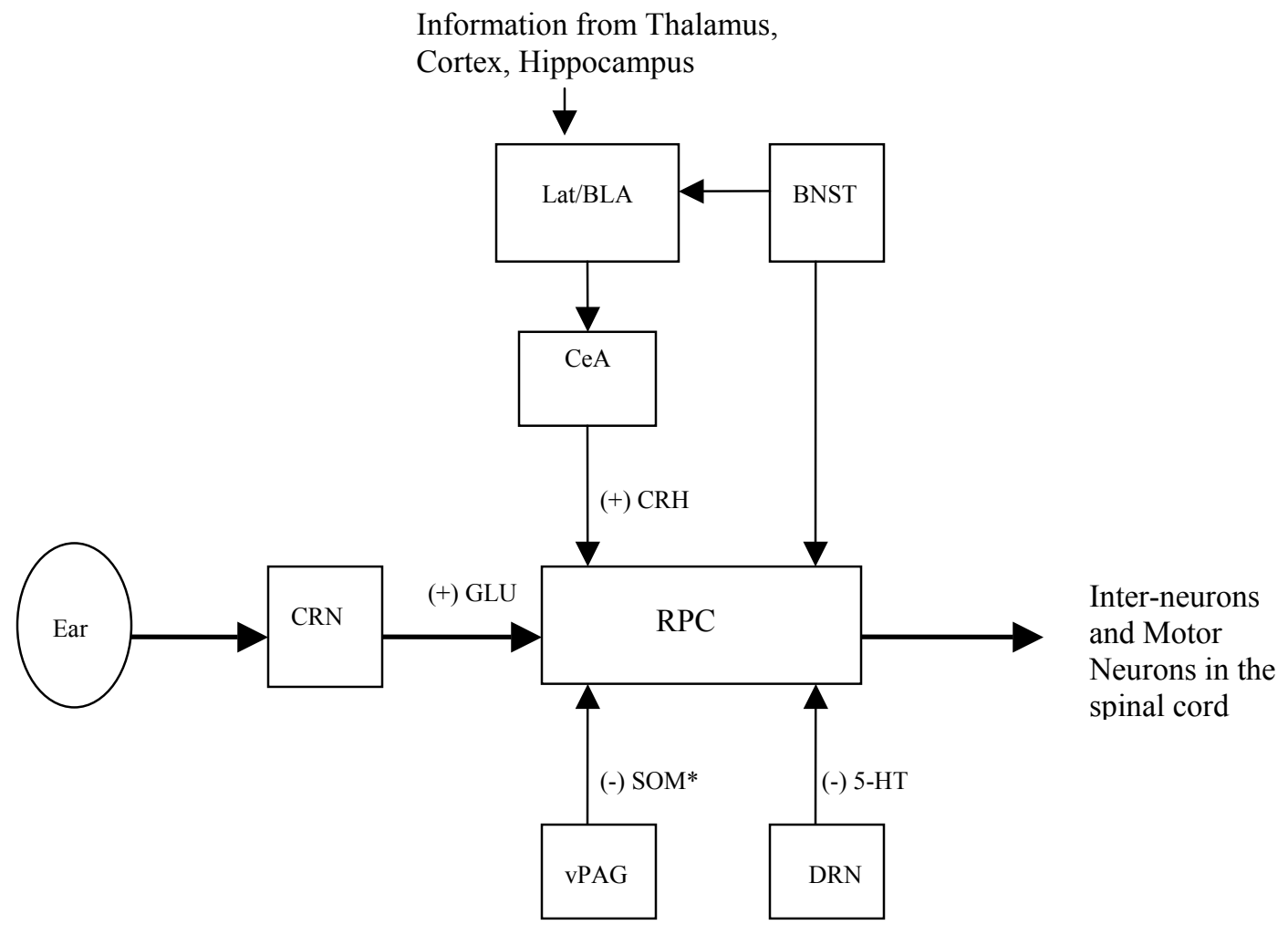

Figure 1. A simplified diagram describing the acoustic startle pathway and its modulation. The primary reflex arc follows the bolded, horizontal arrows. The firing of the motor neurons causes muscular contractions that are the response to sudden, loud acoustic stimuli. Modulatory pathways are known to influence the reflex arc at the level of the RPC. The non-bolded arrows demonstrate known anatomical projections to the RPC and the neurotransmitters involved, where they are known or suspected. $(+)$ indicates a positive effect and (-) indicates a negative effect on startle responding. *This negative affect has only been shown in fear-potentiated startle and does not seem to affect baseline startle responses. (Abbreviations: CRN, Cochlear Root Nucleus; Lat/BLA, Lateral and Basolateral Nucleus of the Amygdala; BNST, Bed Nucleus of the Stria Terminalis; RPC, Reticularis Pontis Caudalis; vPAG, ventral Periaqueductal Gray; DRN, Dorsal Raphe Nucleus; GLU, Glutamate; CRH, Corticotropin Releasing Hormone; 5-HT, Serotonin; SOM, Somatostatin) 
that this effect is mediated by the BNST, a source of CRHergic cell bodies and projections to the RPC (Lee and Davis, 1997). CRH injected into the RPC also increases startle responding (Birnbaum and Davis, 1998) (Fendt et al., 1997). Ablation of the CeA, a rich source of CRHergic cell bodies, does not change startle responding but does eliminate fearpotentiated startle (Campeau and Davis, 1995). In support of this observation, there are anatomical projections to the RPC from the CeA (Rosen et al., 1991). This evidence does not suggest CRHergic tone is important for generating ASR itself, but that it is important for increasing ASR in fearful states.

In contrast to the CRHergic system, the serotonergic system negatively modulates startle. Lesions of the DRN and MRN, thereby removing their influence, elevate startle responding (Davis and Sheard, 1974). Curiously, systemically administered 8-OH-DPAT elevates ASR (Svensson, 1985). This affect of 8-OH-DPAT may be mediated by activation of 5- $\mathrm{HT}_{1 \mathrm{~A}}$ somatodendritic receptors, an action known to depress neuronal activity of these serotonergic neurons (Mundey et al, 1996) (Fletcher et al, 1996). Finally, direct projections from the DRN to the RPC have been demonstrated, providing an anatomical pathway for DRN involvement in the ASR (Vertes and Kocsis, 1994). Together, this evidence suggests an inhibitory influence on the startle response by the serotonergic system arising in the raphe nuclei.

The ASR serves as a useful behavioral index for several reasons. First, the response itself can be elicited in a wide variety of species, including humans, making it possible to test hypotheses across a number of species. Secondly, the acoustic startle response is a non-zero response that can be modulated up or down by conditioning experiences that humans associate with positive or negative emotions. Thus, there is some insight into the 
functional consequences of startle modulation. Lastly, when utilizing a conditioning paradigm, the ASR can be measured in the presence and absence of the CS. This allows the observer to determine if the experimental manipulation affected the acoustic startle response itself.

Does the startle response serve a useful purpose for animals? Acoustic startle responding has been observed in a wide variety of mammalian species. The list includes humans, rats, mice, monkeys, cats, guinea pigs and many others [(Landis and Hunt, 1939)reported in (Davis, 1984)]. Additionally, other types of startle exist such as tactile startle (Engelmann et al., 1996). In general, startle responding is considered to be part of the "Active Defense" system for animals which allows animals to actively engage and monitor threats to their well-being (Handley, 1995).

Time lapse photography of humans and rats show that contraction of flexor muscles is the primary response to startling stimuli, although other muscle groups do get involved (Davis, 1984). This leads to a very characteristic posture for both humans and rats. The shoulders are lifted up towards the ears and the arms are drawn towards the midline of the body. This posture may serve to protect the animal from an impending blow or impact that follows the stimulus. Additionally, some authors have proposed that the characteristic posture "resets" the musculature to allow a quick escape or offensive reaction, which might be particularly important for rodents (Pilz and Schnitzler, 1996). Finally, human subjects report that the experience of startle responding is highly aversive (Handley, 1995). These observations suggest that the startle responding serves to arouse the animal as well as to reset the animal's body posture to protect the animal and prepare the animal for an escape or offensive reaction. 
Only one study has been published so far regarding prenatal stress and ASR (White and Birkle, 2001). In this study, baseline startle responding was largely unaffected by prenatal stress, although the PS rats had slightly elevated startle responses to the first few trials of the test when compared to the $\mathrm{CON}$ rats. Interestingly, the PS rats were less responsive to the anxiogenic effects of yohimbine at $5 \mathrm{mg} / \mathrm{kg}$ when compared to the CON rats (i.e. PS startle responses were not elevated to the same degree as the CON responses). This difference does not seem to be due to a lack of sensitivity to $\alpha_{2}$ adrenoceptor modulation, since a selective drug $(\mathrm{RS} 79982 \mathrm{HCl})$ for that receptor caused the same changes in startle responding between the two groups. Yohimbine is capable of interacting with a wide variety of receptor types, including 5- $\mathrm{HT}_{1 \mathrm{~A}}$ receptors (Arthur et al., 1993) (Winter and Rabin, 1992). Furthermore, 5-HT $\mathrm{HA}_{1 \mathrm{~A}}$ activation increases startle. This raises the possibility that PS rats are less sensitive to $5-\mathrm{HT}_{1 \mathrm{~A}}$ modulation and, therefore, do not demonstrate increased ASR at high doses of yohimbine.

To date, the phenomenon of fear-potentiated startle has not been investigated in the prenatal stress syndrome. However, based on what is known about the behavioral nature of the prenatal stress syndrome, PS rats would be expected to demonstrate larger fearpotentiated responses in this paradigm.

\section{Behavioral Inhibition}

Behavioral inhibition, more commonly known as freezing, is thought to be a defensive behavior that has evolved to protect small animals from being detected by predators (Fanselow, 1991) (Fendt and Fanselow, 1999). Freezing in rats has been recognized and formally studied as an index of fear for many years dating back to 1899 
with detailed observations reported by Small [reported in (Fanselow, 1991)]. However, predatory animals, a group which includes humans, are capable of freezing as well. Although, in the case of predatory animals, the freezing response likely serves to help orient the animal to possible prey (but it can be protective since the predator can sometimes be the prey!) (Reese et al., 1984).

For a rat, freezing behavior describes a rat that, in the face of a threat, ceases all movement except that required to breathe. This can be a long-lasting response ranging from minutes to hours (Blanchard and Blanchard, 1969). The freezing response can be elicited by acute threats such as exposure to a cat or footshock or by a CS presentation following aversive conditioning procedures involving footshock (Blanchard and Blanchard, 1969) (Fanselow and Helmstetter, 1988). Freezing can occur as a long bout of total immobility or as discrete episodes of freezing interupted by head swiveling or walking short distances. The fact that benzodiazepines, which are classic anti-anxiety drugs in humans, abolish or significantly reduce freezing to acute and conditioned stimuli serves as further evidence that freezing behavior is motivated by fear (Fanselow and Helmstetter, 1988) (Helmstetter, 1993).

It is worthwhile to note that freezing rats are neither flaccid nor asleep but can exhibit considerable muscle tone as well as reflex responses. A particularly striking example of this capacity comes from Griffith in 1920 [reported in (Fanselow, 1991)]. Griffith reported that he witnessed a rat, which was exploring the lid of its home cage, hang upside down from the food hopper for 22 minutes following a brief exposure to a cat. Despite the immobility, freezing rats are capable of autonomic responses (increases in arterial pressure) and acoustic startle responding (LeDoux et al., 1988) (Plappert et al., 
1993). These examples indicate that freezing rats are quite alert and responsive to their environment.

A number of brain regions are involved in generating the freezing response to a threat, either acute or conditioned (Figure 2). Lesion studies demonstrate that the ventral PAG is absolutely critical for the generation of the acute and conditioned freezing responses (Kim et al., 1993) (Fanselow, 1991). Not surprisingly, given its central role in fear related behaviors, the amygdala also plays a crucial role in behavioral inhibition. Lesions of the amygdala complex abolish or attenuate conditioned as well as unconditioned freezing (Kim et al, 1993) (Phillips and LeDoux, 1992). Lesions of the hippocampus disrupt the generation of conditioned freezing responses, but not freezing to acute footshock (Kim et al, 1993) (McNish et al., 1997). The hippocampus is involved in contextual conditioning, since lesions of it impair freezing to contextual cues and not explicit cues such as a sound (Phillips and LeDoux, 1992). Lastly, there is some evidence that the BNST has a role in generating the acute freezing response since lesions of that nucleus tend to reduce freezing as well (Schulz and Canbeyli, 1999).

The CRHergic system is involved in the freezing response. CRH microinjected into the ventricles of the brain increases freezing behavior to acute footshock and conditioned stimuli and is blocked by CRH antagonists, $\alpha$-helical CRH and antalarmin (Kalin et al, 1988) (Deak et al., 1999) (Kalin and Takahashi, 1990). Additionally, CRH antagonists injected into the amygdala attenuate the freezing response to footshock (Swiergiel et al., 1993). On the other hand, activation of the serotonergic system tends to reduce freezing. Systemic adminstration of citalopram and ipsapirone dose dependently attenuates the 


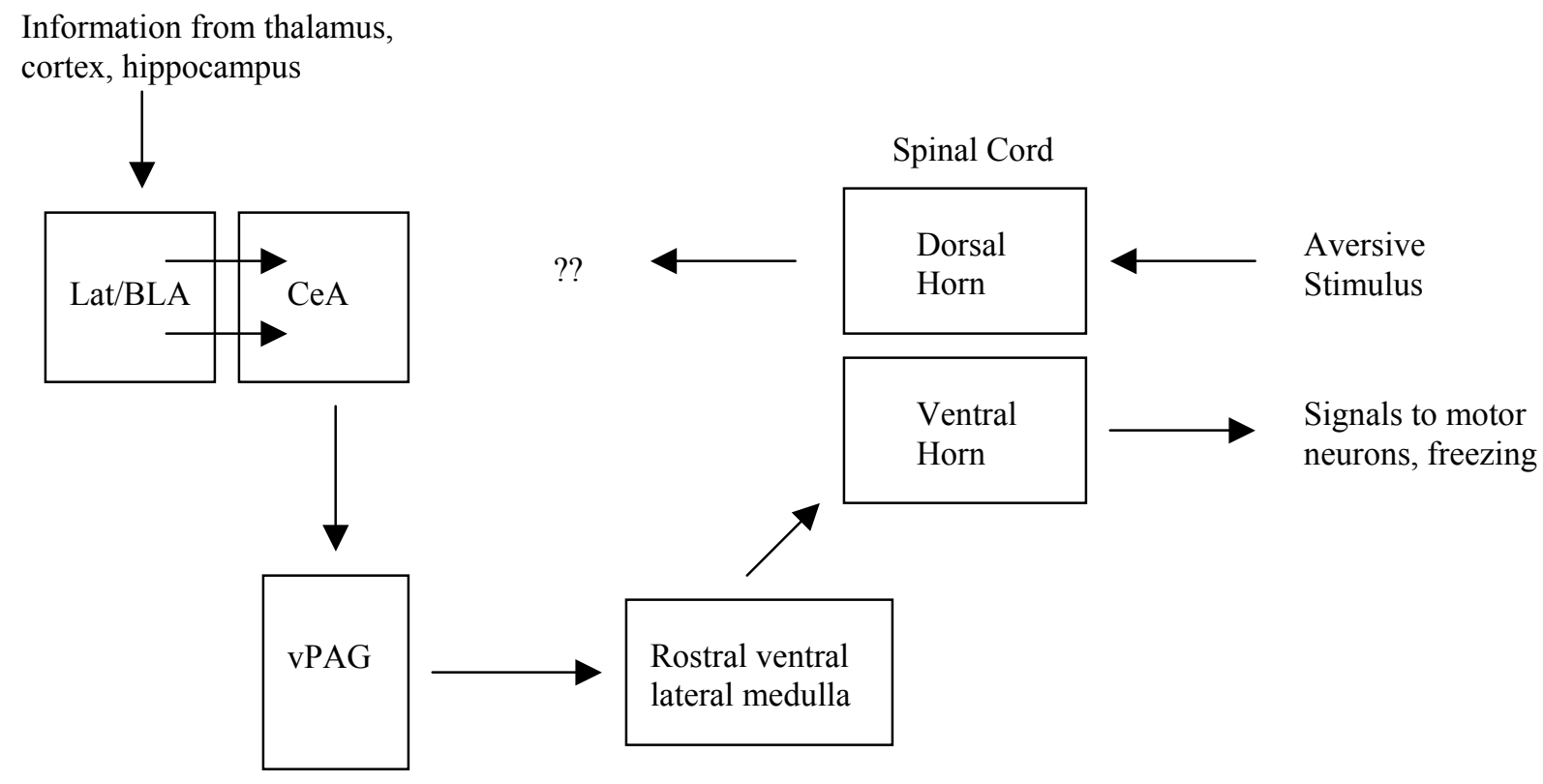

Figure 2. A simplified diagram describing the basic neuroanatomy of the freezing response. The figure is adapted from one by Fendt \& Fanselow, 1999. The diagram shows the key nuclei involved in generating the freezing response and shows where information from conditioning paradigms may enter the system. For example, aversive stimuli entering the pathway via the dorsal horn of the spinal cord might be tactile information from a footshock. Information converging on the Lat/BLA nucleus of the amygdala might ultimately come from sensory information such as light cues, auditory cues or contextual cues. Please note that this pathway shares some features with the ASR pathway. (Abbreviations: Lat/BLA, lateral and basolateral nuclei of the amygdala; CeA, central nucleus of the amygdala; vPAG, ventral periaqueductal gray) 
freezing response to conditioned stimuli (Inoue et al., 1996). And finally, chronic fluoxetine treatment reduces freezing behavior to conditioned stimuli but not the neuroendocrine responses such as increased plasma corticosterone (Zhang et al., 2000).

Corticosteriods play a pivotal role in freezing behavior. In fact, corticosterone is required for pre-weanling rats to even acquire the ability to freeze as indicated by the effects of adrenalectomy on young rats (Takahashi, 1994) (Takahashi and Kim, 1995). Pups adrenalectomized on post-natal day 10 , do not freeze when faced with a unfamiliar adult male rat (Takahashi and Kim, 1995). On the other hand, pups adrenalectomized on post-natal day 18 can freeze in that situation (Takahashi and Kim, 1995). The influence of corticosteriods on freezing behavior is also evident in adult animals. Kalin and colleagues reported that the time spent freezing following exposure to a human intruder by both infant Rhesus monkeys and their mothers positively correlated with plasma corticosterone levels (Kalin et al., 1998).

There are two published reports describing freezing behavior in PS rats. Takahashi and colleagues found that prenatal stress enhances freezing to a brief footshock (Takahashi et al., 1992a). These findings are not surprising since PS rats are known to have a hyperactive HPA axis, enhanced CRH release from the amygdala and alterations in the serotonergic system (Takahashi et al, 1990) (Cratty et al, 1995) (Peters, 1986a).

\section{Chronic Mild Stress}

Chronic stress in humans correlates well with the appearance of depressive symptoms (Kessler, 1997). One of the core symptoms of major affective disorder is anhedonia, or lack of finding pleasure in pleasurable things (American Psychological 
Association Diagnostic and Statistical Manual of Mental Disorders (DSM-IV)1995). An animal behavioral paradigm seeking to model this symptom of depression has been proposed by Paul Willner. Willner and colleagues have demonstrated that chronically stressed rats show decreased sucrose intake after several weeks of stress exposure compared to their pre-stress baseline values (Willner, 1997) (Willner et al., 1998). As Willner has shown, non-stressed rats prefer sucrose solutions over plain water and then, after repeated exposure to a series of mild stressors, sucrose intake declines. Furthermore, administration of a variety of drugs that have antidepressant activity in humans restores sucrose intake to pre-stress baseline values in chronically stressed rats (Papp et al., 1996) (Monleon et al., 1995). Two examples of drugs that increase sucrose intake under conditions of chronic mild stress are imipramine and buspirone. In contrast, drugs without anti-depressant activity do not have such an effect, for example haloperidol and chloprothixene (Papp et al, 1996).

Describing the neural circuitry contributing to depression or even decreased sucrose intake under conditions of chronic stress is considerably more difficult than with "simple" responses such as acoustic startle or freezing. This fact notwithstanding, several remarks can be made regarding this issue, particularly as they may apply to depression and the neurotransmitter systems discussed previously.

The CRHergic system certainly appears to be involved in depressive illness. CRH is increased in the cerebrospinal fluid of many depressed patients (De Bellis et al., 1993) (Smith and Nemeroff, 1988). Plasma cortisol is also found to be increased in many depressed patients but it can be decreased in specific forms of depression, compared to non-depressed controls (Jiang et al., 2000) (Bouwer et al., 2000). Finally, the number of 
neurons expressing $\mathrm{CRH}$ was found to be increased in the PVNH of depressed patients who died of causes other than suicide (Raadsheer et al., 1994). The serotonergic system is also changed in depression. Platelet binding of ${ }^{3} \mathrm{H}$-paroxetine and platelet concentration of 5HT are decreased (Alvarez et al., 1999) (Figueras et al., 1999). Serotonin transporter sites are also decreased in the prefrontal cortex of depressed patients who died of natural causes as well as the prefrontal cortex of suicide victims (Mann et al., 2000).

Finally, changes in elements of both the CRHergic and serotonergic systems can be affected by drug therapy, presumably for the benefit of the patient (De Bellis et al, 1993) (Alvarez et al, 1999). Sorting out the precise nature of the CRHergic and serotonergic involvement in depression is difficult. The question arises: are the changes that have been found the cause of the depression or an effect of the depression? Perhaps both? Regardless, the findings detailed above implicate these two systems in the pathology of depression.

Although no studies have been reported, several observations suggest that PS rats may be more susceptible to the debilitating effects of chronic mild stress than CON rats. Numerous studies have described increased plasma corticosterone in PS rats as compared to $\mathrm{CON}$ rats following stressful interventions, including repetitive exposure to the same open-field (Peters, 1982) (Fride et al, 1986). Increased release of CRH from the amygdala and increased content has been noted several brain regions as a result of prenatal stress (Cratty et al, 1995). As mentioned previously, pathology within the serotonergic system is also believed to be involved in depression and PS rats are known to have differences in the serotonergic system when compared to CON rats. Peters reported changes in 5-HT and 5HIAA content and changes in $\left[{ }^{3} \mathrm{H}\right]-5$-HT binding as a result of prenatal stress (Peters, 
1986a). Taken together, these findings imply that PS rats may be more susceptible to a chronic mild stress paradigm and display behavioral signs indicative of "depression" such as depressed sucrose intake.

\section{Introduction Summary}

Prenatal stress in several species of mammals results in offspring that react differently to novel and stressful situations than offspring whose mothers were not stressed during gestation. An explanation for these observations lies in alterations of the neurobiology of the stress responsive systems. As described above, these stress responsive systems include the CRHergic and serotonergic neurotransmitter systems.

There are at least two ramifications of studying the prenatal stress syndrome. First, we gain a better understanding of the etiology and neurobiology of affective and anxiety related disorders in humans, not only in children, but adults as well. It is interesting to note that the studies of prenatal stress in humans have focused exclusively on children (and the ages were unspecified). Yet, animal studies, including those of non-human primates, show that the effects of prenatal stress are long-lived since many of those studies were conducted on adolescent and adult-aged animals. Understanding the neurobiological and behavioral sequelae caused by prenatal stress will hopefully point the way towards refined therapeutic interventions for affective and anxiety disorders.

Secondly, there is the theoretical consideration of understanding the neural circuitry related to fear and anxiety and how it may function. The behaviors described above, acoustic startle responding and freezing, are "simple" behaviors in that they are well described anatomically, quantifiable and readily modified by experience. As such, they 
provide a framework for understanding information processing in the brain and the net behavioral output. Prenatal stress is unlikely to alter the basic neural circuitry of these behaviors. Rather, prenatal stress is likely to produce subtle changes at the level of the neurotransmitters and the synaptic connections that is reflected in the behavioral response. The prenatal stress syndrome may provide the basis of a neural circuitry model demonstrating the natural plasticity of fearful behaviors.

The principal aim of this project is to explore the interaction of prenatal stress and basic behaviors related to fear: acoustic startle, fear-potentiated startle and conditioned freezing. Other parts of the project will begin to survey elements of signal transduction pathways, particularly $5-\mathrm{HT}_{1 \mathrm{~A}}$ receptors, that are involved in fear-related responses and may be affected by prenatal stress. Additionally, developmental aspects of PS rats will be investigated, particularly with regard to the CRHergic system, since this system contributes to fearful behaviors. There will also be a brief look at the possibility that prenatal stress may confer susceptibility to chronic mild stress. Together, the data generated in this project will contribute to a better understanding of the prenatal stress syndrome and how prenatal stress changes behavior. 


\section{DISSERTATION OBJECTIVES}

Research demonstrates that prolonged maternal stress during pregnancy can adversely affect the fetus leading to developmental and emotional problems later in life. Given the timing of the maternal stress it is plausible to expect differences in basic parameters of growth and the neurochemistry of PS and CON rats to be detectable as early as day one of life. Logically, neurochemical differences, such as changes in CRH content, may contribute to behavioral differences later in life. Additionally, PS rats have a welldocumented HPA axis dysregulation which may predispose them to the debilitating effects of chronic mild stress. Our laboratory has demonstrated that adult PS rats demonstrate a smaller increase in acoustic startle responding following high doses of yohimbine (e.g. 5

$\mathrm{mg} / \mathrm{kg}$ ) than do CON rats. A number of reports have shown that adult PS rats demonstrate behavioral responses indicative of increased fearfulness to novelty or stressful stimuli; thus, they may also demonstrate increased responding in fear-conditioning paradigms. With these basic thoughts in mind, the following seven objectives are proposed for study.

\section{Part A, Developmental Analyses}

1. Perform an evaluation of gestational and growth parameters to determine if differences exist between PS and CON rats.

Method:

a) Retrospective study of length of gestation and litter size at P1

b) Measure body weights and ano-genital distance from P1 through adulthood. 
Hypothesis: Chronic maternal stress during pregnancy will decrease length of gestation, litter size, body weights and ano-genital distance.

2. Measure $\mathrm{CRH}$ content in the CeA/BLA, PVNH, BNST and DRN from early life through adulthood to determine when differences in CRH content occur. Method:

a) Micropunch these regions and perform CRH RIA in pre-weanling animals

b) Micropunch these regions and perform CRH RIA in post-weanling and young adult animals

Hypothesis: Differences in CRH content detected in older, adult animals originate as a result of the applied maternal stress and are detectable as early as the first day of life.

Part B, Susceptibility to Chronic Mild Stress

3. Determine if PS rats are more susceptible to the effects of Chronic Mild Stress.

a) Sucrose intake measurements in the chronically stressed rats

b) Sucrose intake measurements in naïve PS and CON rats

c) Adrenal weights in the same rats from part A and age-matched, naïve PS and CON rats

Hypothesis: Chronically stressed PS rats will have decreased sucrose intake, and larger adrenal glands than chronically stressed CON rats.

Part C, Acoustic Startle and Serotonergic Modulation of Acoustic Startle

4. Determine if differences between PS and CON rats exist in acoustic startle responding over a range of decibel levels. 
a) Peak startle responses over a range of decibel levels (80-125 dB SPL)

b) Latency to peak startle response over a range of decibel levels (80-125 dB SPL)

Hypothesis: PS rats will have greater peak startle responses and faster latencies than $\mathrm{CON}$ rats at higher decibel levels.

5. Determine if the $5-\mathrm{HT}_{1 \mathrm{~A}}$ receptor system is functionally affected by prenatal stress. Method:

a) 8-OH-DPAT modulated ASR

b) WAY-100635 modulated ASR

Hypothesis: $P S$ rats will be more sensitive to serotonergic modulation of acoustic startle responding than the CON rats.

Part D, Fear-Conditioned Responses

6. Determine if PS rats have greater fear-conditioned responses than CON rats. Method:

a) fear-potentiated startle

b) fear-condititioned freezing

Hypothesis: PS rats have more fearful reactions to novel and stressful stimuli so they will demonstrate a greater potentiated startle and conditioned freezing than CON rats.

\section{Part E, Signal transduction mechanisms}

7. Measure 5-HT $1 \mathrm{~A}$ receptor binding to determine if differences exist between PS and CON rats that may help explain behavioral differences.

Method: 
a) Receptor binding in block dissections of amygdala and hippocampus of PS and CON rats aged $\mathrm{P} 60$ to $\mathrm{P} 80$.

b) Basal and forskolin-induced cAMP production in block dissections of amygdala and hippocampus of PS and CON rats aged P60 to P80.

Hypothesis: Behavioral differences in PS rats are related to changes in signal transduction systems in one or more brain regions involved in those behaviors. 


\section{METHODS}

\section{Animals}

Male (225-250g) and female (225-250g) Sprague-Dawley rats to be used as mating pairs were purchased from Hilltop Labs, Inc. (Hilltop, PA). All animals were housed in the AALAC approved animal quarters at the Robert C. Byrd Health Sciences Center and all procedures performed had the approval of the Animal Care and Use Committee of WVU. The animals had food and water available ad libitum. Lights were turned on at 0800 hours and turned off at 2000 hours in the animal facility. All behavioral testing took place between the hours of 0900 and 1800, except for some of the sucrose intake assays as noted below. Tissue collection procedures took place between the hours of 0900 and 1230.

\section{Breeding and Induction of Prenatal Stress}

A male rat and a female rat were paired for six nights. The appearance of vaginal plugs in the bottom of the cage was counted as Gestational Day 0 (GD0). The pairs were separated and the females were housed singly for the duration of pregnancy and the nursing period. Half of the pregnant dams were designated to be in the prenatally stressed (PS) group; the other half of the group was designated to be left untouched except for routine animal care $(\mathrm{CON})$. Beginning on GD14, the dams in the PS group were removed from their home cage and placed in a new cage briefly to receive a subcutaneous injection of 0.1 $\mathrm{ml}$ of $0.9 \%$ saline at the nape of the neck and then returned to their home cage. This procedure was repeated once daily, at random times of the day by different lab personnel, until the pups were born, about GD22. Within 24 hours of birth, all litters with greater than 12 pups were randomly culled to 12 pups each without regard to sex. Pups were weaned 
from the dams on postnatal day 22 and housed as same-sex sibling pairs or triplets. Behavioral and neurochemical experiments were only performed on the male offspring from the PS and CON dams.

\section{Behavioral Methods}

\section{Acoustic Startle Apparatus}

Two sets of acoustic startle equipment were available. One set was manufactured by Med Associates (MA) (St Albans, VT) and the other set by San Diego Instruments (SDI) (San Diego, CA). Both sets of equipment utilize personal computers to program test sessions as well as retrieve and store data. Additionally, both sets of equipment rely on piezo-electric transducers that convert animal movement into voltage readings. Several PS and $\mathrm{CON}$ animals could be tested during the same session since multiple cabinets were available for each set of equipment. The type of equipment used in a given experiment is noted in the figure legend.

\section{Acoustic Startle Testing (baseline startle response and drug studies)}

Rats were placed in the testing apparatus and, following a 5 minute acclimation period, were exposed to either 60 acoustic startle stimuli (baseline) or 120 acoustic startle stimuli (drug studies) of 95 decibels SPL with an inter-stimulus interval of 30 seconds. The stimuli were 50 milliseconds in duration with the minimal programmable rise/decay time. Startle response data were recorded for a 250 millisecond period beginning at the onset of the noise burst. Data from across all of the trials of the session were averaged to create a mean response for the rat. 
The following parameters from each trial were evaluated from the acoustic startle test sessions as well as from the fear-potentiated startle test sessions: peak amplitude, latency to peak response and null period average responding. The peak amplitude is the maximum voltage change recorded during the 250 millisecond recording period. The latency to peak response is the time, in milliseconds, that the rats needed to reach the peak amplitude. Finally, the null period average response is the average voltage change recorded prior to any stimulus presentation to the animal, either a noise or a light stimulus. The null period was 50 milliseconds with the MA equipment and 1 millisecond with the SDI equipment. The null period average response serves as an index of motor activity of the rat not elicited by an overt stimulus.

\section{Fear-Potentiated Startle Training and Testing}

Rats were trained in the same acoustic startle apparati described above. The methods were modeled after those of Campeau, Liang and Davis (Campeau et al., 1990). Following a 5 minute acclimation period, the rats were exposed to ten $0.5 \mathrm{~mA}$ shocks paired with a light which served as the conditioned stimulus. The shocks were delivered by a removable metal grid in the floor of the animal holder. The light was illuminated for 3.7 seconds, the shock being presented during the last 500 milliseconds and then both stimuli co-terminated. The inter-stimulus interval was randomly timed, averaging 2.8 minutes. Ten such pairings constituted a daily training session and rats were trained for 2 or 4 consecutive days for the experiments presented here (Figure 3).

Testing occurred in the same acoustic startle apparatus as the training and was conducted 24 hours after the last training session. Following the 5 minute acclimation 
period, rats were exposed to 60 acoustic startle stimuli of 95 decibels with an inter-stimulus interval of 30 seconds. During the first 30 trials only noise was presented but during the last 30 trials, 15 randomly chosen noise trials were presented in conjunction with the same light used during training. During these 15 light plus noise trials, the 50 millisecond noise burst was presented at the same time as the shock would have been during the training sessions. Only noise was presented during the remaining 15 trials of the second half of the test. Please see Figure 3 for further illustration of these methods.

\section{Conditioned Freezing Apparatus}

An operant conditioning chamber, Model 80000, from Lafayette Instrument Company (Lafayette, IN) was used to conduct the conditioned freezing experiments. Shock was delivered to the chamber via Lafayette's Model 82400-SS Master Shocker and the model 58020 Neon grid scrambler. The repeated, timed delivery of shock was accomplished using their Dual Repeat Cycle Timer, model 51012.

\section{Conditioned Freezing to Context}

Naïve PS and CON rats were tested one at time in a counter-balanced fashion.

After 5 minutes of exposure to the chamber, the animal received 3 scrambled shocks of 0.5 $\mathrm{mA}$ intensity for 1 second duration with an interval of 10 seconds. The rat was observed for 5 more minutes and then returned to his home cage. The following day, the rat was placed into the same chamber for 10 minutes but no shocks were presented. Both sessions were videotaped for later analysis by a blinded observer. Comparisons were made between the groups with regard to freezing behavior and rearing behavior observed during the pre- 
shock and post-shock periods for session 1 and the entire session 2 period 24 hours later. Freezing behavior was defined as the absence of movement except that required for respiration, although slight head movements were permitted. Rears were defined as lifting both front paws off the grid floor in order to sniff at a higher level. Please see Figure 4 for further illustration of these methods.

\section{Conditioned Freezing to a Light Cue}

The same conditioning apparatus previously described was used with the addition of a 40 watt, clear light bulb suspended approximately $12 \mathrm{~cm}$ above the plexiglass ceiling. The methods were modeled after those published by Philips and LeDoux (Phillips and LeDoux, 1992). Naïve CON and PS rats tested one at time in a counterbalanced fashion as before. On day 1 , following a 1 minute acclimation, the light was illuminated for 20 seconds. A footshock of $0.5 \mathrm{~mA}$ intensity and 0.5 seconds in duration was delivered during the last second of illumination. Another 60 seconds elapsed and the procedure was repeated. Cleaning between sessions was accomplished using $1 \%$ acetic acid. The following day, the rats were placed back into the chamber which was modified from the previous day by covering the floor and two walls with plastic sheeting and the use of $70 \%$ ethanol to clean between sessions. There was a 1 minute acclimation and then the light was illuminated for 20 seconds. Both sessions were videotaped for analysis by a blinded observer. Freezing and rearing behaviors were assessed on both days during the 20 second light exposure as well as a 20 second period just before light exposure. Please see Figure 4 for further illustration of these methods. 
Sucrose Intake Assay

Testing lasted for one hour and began at 0830 or 2030 hours so that diurnal variations in feeding behavior could be taken into account in the series of assays. The rats were singly housed for this hour and exposed to 2 bottles, one with tap water and another with $1 \%$ sucrose. The bottles were weighed before and after the test session, the difference representing the amount consumed in grams. Lastly, the rats were weighed before each assay.

$\underline{\text { II. Drug Study Methods }}$

Drugs

8-OH-DPAT HBr and WAY-100635 Maleate were purchased from Sigma, Inc. CRF and the CRF antagonist, D-Phe-CRF, were purchased from RBI, Inc.

Systemic Dosing Studies (Acoustic Startle)

These studies were a randomized cross-over design such that each rat received vehicle and several doses in the study. Drug washout periods were 24 or 48 hours and are indicated in the figure legends. Saline $(0.9 \%)$ was the vehicle used in all studies. All injections were given intraperitoneally (i.p.). 


\section{Fear-Potentiated Startle Methods}

A. Training Trial

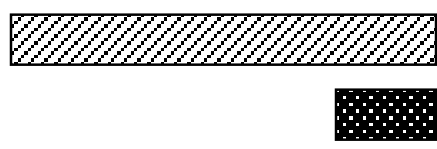

Light + Shock Trial

B. Testing Trials

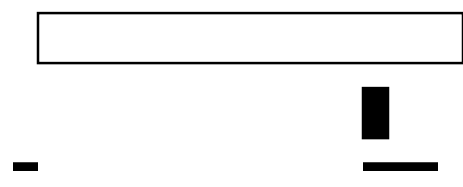

Noise Alone Trial

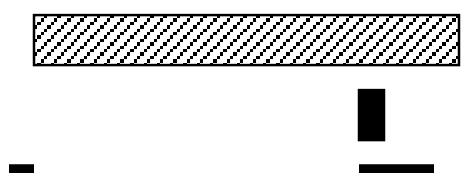

Light + Noise Trial

C. Test Session Map

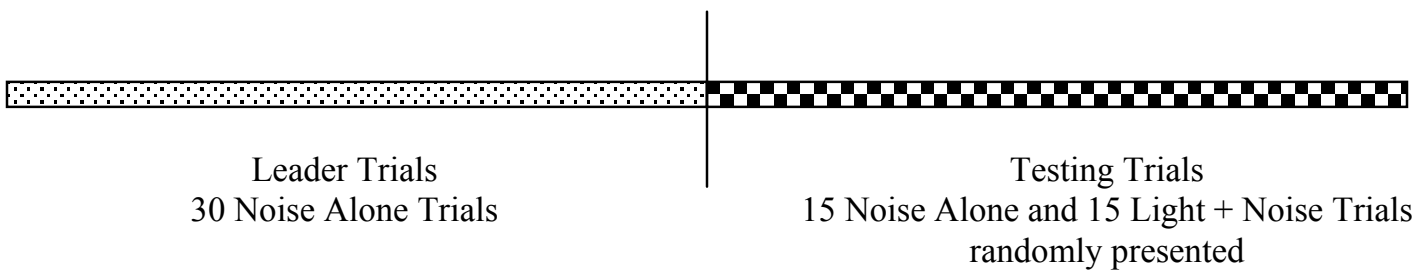

Figure 3. Schematic representation of the Fear-Potentiated Startle methods showing the temporal arrangement of the conditioned stimuli and unconditioned stimuli during the training and testing sessions. A) The ten training trials per session were identical and occurred on a random interval averaging 2.8 minutes. B) There were two types of trials, noise alone and light + noise, and the presentations occurred on a 30 second interval. The noise burst was presented at the same time as the shock would have been during training. C) A diagram showing the composition of a single test session. See Methods Section for more details. Please note that the diagrams in parts $\mathrm{A}, \mathrm{B}$ and $\mathrm{C}$ are not to scale.

Key For Parts A \& B

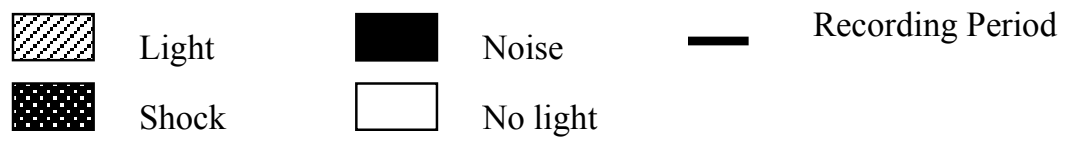




\section{Fear-Conditioned Freezing Methods}

A. Fear-Conditioned Freezing to Context

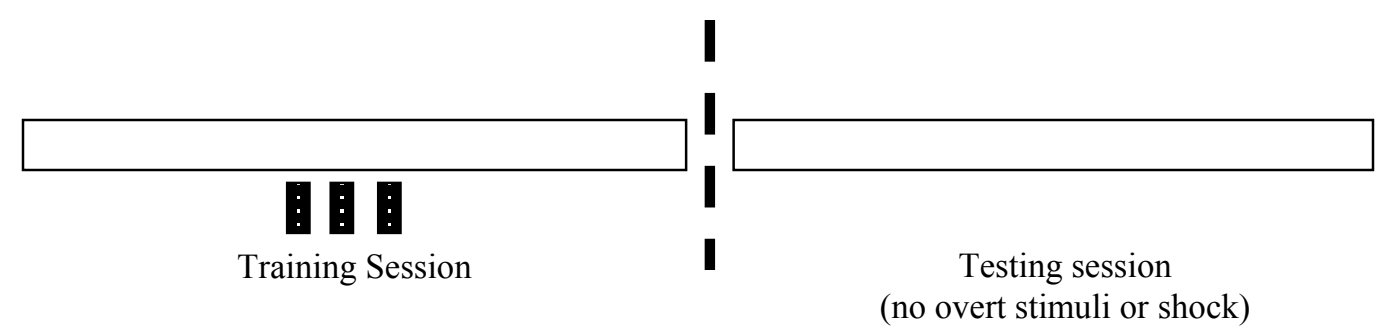

B. Fear-Conditioned Freezing to a Light Cue

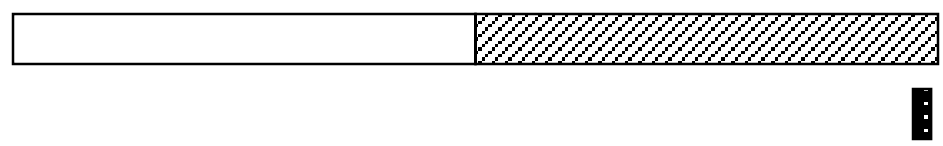

Training Trial

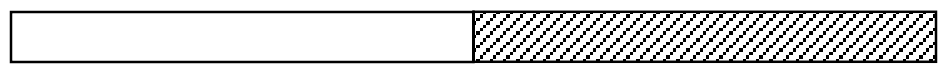

Testing Trial

Figure 4. Schematic representation of the fear-conditioned freezing methods showing the temporal arrangement of the conditioned stimuli and unconditioned stimuli in the training and testing sessions. A) There was one exposure to the testing chamber, with the three shocks presented in the middle of the time period. Testing was conducted 24 hours later in the same chamber, but no overt stimuli or shocks were presented. B) There were two training trials separated by a one minute period. Testing occurred 24 hours later in a different context, but used the same light as during the training. The "no light" period served to measure freezing to the context. See Methods section for more details. Please note that the diagrams in parts A and B are not to scale.

Key For Parts A \& B

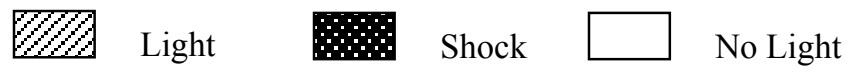




\section{Biochemical Methods}

\section{Tissue Collection and Dissection}

All tissue was collected between the hours of 0900 and 1230. Rats were sacrificed by decapitation. The brain was quickly removed, placed on its dorsal surface inside a plastic vial and frozen in liquid nitrogen. The brains were stored at $-80^{\circ} \mathrm{C}$ until use. For experiments using block dissected tissue, the brains were thawed and cut using a razor blade according to the method of Glowinski and Iversen (Glowinski and Iversen, 1966). The block of tissue was weighed and quickly placed into the appropriate ice-cold buffer. Other experiments utilized a micro-dissection technique. The frozen brains were mounted on a tissue stage and sectioned in the coronal plane at 200 microns using a Model HM505E Cryostat (Microm, Inc.) The sectioned tissue was thaw mounted onto a glass slide and then refrozen. Disks of frozen tissue were removed using 1 or $2 \mathrm{~mm}$ dissecting curettes. For tissue from P25 and older brains landmarks for micropunching specific nuclei were judged using the $2^{\text {nd }}$ edition atlas of Paxinos and Watson(Paxinos and Watson, 1982). Landmarks for micropunching nuclei in younger tissue were determined using the altlas of Sherwood and Timiras (Nancy M.Sherwood and Paola S.Timiras, 1970) as well as the atlas of Altman and Bayer for P1 tissue (Altman and Bayer, 1995).

\section{CRH Extraction Procedure}

CRH was extracted by homogenizing the micro-dissected tissue with a Model 60 Sonic Dismembrator (Fisher Scientific, Inc) at setting 3 for 10-15 seconds in a buffer of $0.05 \mathrm{~N} \mathrm{HCl}, 1 \mathrm{mg} / \mathrm{ml}$ Ascorbate and $0.1 \%$ Triton X-100. The samples were heated in a $70^{\circ} \mathrm{C}$ water bath for 10 minutes and then frozen overnight. The next day, the samples were 
thawed and $100 \mu \mathrm{L}$ aliquot was removed for protein concentration measurement. The remainder of the sample was centrifuged at $3000 \mathrm{~g}$ for 25 minutes. Finally, $300 \mu \mathrm{L}$ of the supernatant was dried down in a Speed Vac Concentrator (Savant, Inc) and then stored at $80^{\circ} \mathrm{C}$ until use. Protein concentrations were determined by the method of Lowry et. al.(Lowry et al., 1951).

CRH RIA

Lyophilized tissue samples were reconstituted in $1 \mathrm{ml}$ of RIA buffer $(0.03 \mathrm{M}$ Sodium Phosphate, 0.01M Sodium EDTA, 0.1\% Triton X-100, 0.2 Units/ml Aprotinin). Samples were incubated at $4^{\circ} \mathrm{C}$ for 24 hours with rabbit derived anti-CRH antibody (RC70, compliments of Wiley Vale, Salk Institute, CA). Excess ${ }^{125}$ I-labeled CRH was then added and the samples were incubated for another 24 hours at $4^{\circ} \mathrm{C}$. Next, the samples were incubated for 20 minutes in the presence of sheep anti-rabbit $\mathrm{IgG}$ antibody and $0.14 \mathrm{~g} / \mathrm{ml}$ of Polyethylene Glycol (6,000-8,000 MW). Finally, the samples were centrifuged for 20 minutes at $5000 \mathrm{rpm}$ in a Sorvall centrifuge and the supernatant discarded. Pellets were counted in a 1470 Wizard Gamma Counter (Wallac, Inc) and were compared against known concentrations of $\mathrm{CRH}$.

\section{Membrane Preparation Procedure for Radioligand Binding}

The block dissected tissue samples were homogenized using a Model 60 Sonic Dismembrator (Fisher Scientific, Inc) at setting 4 for $20-30$ seconds in $3.5 \mathrm{~mL}$ of $320 \mathrm{mM}$ sucrose and $5 \mathrm{mM}$ Tris- $\mathrm{HCl}$ at $\mathrm{pH}$ 7.4. The homogenate was centrifuged at $100 \mathrm{~g}$ for 10 minutes at $4^{\circ} \mathrm{C}$. The supernatant was saved and the pellet washed in the same buffer by 
centrifugation as before. This second supernatant was pooled with the first supernatant and the entire volume was centrifuged at $70,000 \mathrm{~g}$ for 25 minutes at $4^{\circ} \mathrm{C}$. The pellet was resuspended in $\sim 10$ volumes of $50 \mathrm{mM}$ Tris- $\mathrm{HCl}$ at $\mathrm{pH} 7.4$. Aliquots were frozen at $-80^{\circ} \mathrm{C}$ for later use.

\section{$\left[{ }^{3} H\right]$ 8-OH-DPAT Binding Studies}

Aliquots of membranes $(50-80 \mu \mathrm{g})$ were incubated with $50 \mathrm{mM}$ Tris- $\mathrm{HCl}(\mathrm{pH} 7.4)$ containing various concentrations of ${ }^{3} \mathrm{H}-8-\mathrm{OH}-\mathrm{DPAT}(0.10-50 \mathrm{nM})(\mathrm{NEN}$ Dupont, Inc). The incubation was 30 minutes at room temperature. The reaction was terminated by rapid filtration over Whatman GF/B filters followed by 3 washes with $3.5 \mathrm{ml}$ of the same icecold buffer. Non-specific binding was determined in the presence of $10 \mu \mathrm{M} 5$-HT HCl. Protein concentrations of the final dilution used in the assay were determined by the method of Lowry et. al.(Lowry et al, 1951).

\section{Tissue Preparation for cAMP Studies}

Blocks of tissue were collected as described above for the block dissections used in the receptor binding experiments. The dissected tissue was placed into $2 \mathrm{~mL}$ of buffer containing: $80 \mathrm{mM}$ Tris $\mathrm{HCl}, 300 \mathrm{mM}$ sucrose, EGTA 1mM, $\mathrm{Na}_{2}$ EDTA 5mM, dithiothreitol $5 \mathrm{mM}$ and the $\mathrm{pH}$ was adjusted to 7.4. The tissue was homogenized by hand in glass-on-glass homogenizers (Pyrex) and then centrifuged at 50g for 5 minutes. The supernatant was collected and centrifuged for 10 minutes at $39,000 \mathrm{~g}$. The final pellet was resuspended in 500 microliters of homogenization buffer and stored in 100 microliter 
aliquots at $-80^{\circ} \mathrm{C}$ until use. A sample of the resuspended pellet was taken for protein determination by the method of Lowry et. al. (Lowry et al, 1951).

\section{cAMP Studies}

Thawed tissue samples containing 100 to $120 \mu \mathrm{G}$ of protein were incubated at $37^{\circ} \mathrm{C}$ for 10 minutes in the following buffer: Tris $\mathrm{HCl} 200 \mathrm{mM}$, EGTA $1 \mathrm{mM}$, MgAcetate $5 \mathrm{mM}$, 3-isobutyl-1-methylxanthine $0.5 \mathrm{mM}$, ATP $\left(2 \mathrm{Na}^{+}\right) 1 \mathrm{mM}$, GTP $\left(\mathrm{Na}^{+}\right) 10 \mu \mathrm{M}$ and the appropriate cocentration of drugs. Stock forskolin was obtained from Sigma-Aldrich, Inc and initially dissolved in dimethylsulfoxide before a 1:1000 final dilution in the buffer described above. The reaction (500 $\mu \mathrm{L}$ final volume) was started with the addition of the tissue and terminated by exposure to a $95^{\circ} \mathrm{C}$ water bath for 3 minutes. Following inactivation, the sample was diluted $1: 20$ and stored at $-20^{\circ} \mathrm{C}$ until the cAMP assay. A 50 $\mu \mathrm{L}$ aliquot of each reaction was assayed in duplicate. The amount of cAMP was determined using an enzyme-immunoassay (EIA) kit manufactured by IBL-Hamburg (Cayman Chemical, Inc.). The manufacturer's instructions were followed for nonacetylated standards and samples. Additionally, the manufacturer's test results state that the kit was specific for the detection of cAMP and would not cross react with other cyclic nucleotides.

\section{Statistical Analysis}

Statistical analysis was performed as indicated under the Results section using GBStat ${ }^{\circledR}$ Version 7.0 (Dynamic Microsystems, Inc). For experiments with 2 groups, Student’s t-tests were performed. For experiments with multiple groups, ANOVA or repeated 
measures ANOVA was performed with post-hoc analysis using Fisher's Least Significant Difference (LSD) protected t-test, unless otherwise stated. GraphPad Prism ${ }^{\circledR}$ Version 3.0 (GraphPad Software, Inc) was used for non-linear regression fitting and comparisons of those regressions where necessary. A p value of 0.05 or less was considered significant for statistical comparisons. 


\section{RESULTS}

\section{Part A, Parameters of Development}

A retrospective study of our records of 72 CON litters and 71 PS litters was conducted (Table 1). These litters were conceived and born over a two year period from 1998 to 2000 . The study showed that length of gestation was not different between the groups (Student's t-test; $\mathrm{t}=0.10, \mathrm{p}<0.55$ ) and the total litter size was not different between the groups on postnatal day 1 (Student's t-test; $\mathrm{t}=0.09, \mathrm{p}<0.99$ ). Additionally, the gender ratio of these same litters was not affected by our random culling procedure, as determined at weaning (Student's t-test, $\mathrm{t}=0.20, \mathrm{p}<0.39$ and $\mathrm{p}<0.62$ for males and females respectively). The distribution of litter size at birth was also reviewed in the same study (Table 2a). Litters sizes determined at the P1 culling event were sorted into 3 groups: $<9$ pups, 10 to 19 pups and $>19$ pups. The CON group had 5 more litters with $>19$ pups and, consequently, had a greater number of dead pups discovered at the P1 culling event on a per litter basis. Chi-square analysis of the data in Table 2 a did not reveal a difference between the groups when litter sizes were distributed this way $\left(\chi^{2}=0.01, \mathrm{p}<0.99\right)$.

Body weight varied as a function of litter size at the P1 age point (Table $2 \mathrm{~b}$ ). Since no differences were detected between the groups in these parameters, group data were averaged together. Additionally, data were not available for litter sizes less than 12 pups at the P1 age. Body weights were smaller with larger litter sizes ( 1 factor $\operatorname{ANOVA}_{(2,23)}=$ 14.82, $\mathrm{p}<0.0001)$. AG distance was not evaluated in this respect.

The weight and ano-genital distance of PS and CON rats were compared as a function of age in experimentally naïve rats (Figures 5 and 6). Figure 5 shows body weight and ano-genital distances taken at four pre-weanling ages. No statistical differences could 
be found at these ages between the two groups ( 2 factor ANOVA; $\mathrm{F}_{(1,292)}=0.90$ for weights and $\mathrm{F}_{(1,292)}=0.312$ for AG Distance). There is the expected age-related increase in these parameters $\left(2\right.$ factor ANOVA; $\mathrm{F}_{(3,292)}=2158, \mathrm{p}<0.0001$ for weights and $\mathrm{F}_{(3,292)}=2412, \mathrm{p}$ $<0.0001$ for AG Distance). Figure 6 shows body weight and AG distance measurements taken at three post-weaning ages. As with the pre-weanling pups, no statistical differences were detected between the groups ( 2 factor ANOVA; $F_{(1,62)}=1.40$ for weights and $F_{(1,62)}=$ 0.32 for AG Distance). However, there was an age-related increase in both parameters (2 factor ANOVA; $\mathrm{F}_{(2,62)}=917, \mathrm{p}<0.0001$ for weights and $\mathrm{F}_{(2,62)}=590, \mathrm{p}<0.0001$ for AG Distance).

Previous studies demonstrated that $\mathrm{CRH}$ content in the CeA/BLA and PVNH was increased in the PS group when the rats were $>90$ days of age. CRH content was measured in micro-punched samples containing these brain regions as well as the BNST from ages as early as P1 up to P21 in experimentally naïve rats. Figure 7 shows CRH content measurements in pre-weanling rats of both groups. No differences were detected in the BNST, PVNH or CeA/BLA at any age between the groups ( 2 factor ANOVA; $\mathrm{F}_{(1,46)}=1.95$ for BNST, $F_{(1,46)}=0.09$ for CeA/BLA, $F_{(1,45)}=0.00007$ for PVNH). However, there was an age related increase in $\mathrm{CRH}$ content in both groups ( 2 factor ANOVA; $\mathrm{F}_{(1,46)}=31.27$, $\mathrm{p}<0.0001$ for BNST, $\mathrm{F}_{(1,46)}=22.59, \mathrm{p}<0.0001$ for $\mathrm{CeA} / \mathrm{BLA}, \mathrm{F}_{(1,45)}=30.66, \mathrm{p}<0.0001$ for PVNH).

Figure 8 shows CRH content measurements in micro-punched samples of BNST, BLA/Lat and PVNH from experimentally naïve post-weanling and young adult-aged rats. Again, no differences were detected between the groups within any brain regions ( 2 factor ANOVA; $\mathrm{F}_{(1,43)}=1.04$ for BNST, $\mathrm{F}_{(1,45)}=0.27$ for CeA/BLA, $\mathrm{F}_{(1,45)}=0.07$ for PVNH and 
$F_{(1,45)}=0.32$ for DRN). The age related increase seen in the pre-weanling rats was not detected in this data set ( 2 factor ANOVA; $\mathrm{F}_{(1,43)}=0.87$ for BNST, $\mathrm{F}_{(1,45)}=2.25$ for $\mathrm{CeA} / \mathrm{BLA}$ and $\mathrm{F}_{(1,45)}=1.57$ for PVNH and $\mathrm{F}_{(1,45)}=1.66$ for $\left.\mathrm{DRN}\right)$. 
Table 1.

\begin{tabular}{|c|c|c|}
\hline Parameter & CON & PS \\
\hline Length of Gestation (days) & $21.96 \pm 1.02$ & $21.85 \pm 0.99$ \\
\hline No. Pups at P1 & $17.07 \pm 4.57$ & $17.08 \pm 4.73$ \\
\hline No. Females at Weaning & $6.19 \pm 1.57$ & $5.96 \pm 1.84$ \\
\hline No. Males at Weaning & $5.16 \pm 1.57$ & $5.54 \pm 1.77$ \\
\hline
\end{tabular}

Table 1 shows several parameters of litter development. 72 litters and 71 litters were surveyed for the CON and PS groups, respectively. No statistical differences were detected in any of the parameters. Values are means \pm S.D. (see results for details). 
Table 2a.

\begin{tabular}{|c|c|c|}
\hline Parameter & CON & $P S$ \\
\hline No. Litters with $>19$ pups & 21 & 16 \\
\hline No. Litters with $10-19$ pups & 50 & 52 \\
\hline No. Litters with $<9$ pups & 1 & 3 \\
\hline Death rate/litter $>19$ pups & 0.00987 & 0.00261 \\
\hline
\end{tabular}

Table 2a shows the distribution of litter size between the two groups. 72 litters and 71 litters were surveyed for the CON and PS groups, respectively. Differences were not detected in the distribution of litter size (see results).

Table $2 b$.

\begin{tabular}{|c|c|c|c|}
\hline Parameter & $14-16$ pups & $17-19$ pups & $22-27$ pups \\
\hline Body weight (g) & $6.53 \pm 0.05$ & $6.32 \pm 0.12$ & $5.36 \pm 0.09^{*}$ \\
\hline
\end{tabular}

Table $2 \mathrm{~b}$ shows the effect of litter size on body weight at P1. Litters with large numbers of pups at P1 are significantly smaller than litters with fewer pups at P1. CON and PS weights were averaged for inclusion in the table. There are 6 to 11 independent litters represented at each litter size. Values are weights \pm S.E.M. (See results for details; ${ }^{*} \mathrm{p}<0.01$ compared to $14-16$ pups) 


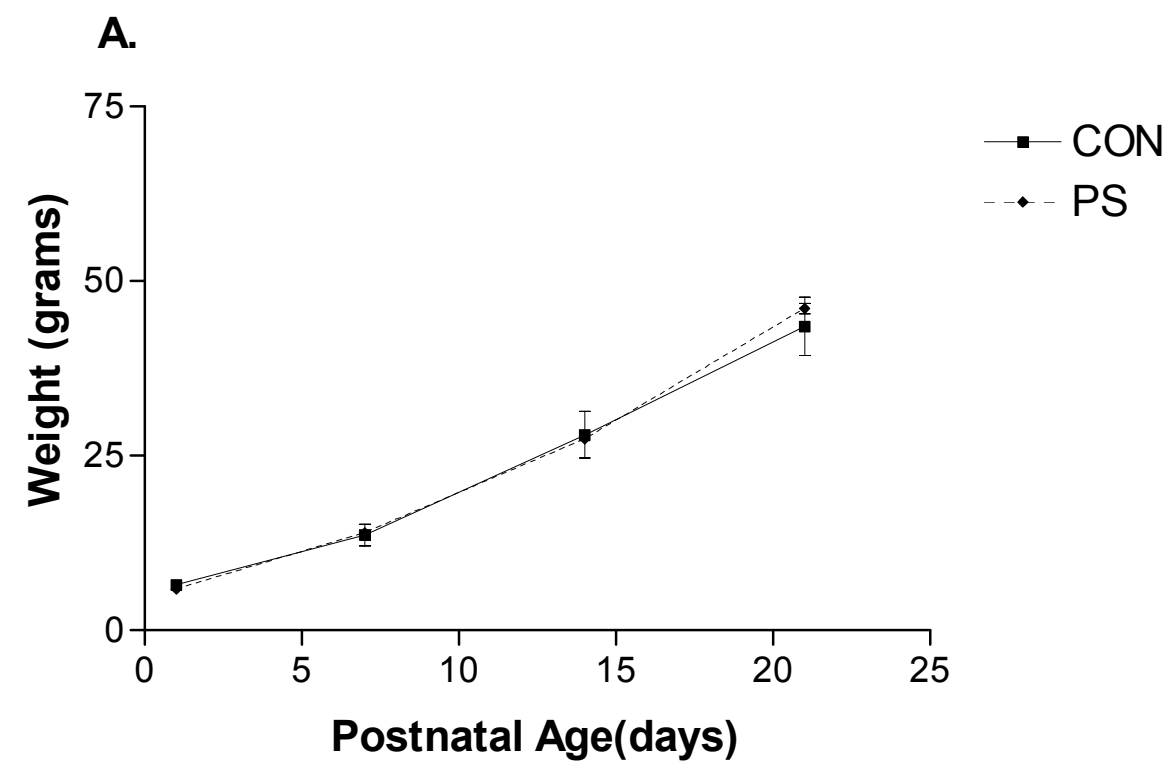

B.

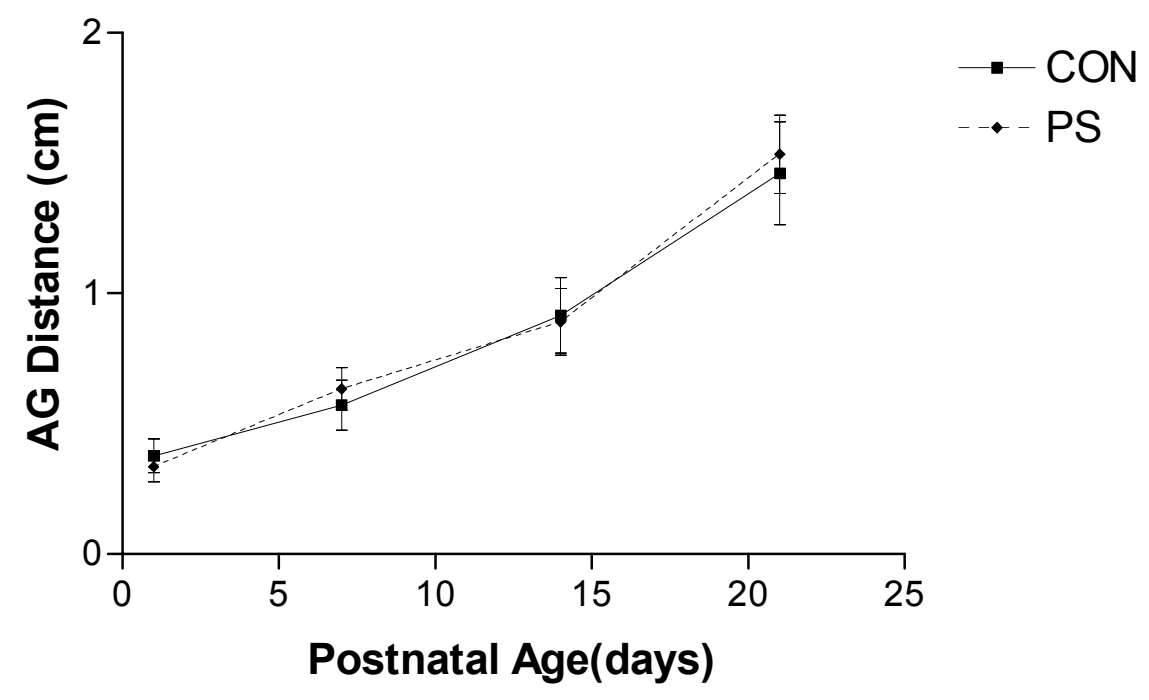

Figure 5. Changes in body weight and ano-genital distance with age in male pre-weanling PS and CON rats. Both parameters show an age related increase in these parameters but no between group differences were detected. Each data point represents the average of mean values from 18-68 individual rats taken from 8-10 independent litters. Values are means \pm S.D. (See results for statistical details). 


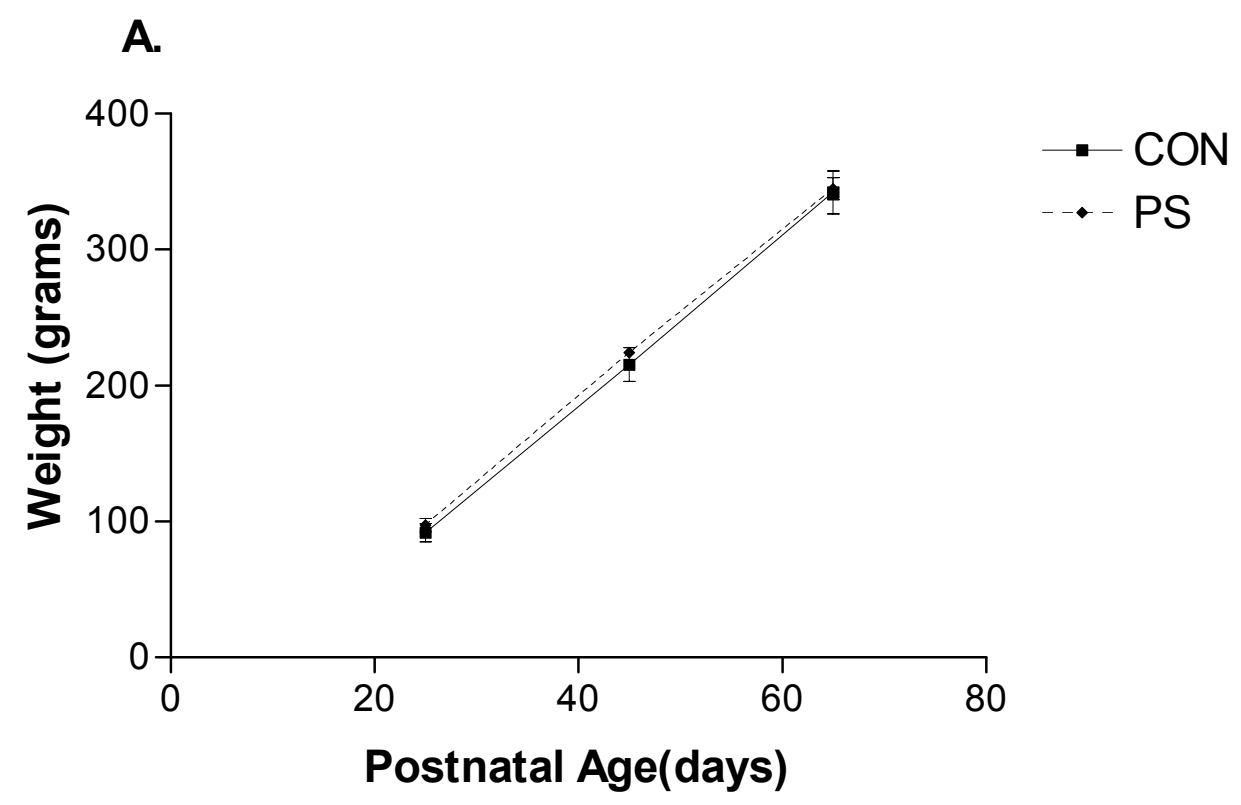

B.

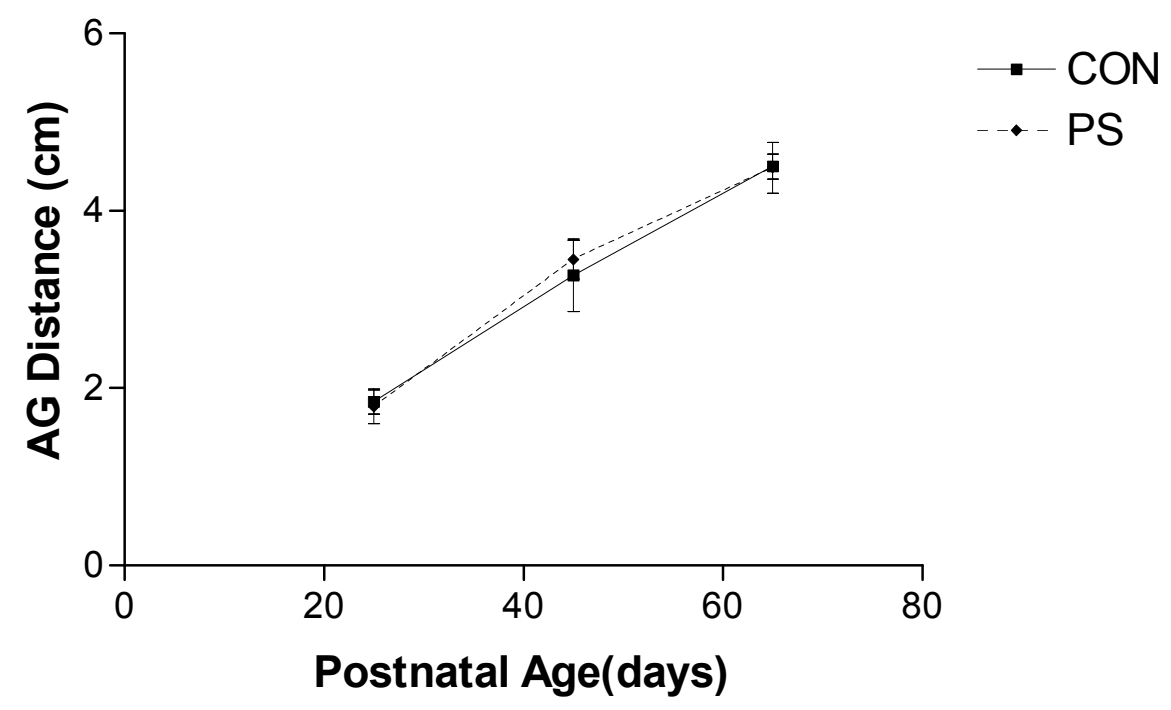

Figure 6. Changes in body weight and ano-genital distance with age in male PS and CON rats. Both parameters show an age related increase in these parameters but no differences were detected between the groups. Each data point represents 6-14 rats from 4-7 independent litters. Values are means \pm S.D. (See results for statistical details). 
A. BNST

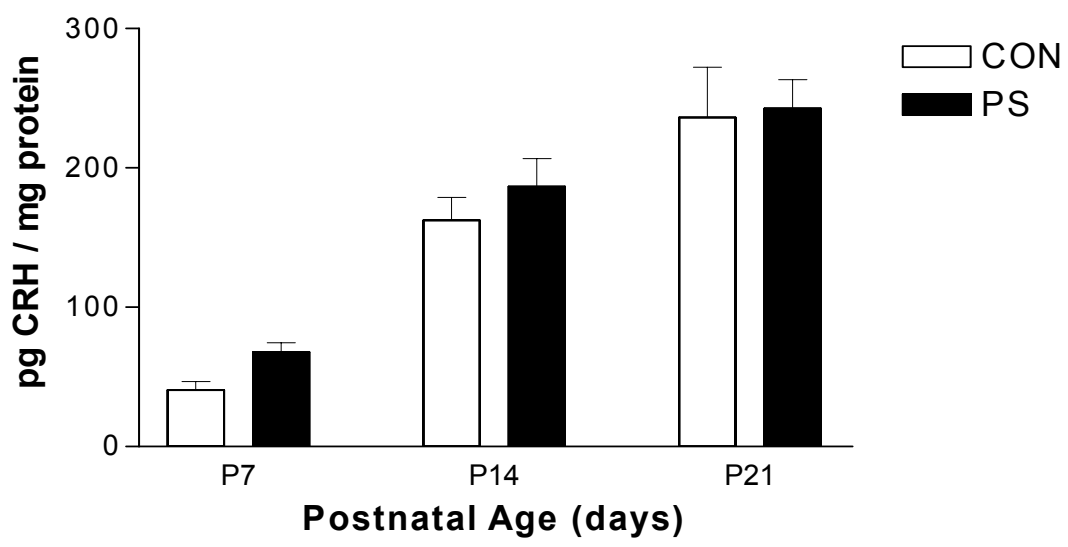

B. CeA/BLA

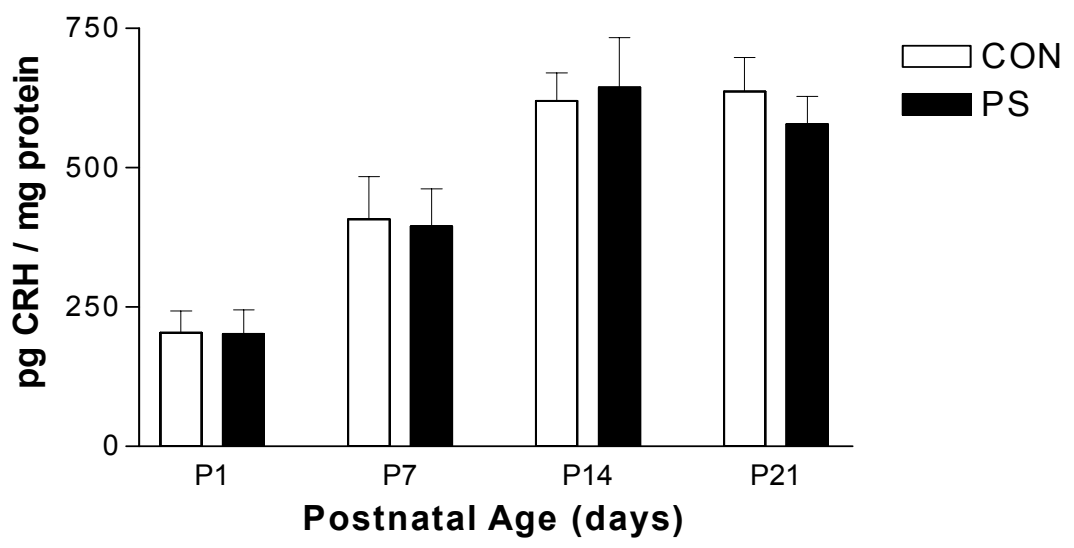

C. PVNH

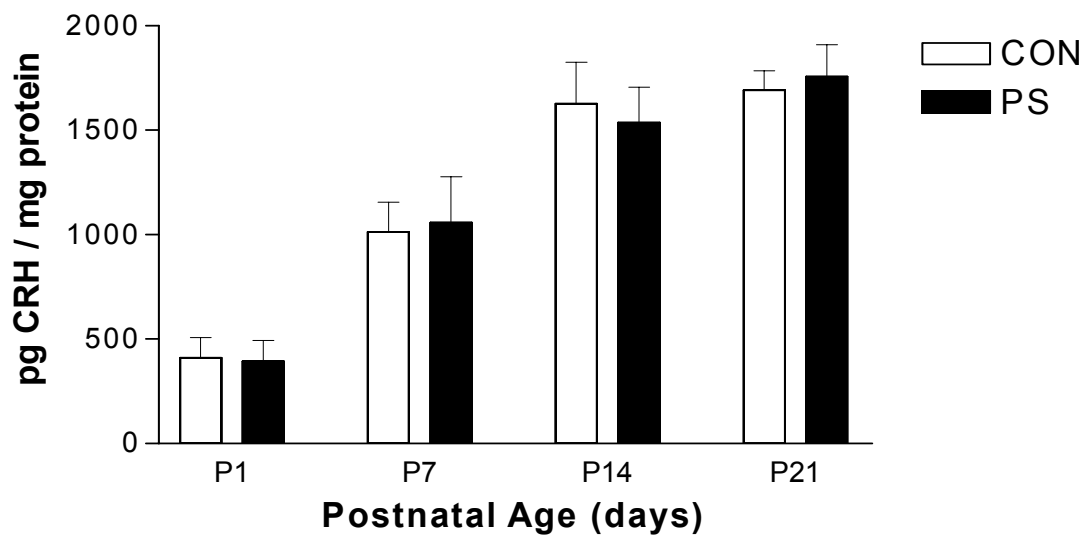

Figure 7. CRH content in pre-weanling $\mathrm{PS}$ and $\mathrm{CON}$ rats. A) CRF content in the BNST. Each data point cons is ts of measurements from 7-8 individual rats from independent litters. B) $\mathrm{CRH}$ content in the $\mathrm{CeA} / \mathrm{BLA}$. Each data point consists of 5-6 individual rats from independent litters. C) $\mathrm{CRH}$ content in the PVNH. Each data point consists of 5-6 individual rats from independent litters. No differences were detected between PS and CON rats at any age or in any brain region. An age related increase was detected in all three brain regions. Values in each graph are means \pm S.E.M. (See results for statistical details) 
A. BNST

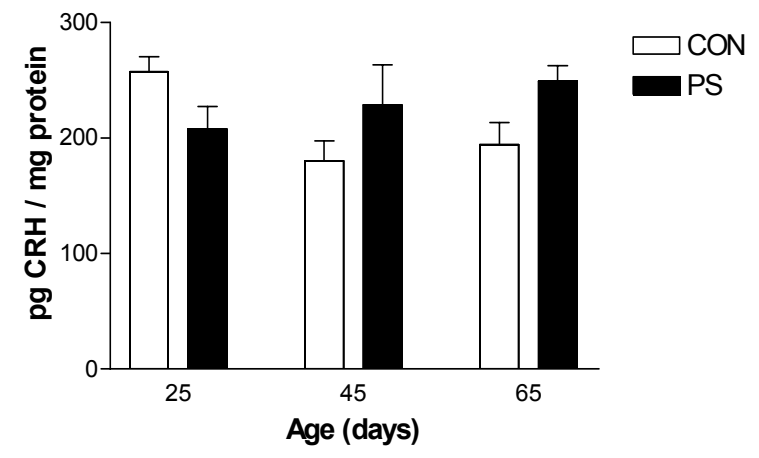

C. PVNH

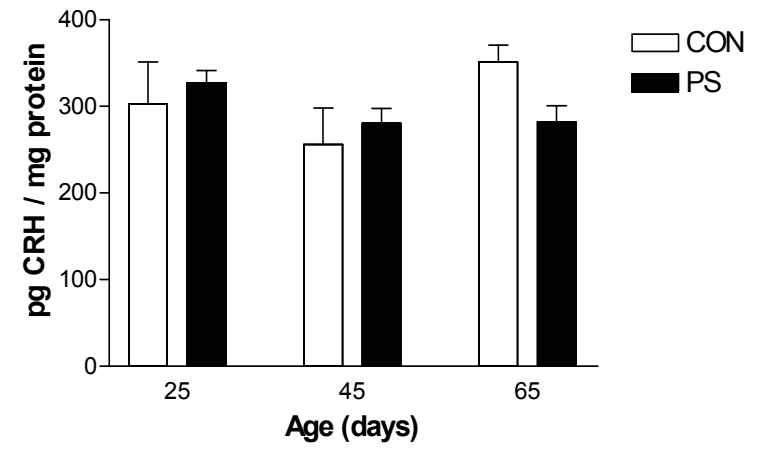

B. CeABLA

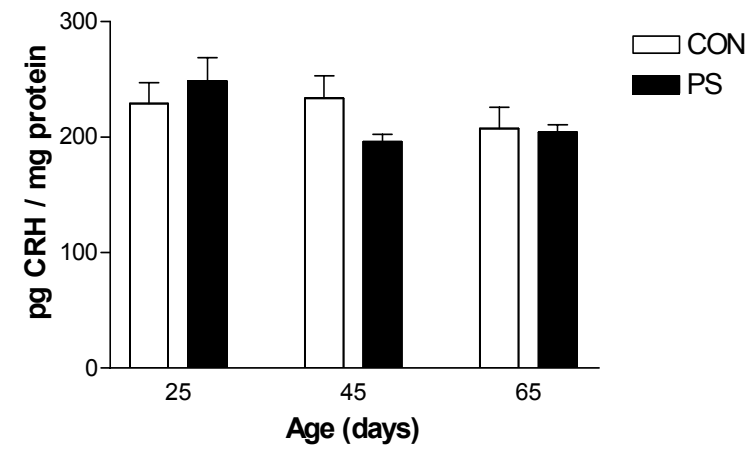

D. DRN

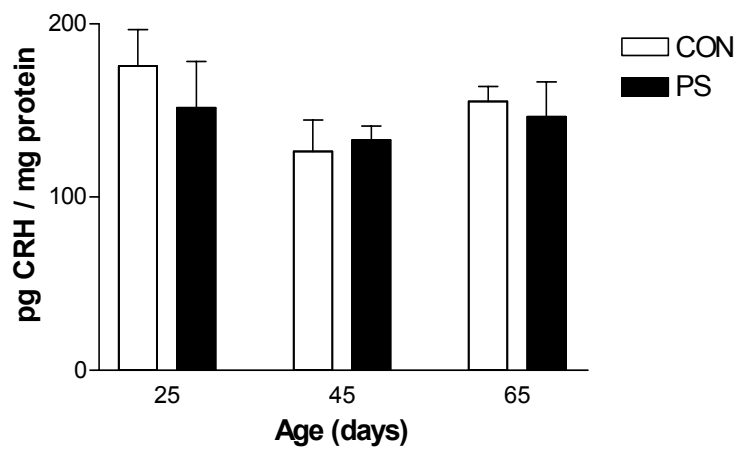

Figure 8. The graphs show pg $\mathrm{CRH} / \mathrm{mg}$ protein in 4 brain regions from naive post-weanling and young adult rats. No differences were detected in any brain region between the groups. Additionally there were no age related increases detected. The $\mathrm{n}$ value was 7-8 per region per group, representing 7-8 independent litters per group. Values are means \pm S.E.M. (See results for statistical details). 


\section{Part B, Preliminary Chronic Mild Stress Experiments}

PS and CON rats that were subjected to various experimental procedures from $\sim 25$ days of age to 120 days of age were assessed for the possibility that PS rats are more susceptible to the effects of Chronic Mild Stress. The nature of the experimental procedures is outlined in Table 3 . The behavioral index of stress was sucrose intake assayed once weekly for four weeks (Figure 9). The PS rats had significantly less sucrose intake than the CON rats during nighttime and daytime assays (RM 2 factor ANOVA; $\mathrm{F}_{(1,39)}=17.18, \mathrm{p}=0.0006$ for nighttime, $\mathrm{F}_{(1,39)}=4.69, \mathrm{p}=0.044$ for daytime). Post hoc analysis using of the nighttime intake data using Fisher's test showed that PS rats had less sucrose intake than the CON rats during week three. Additionally, a Student's t-test found that PS rats had less intake during week two $(\mathrm{t}=2.3, \mathrm{p}<0.02)$. Water intake was comparable between the groups during the nighttime and daytime assays (RM 2 factor ANOVA; $F_{(1,70)}$ $=1.05)$. Body weight was generally lower in the PS group over the course of conducting the assays, although both groups demonstrated an age related increase in body weight (RM 2 factor ANOVA; $F_{(1,99)}=7.09, p=0.0159$ for group, $F_{(1,99)}=20.46, p<0.0001$ for age). There was not a significant interaction between the group and age parameters suggesting that the two groups were gaining weight at the same rate $\left(\mathrm{RM} 2\right.$ factor ANOVA; $\mathrm{F}_{(1,99)}=$ 0.31). Post-hoc analysis using Fisher's LSD protected t-test did not detect specific differences between the groups at any time point with regard to body weight.

Another group of 8 naïve PS and CON rats was assessed for sucrose intake over four weeks as before to see if PS rats naturally had less sucrose intake. The average sucrose intake during both weeks during the daytime for the CON and PS rats was $0.90 \pm$ 0.29 and $1.37 \pm 0.48$ milligrams, respectively. During the nighttime assays, the average 
sucrose intake for the CON and PS rats was 3.76 \pm 1.07 and $2.30 \pm 0.61$ milligrams, respectively. Statistical analysis did not reveal an effect of group but did reveal an effect of time of day $(\mathrm{RM} 2$ Factor ANOVA; $\mathrm{F}(1,31)=0.96$ for group, $\mathrm{F}(1,31)=17.34, \mathrm{p}<0.0002$ for time of day). Water intake was comparable across both assay times and groups (data not shown).

At sacrifice, the adrenal glands were collected from these chronically stressed PS and $\mathrm{CON}$ rats as well as in a group of age-matched, experimentally naïve PS and CON rats (Table 4). Adrenal weights were not different across the 4 groups of when normalized by kilogram of body weight rats (2 factor ANOVA; $\left.\mathrm{F}_{(1,49)}=0.01\right)$. Following this observation, the adrenal weights of the PS and CON rats were averaged within the CMS and nonCMS groups and compared by Student's t-test. No statistical differences were found with this analysis $(\mathrm{t}=0.32, \mathrm{p}<0.56)$. 
Table 3.

\begin{tabular}{|c|c|}
\hline Age Range (days) & Event \\
\hline 22 & Weaning \\
\hline $26-30$ & Defensive Withdrawal Testing \\
\hline $30-34$ & Open Field Testing \\
\hline $39-43$ & Defensive Withdrawal Testing \\
\hline $59-63$ & FPS Testing (SDI Equip.) \\
\hline $66-70$ & 8-OH-DPAT and Startle Response \\
\hline $74-78$ & WAY100635 and Startle Response \\
\hline $90-94$ & ASR; Sucrose Assay \#1 \\
\hline $94-98$ & FPS Testing (MA Equip.); Sucrose Assay \#2 \\
\hline $103-107$ & Variable dB ASR; Sucrose Assay \#3 \\
\hline $111-115$ & Sucrose Assay \#4 \\
\hline $114-118$ & Sacrifice \\
\hline
\end{tabular}

Table 3 shows the time course and nature of the behavioral testing in which the CMS rats participated. Experimental use began at about post-natal age day 28 and continued at regular intervals until about post-natal age day 116. 
A. Nighttime Intake

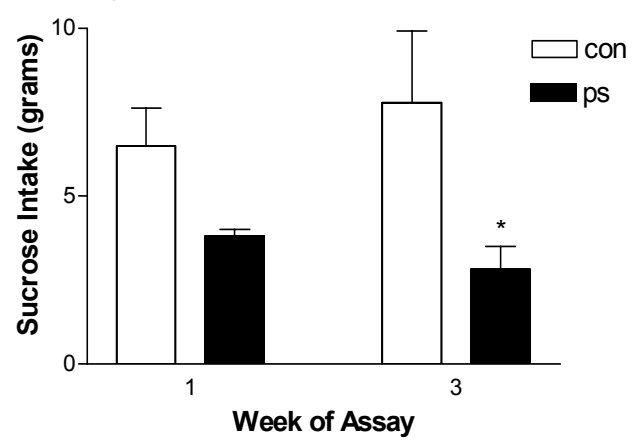

C. Water Intake

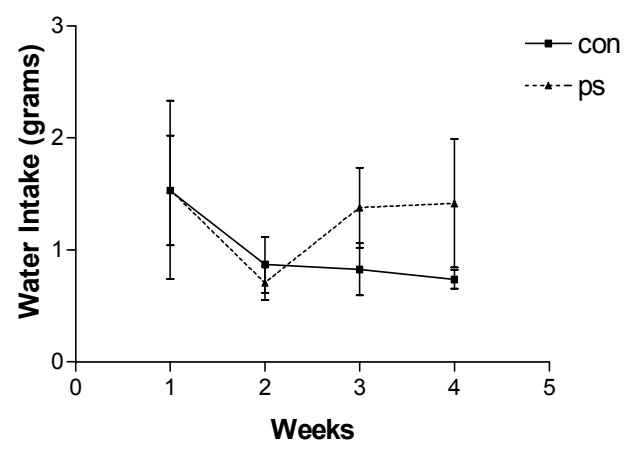

B. Daytime Intake

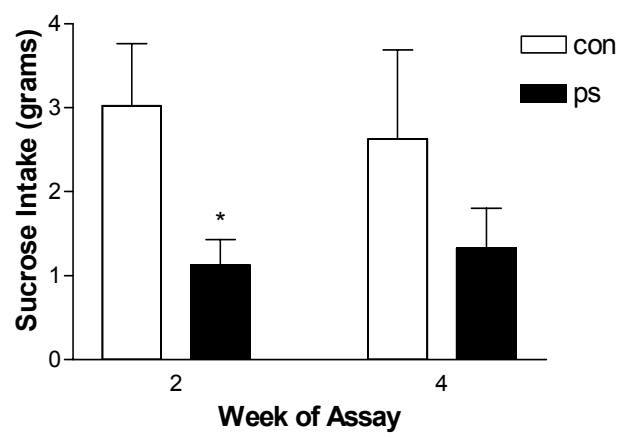

D. Body Weight

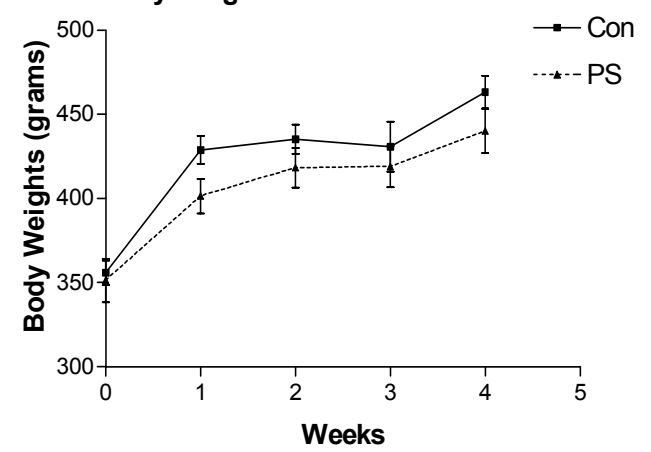

Figure 9: Comparison of sucrose intake, water intake and body weights over a 4 week period. A) Nighttime Sucrose intake was measured once during Weeks 1 and 3 and was generally larger in both groups than daytime intake. At nighttime, the PS group had less sucrose intake than the CON group. B) Daytime sucrose intake was measured once during weeks 2 and 4 . The PS group had significantly less intake during the daytime than the CON group . C) Water intake was comparable across both groups at night and during the day. No statistical differences were detected between the groups. D) Body weights were consistently lower in the PS group over the time week period of the assays. The weights shown at week zero were obtained 4 weeks prior to the start of measuring sucrose intake. The $\mathrm{n}$ values are 10 per group, representing 5 independent litters per group, except for week 1 , where the $n$ value was 5 to 6 , representing 2-3 independent litters. The reported values are means \pm S.E.M. (See results for statistical details; $* \mathrm{p}<0.05$ ) 
Table 4.

\begin{tabular}{|c|c|c|}
\hline Parameter & CON & PS \\
\hline CMS Adrenal Weight $(\mathrm{g} / \mathrm{Kg})$ & $0.1187 \pm 0.008$ & $0.1188 \pm 0.005$ \\
\hline Non-CMS Adrenal Weight $(\mathrm{g} / \mathrm{Kg})$ & $0.1130 \pm 0.005$ & $0.1137 \pm 0.007$ \\
\hline
\end{tabular}

Table 4 shows total adrenal weight normalized by kilogram of body weight. The CMS adrenal glands were taken from PS and CON exposed to numerous experimental procedures. The non-CMS adrenal glands were taken from age-matched naïve animals. No differences were detected in the data. Values are weights \pm S.E.M. (See results for details). 
Part C, Acoustic Startle and the Serotonergic Neurotransmitter System

Naïve CON and PS rats were randomly exposed to noise bursts over a range of nine different decibel levels within the same test session (Figure 10). The nine different decibel levels were presented within each of nine consecutive blocks of the test session. For simplicity of analysis, the data were compared at 90,95,100,115, 120 and 125 decibels and not across all decibels presented to the rats. Peak startle responses increased with increasing decibel levels but no differences were detected between the groups (RM 2 Factor ANOVA; $\mathrm{F}_{(1,23)}=0.49$ for group $\mathrm{F}_{(5,120)}=98.75, \mathrm{p}<0.0001$ for decibel level). The peak responses were also evaluated as a function of the presentation blocks. There was a decline in responding, but no differences were detected between the groups when block 1 was comapred to block 9 (see inset Figure 10; $\mathrm{F}_{(1,23)}=0.82$ for group; $\mathrm{F}_{(1,24)}=18.87$ for block, $\mathrm{p}<0.01$ ). Latency to peak startle response was also evaluated in this same study and no differences were detected with regard to group or decibel (RM 2 factor ANOVA; $F_{(1,23)}$ $=0.02$ for group $\mathrm{F}_{(5,120)}=0.53$ for decibel level) .

PS and CON rats were treated with two serotonergic drugs in order to determine if the lack of an increase in startle responding seen in PS rats with a high dose of yohimbine, as observed by White and Birkle (2001), was due to differences in sensitivity to $5-\mathrm{HT}_{1 \mathrm{~A}}$ receptor activation (Figure 11). Using the 5- $\mathrm{HT}_{1 \mathrm{~A}}$ receptor agonist, 8-OH-DPAT, PS and $\mathrm{CON}$ rats were exposed to 120 noise bursts on a 30 second inter-trial interval. A repeated measures design was incorporated such that each rat received each dose in a randomized fashion over the course of the study. There was a dose related increase in startle responding but no differences were detected between the groups (RM 2 factor ANOVA; $F_{(3,87)}=12.01, p<0.0001$ for dose, $F_{(1,87)}=1.46$ for group). Post hoc analysis with Fisher's 
LSD protected t-test revealed that the highest dose of 8-OH-DPAT increased responding in both groups above their own vehicle responses. Additionally, the post-hoc analysis showed that the responding of the PS rats at the $8 \mathrm{mg} / \mathrm{kg}$ dose was greater than that of the CON rats at the same dose. The data presented in Figure 11, part A are taken from the first half of the one hour test session. Time course analysis (data not shown) showed that the effect of the $8 \mathrm{mg} / \mathrm{kg}$ dose of $8-\mathrm{OH}-\mathrm{DPAT}$ was greatest during this time in the PS and CON rats.

The 5- $\mathrm{HT}_{1 \mathrm{~A}}$ antagonist, WAY-100635, was tested in the same fashion described for 8-OH-DPAT. WAY-100635 did not have a statistically significant effect on startle responding in either group (RM 2 factor ANOVA; $F_{(2,35)}=1.18$ for dose, $F_{(1,35)}=3.27$ for group). The data presented in Figure 11, Part B are taken from the second half of the test because time course analysis showed that the variability of peak startle responding was less during this time period (since there was no drug effect).

The latency to peak startle response was also evaluated in these two drug studies (Figure 12). Statistical analysis of the latencies for 8-OH-DPAT detected an effect of group and dose $\left(\mathrm{RM} 2\right.$ factor ANOVA; $\mathrm{F}_{(1,95)}=11.7, \mathrm{p}=0.0024$ for group, $\mathrm{F}_{(3,95)}=3.7$ for dose, $p=0.0162$ ). Furthermore, there was a significant interaction detected between the groups and dose suggesting that the groups were reacting differently to the drug (RM 2 factor ANOVA; $\left.\mathrm{F}_{(66,95)}=9.6, \mathrm{p}<0.0001\right)$. Post-hoc analysis with Fisher's LSD protected t-test revealed that the PS rats had increasing latencies with increasing doses. In contrast, the latencies in the CON rats did not reveal a consistent change with increasing doses. Statistical analysis of the latencies to peak startle response were evaluated for WAY100635 as well (Figure 12). The analysis did not detect any differences between the groups 
or with the various doses (RM 2 factor ANOVA; $F_{(1,24)}=0.01$ for group, $F_{(2,24)}=0.01$ for dose).

The latencies to peak response in the CON group measured in response to the vehicle injection (and the two doses of WAY-100635) were lower than those reported for the CON rats in the 8-OH-DPAT study (Figure 12). The latencies in the $\mathrm{CON}$ rats for the vehicle doses in both studies were compared by Student's t-tests and not found to be different $(\mathrm{t}=2.24, \mathrm{p}<0.06)$. 


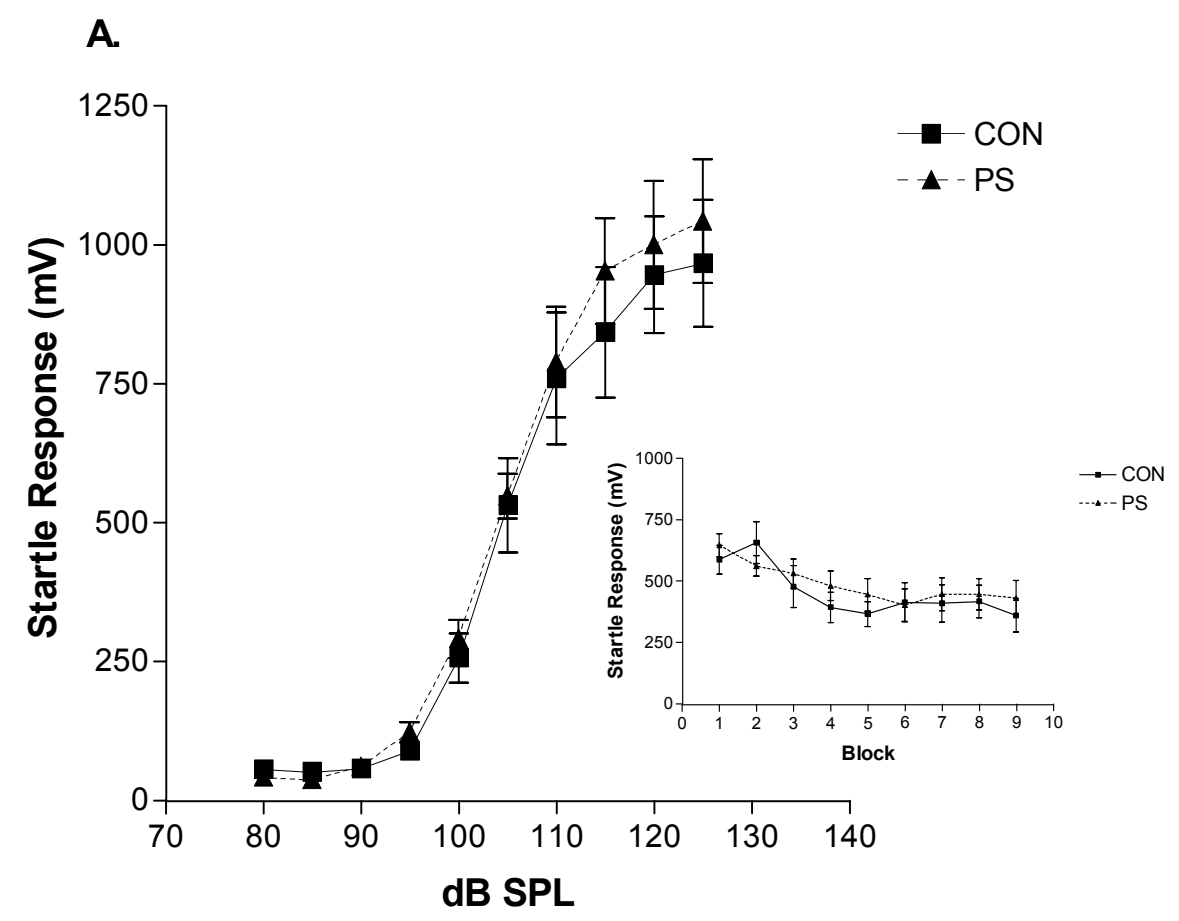

B.

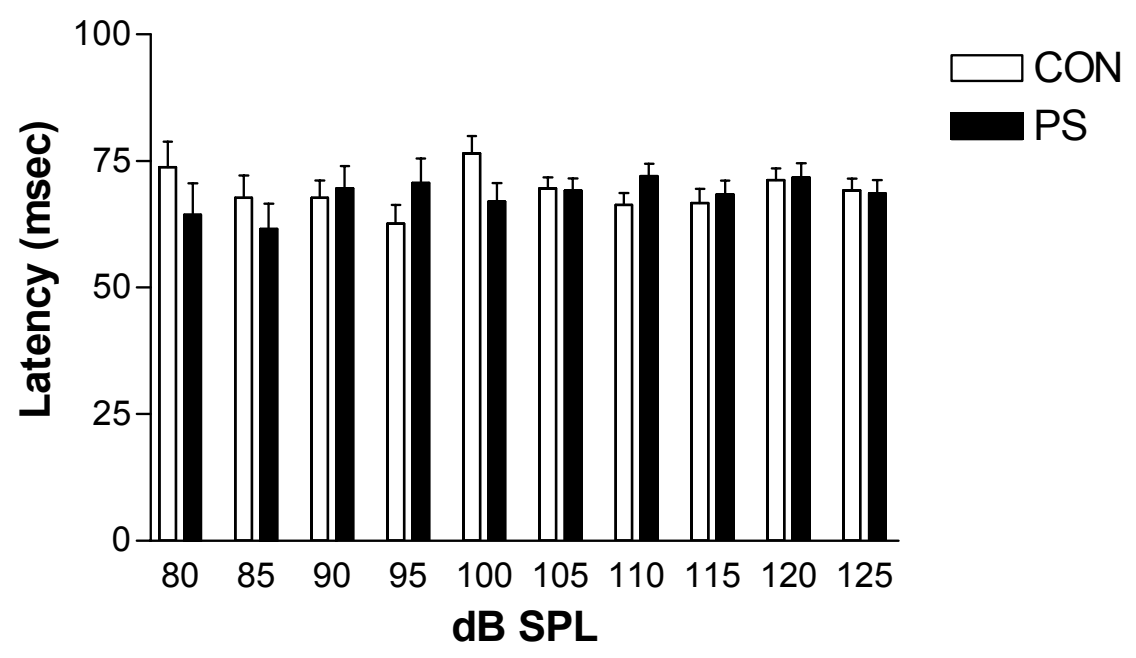

Figure 10. Acoustic startle responding to noise bursts covering a range of decibel levels presented within the same test session (9 presentations per decibel level). A) Peak startle responses increased with increasing decibel levels, but CON and PS rats were not different in their responses at any of the decibel levels. The inset shows the general decline in responding to the same decibel levels as the session progressed. B) Latency to reach the peak startle response was not different at any decibel level or between the groups. There were 12 rats per group representing 6 independent litters per group. The experiment was conducted in MA Equipment. (See results for details) 


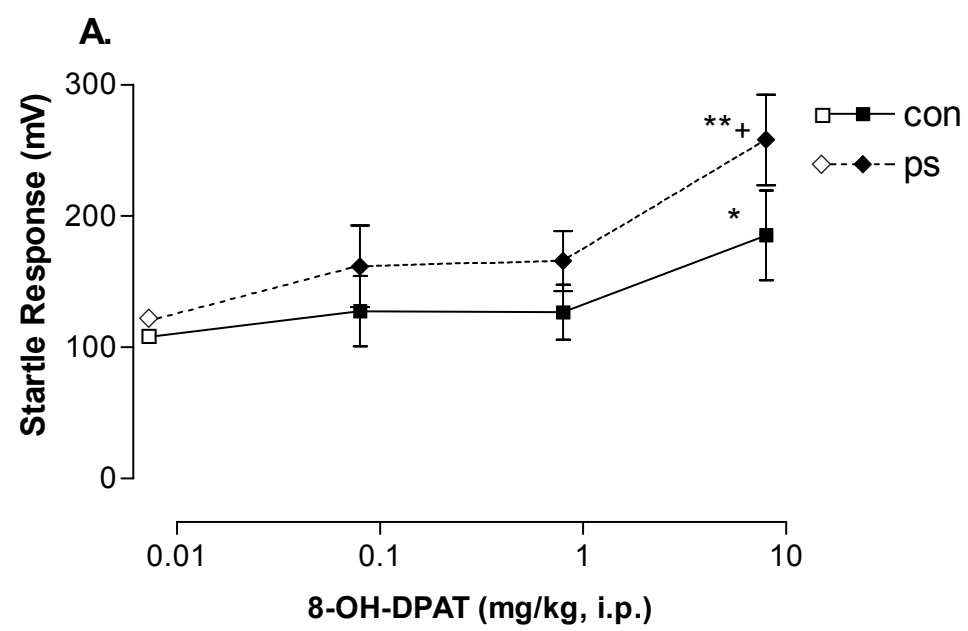

B.

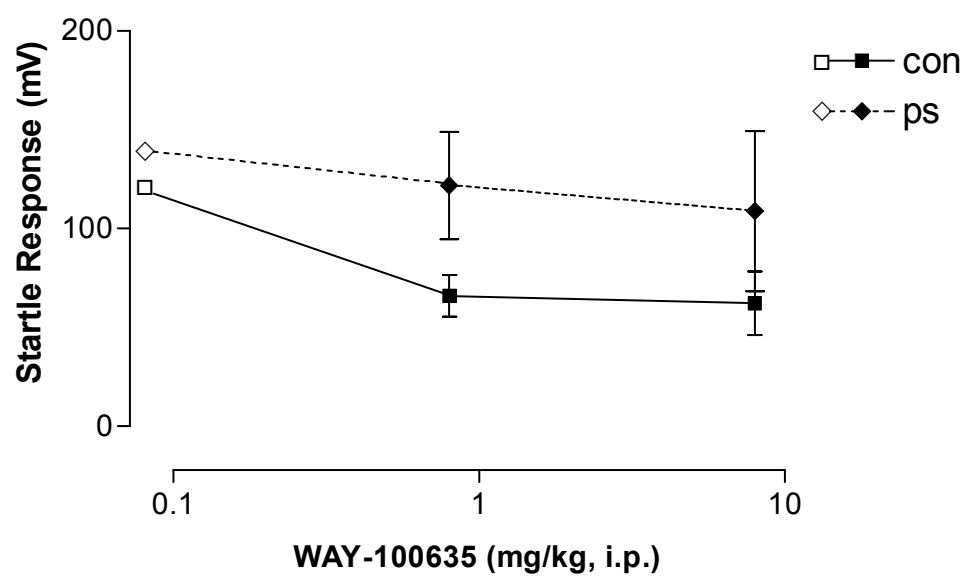

Figure 11: Peak startle measurements in the presence of drugs selective for the $5-\mathrm{HT}_{1 \mathrm{~A}}$ receptor. A) Startle responses are increased in the presence of the $5-\mathrm{HT}_{1 \mathrm{~A}}$ agonist, 8-OH-DPAT, but only in the PS group. There were 11 rats per group, representing 6 independent litters per group in this study. B) Startle responses are unchanged in the presence of the $5-\mathrm{HT}_{1 \mathrm{~A}}$ antagonist, WAY-100635. No differences were detected between or within the groups with WAY-100635. There were 6 rats per group, representing 3 independent litters per group in this study. In parts A \& B, the responses to the vehicle are indicated next to the y-axis with open symbols.

Experiments were conducted in MA equipment. Values are peak startle amplitudes \pm S.E.M. (See results for statistical details; ${ }^{* *} \mathrm{p}<0.01$ within group compared to vehicle and the other doses, ${ }^{*} \mathrm{p}<0.05$ within group compared to vehicle; $+\mathrm{p}<0.01$ between groups same dose). 

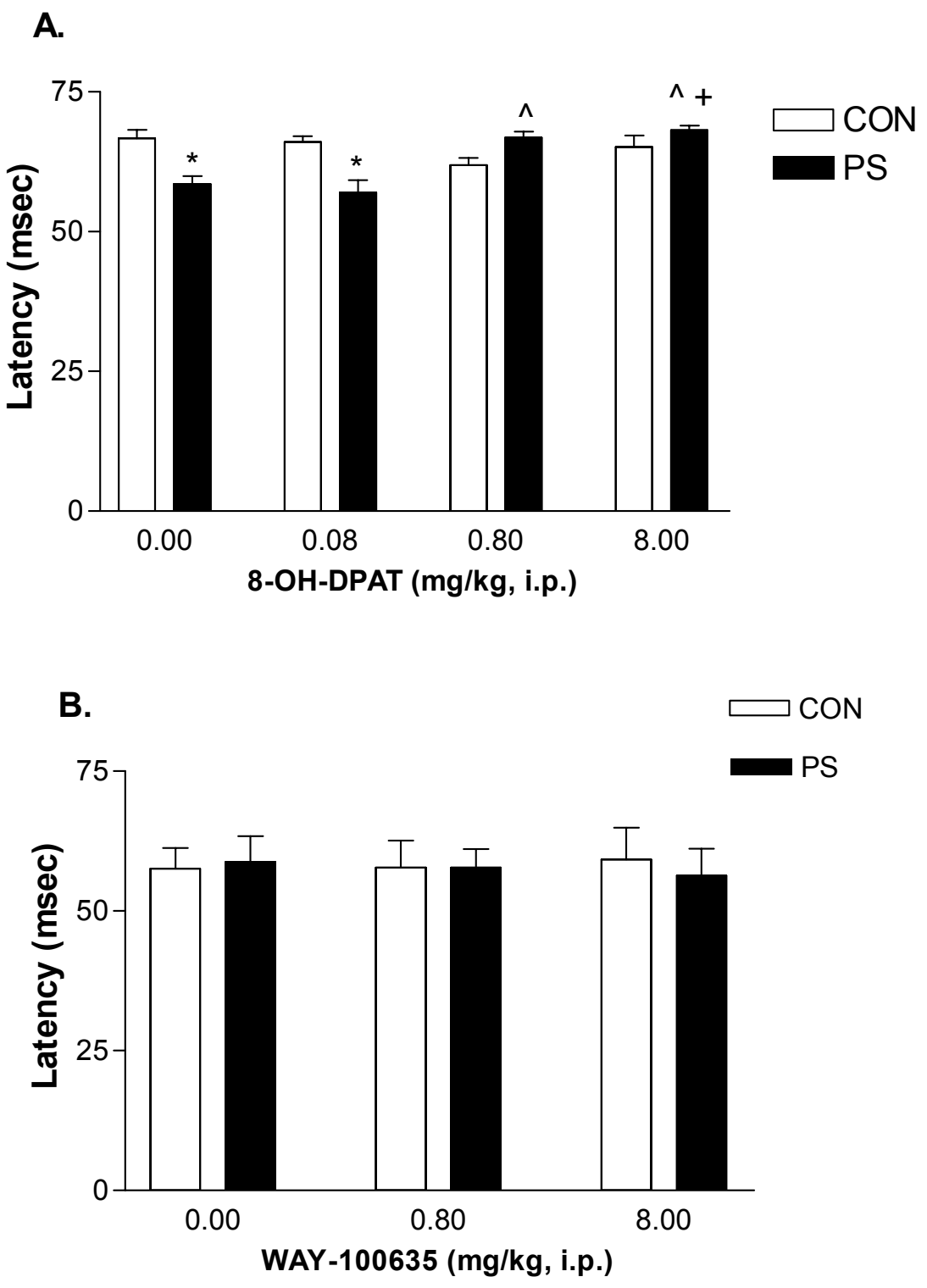

Figure 12. The mean latency to the peak startle responses shown in Figure X for 8-OH-DPAT and WAY-100635. A) Latencies for the 8-OH-DPAT treated rats. The PS rats show a dose related increase in latencies to reach the peak startle response. The CON rats are not do show a dose related change. B) Latencies for the WAY-100635 treated rats where not different with respect to dose or group. The values reported are milliseconds \pm S.E.M. (see results for details; * $\mathrm{p}<0.05$ between groups same dose and vehicle; $\wedge \mathrm{p}<0.05$ within group compared to 0.08 dose; $+\mathrm{p}<0.01$ within group compared to vehicle) 


\section{Part D, Conditioned Fear Assessments}

Fear-potentiated startle testing was performed on adult PS and CON rats and two parameters were evaluated, peak response and null period average responding in this initial set of experiments. The first set of experiments were conducted such that the training context and testing context were exactly the same. The two variables that were manipulated in these experiments were the shock intensity used in training and the number of training days. Figure 13 shows the percent difference scores, calculated from the peak startle response values obtained in these experiments, were not different from zero. In fact, at the highest shock intensity used, the percent difference scores were actually negative.

Despite the calculation of negative difference scores, absolute peak values following FPS training were increased during the testing trials (Figure 14). After 2 days of training at a shock intensity of $0.5 \mathrm{~mA}$, no differences were detected between the groups in terms of elevated peak responses ( Part A, Figure 14). However, startle responding was generally increased during the testing trials in both groups above that of the leader trials $\left(\mathrm{RM} 2\right.$ factor ANOVA; $\mathrm{F}_{(1,65)}=0.04$ for group and $\mathrm{F}_{(2,65)}=6.35, \mathrm{p}=0.0004$ for trial type). Post-hoc analysis confirmed that the responding during the testing trials was increased above that of the leader trials in both groups. After 4 days of training at $0.5 \mathrm{~mA}$ shock intensity, there was not an effect of group but there was an effect of training (Part B, Figure 14; RM 2 factor ANOVA; $\mathrm{F}_{(1,44)}=2.42$ for group, $\mathrm{F}_{(2,44)}=4.31, \mathrm{p}=0.0242$ for training). Post hoc analysis with Fisher's LSD protected t-tests revealed that only the PS group had a large increase in peak startle responses testing trials compared to the leader trials and baseline testing. Interestingly, the responses to the testing trials from the CON 
group did not show a statistically significant elevation in startle responding above the leader trials or even compared to their own baseline results.

The null period average responses were also considered from the same set of experiments just described (see methods for a description of this parameter). Null period average responses were evaluated by trial type (i.e. noise alone versus light plus noise) and no differences were found (data not shown; RM 2 factor ANOVA; $F_{(1,20)}=2.2$ for group and $\mathrm{F}_{(1,20)}=0.05$ for trial type). Figure 15 shows the null period responses from baseline ASR testing trials, leader trials and testing trials after two and four days of training (Parts A $\&$ B, respectively). The PS rats, after two training days, had a greater depression in null period responding during the leader trials and testing trials when compared to their baseline values and the CON group (RM 2 factor ANOVA; $F_{(1,68)}=23.46, p<0.0001$ for group, $\mathrm{F}_{(2,68)}=9.80, \mathrm{p}=0.0003$ for session). The statistical analysis revealed a significant interaction between the groups and the trial types suggesting that the two groups were responding differently to the test session $\left(\mathrm{RM} 2\right.$ factor ANOVA; $\mathrm{F}_{(42,68)}=5.83, \mathrm{p}=$ 0.0058). In fact, post hoc analyses with Fisher's LSD protected t-tests demonstrated that the CON group's null period responding was increased during the testing trials versus their leader trial null period responding. After 4 days of training, PS rats continue to show markedly depressed null period responding during both halves of the test; however, the training effect was lost $\left(\mathrm{RM} 2\right.$ factor ANOVA; $\mathrm{F}_{(1,44)}=17.55, \mathrm{p}=0.0011$ for group, $\mathrm{F}_{(2,44)}$ $=1.4$ for training). Again, there was an interaction with group and session suggesting that the two groups were reacting differently during the test and may have contributed to the loss of the training effect $\left(\mathrm{RM} 2\right.$ factor ANOVA; $\left.\mathrm{F}_{(2,65)}=9.90, \mathrm{p}=0.0006\right)$. Indeed, the 
CON rats, after 4 days of training had increased null period responding during the testing trials as determined by post hoc analysis with Fisher's LSD protected t-test.

Observationally based conditioned freezing experiments were conducted and seconds freezing per minute and rears per minute were compared between the groups (Figure 16). There was an effect of group and session detected in the amount of time spent freezing ( $\mathrm{RM} 2$ factor ANOVA; $\mathrm{F}_{(1,59)}=5.45, \mathrm{p}=0.0313$ for group, $\mathrm{F}_{(2,59)}=86.00$, $\mathrm{p}<0.0001$ for session). Furthermore, there was an interaction detected with regard to group and session $\left(\mathrm{RM} 2\right.$ factor ANOVA; $\left.\mathrm{F}_{(36,59)}=3.38, \mathrm{p}=0.0452\right)$. Post-hoc analyses with Fisher's LSD protected t-tests revealed that the PS rats were freezing more to the acute footshock as well as 24 hours later in the same context without the footshock. Rears per minute were also affected as a function of shock, but not as a function of group (RM 2 factor ANOVA; $\mathrm{F}_{(1,59)}=0.30$ for group, $\mathrm{F}_{(1,59)}=65.07, \mathrm{p}<0.0001$ for shock). Post-hoc analysis revealed that rears were markedly decreased after the shock as well as the next day. Rears were increased the next day in the CON group compared to the post-shock period, but this was not found to be true for the PS group.

The PS and CON rats were also evaluated for their ability to freeze to an explicit cue, a light (Figure 17). Freezing to the light was non-existent to either the context or the light itself during the training period for both groups but increased dramatically the next day during the testing session (RM 2 factor ANOVA: $\mathrm{F}_{(3,71)}=71.74, \mathrm{p}<0.0001$ for training). Post-hoc analysis demonstrated that there was freezing to the context but there was more freezing to the light cue. However, no differences were found between the groups $\left(\mathrm{RM} 2\right.$ factor ANOVA; $\left.\mathrm{F}_{(1,71)}=0.17\right)$. Rears were evaluated in the same fashion as freezing and were found to be decreased following the training session, but no differences 
were found between the group or between context and the light cue (RM 2 factor ANOVA; $\mathrm{F}_{(1,71)}=0.08$ for group, $\mathrm{F}_{(3,71)}=25.81$ for training effect, $\left.\mathrm{p}<0.0001\right)$.

Another FPS experiment was conducted with slight methodological changes aimed at altering the context (the shock grids were not in place during testing and $70 \% \mathrm{EtOH}$ was used to clean between rats) (Figure 18). Statistical analysis of the leader trials and testing trials session data did not reveal an effect of group but demonstrated an effect of training $\left(\mathrm{RM} 2\right.$ factor ANOVA; $\mathrm{F}_{(1,55)}=3.97$ for group and $\mathrm{F}_{(2,55)}=27.39, \mathrm{p}<0.0001$ for trial type). Additionally, the statistical analysis revealed an interaction with group and trial type, indicating that the two groups were responding differently to the light + noise trials ( $\mathrm{F}$ $(26,55)=4.56, \mathrm{p}=0.0422)$. Post hoc analysis with Fisher's LSD protected t-test revealed that responding to the light + noise trials in the CON groups was elevated above their noise alone as well as the light + noise trials of the PS group. Difference scores were calculated by simply subtracting noise alone responses from light + noise responses and the difference scores for each group were compared to zero and each other. Both were found to be significantly different from zero (Student's $\mathrm{t}$-test; $\mathrm{t}=4.01, \mathrm{p}=0.0015$ for $\mathrm{CON}$ and $\mathrm{t}=$ $5.90, \mathrm{p}=0.0001$ for PS) but not from each other (Student's $\mathrm{t}$-test; $\mathrm{t}=0.37$ ).

Difference scores were evaluated as a function of repeated CS exposure within the FPS test session shown in Figure 18 (Part B, Figure 18). The testing trials of the test session were divided into 3 sequential blocks of 10 trials each with the blocks composed of 5 noise alone trials and 5 light plus noise trials. Difference scores were calculated using the peak startle responses within these blocks. A one-factor repeated measures analysis for each group detected an effect of block for each group (RM 1 factor ANOVA; $F_{(2,41)}=5.82$, $\mathrm{p}=0.0082$ for $\mathrm{CON}$ and $\mathrm{F}_{(2,41)}=3.42, \mathrm{p}=0.0476$ for PS). Post hoc anlaysis showed that, 
in both groups, block 3 was less than block 1 . Finally, the difference scores of block one were compared between the groups, but were found not to be different (Student's $\mathrm{t}$-test; $\mathrm{t}=$ $1.68, \mathrm{p}=0.1042)$.

Figure 19 also shows the null period responses from the same experiment. Statistical analysis of this data set revealed an effect of the training but not of group (RM 2 factor ANOVA; $\mathrm{F}_{(2,83)}=14.61, \mathrm{p}<0.0001$ for session, $\mathrm{F}_{(1,83)}=0.99$ for group). Post hoc analysis using Fisher's LSD protected t-test demonstrated that testing trial null period responding was decreased in both groups, but only during the testing trials and not during the leader trials.

Shock induced movements across both training sessions were evaluated for some of the rats in this last FPS experiment. The average peak movement response on day 1 of training was $552.2 \pm 76 \mathrm{mV}$ for PS rats and $517.1 \pm 56 \mathrm{mV}$ for $\mathrm{CON}$ rats, respectively. On day 2, the shock induced movement response was $623.1 \pm 97 \mathrm{mV}$ for the PS rats and 668.2 $\pm 107 \mathrm{mV}$ for the CON rats. Statistical analysis did not detect any differences in these parameters with respect to group but differences were found with regard to day of training (RM 2 factor ANOVA; $\mathrm{F}_{(1,39)}=0.01$ for group, $\mathrm{F}_{(1,83)}=6.25$ for day, $\mathrm{p}=0.0223$ ). Post-hoc analysis with Fisher's LSD test showed that responses in the CON group were elevated the second day of training.

Latencies to peak startle response were evaluated in the FPS experiments shown in Figure 15, part A and in Figure 18 (Table 5). First, latencies during the testing trials of the experiment shown in Figure 18 were analyzed by trial type according to group (i.e. noise alone or light + noise) but no differences were found ( $\mathrm{RM} 2$ factor ANOVA; $\mathrm{F}_{(1,20)}=2.2$ for group and $\mathrm{F}_{(1,20)}=0.05$ for trial type). Since no differences were found, trial types 
were averaged during the testing trials for further analyses of latency to peak startle (see Table 5). In the FPS experiment shown in Figure 15 (labeled "A" in Table 5), ANOVA detected an effect of group and training when the baseline trials, leader trials and testing trials were analyzed (RM 2 factor ANOVA; $\mathrm{F}_{(1,68)}=5.32, \mathrm{p}=0.0313$ for group and $\mathrm{F}_{(2,68)}$ $=119.84, \mathrm{p}<0.0001$ for the training effect). Post hoc analysis revealed that the PS rats reached peak startle times faster during the baseline test session and during the testing trials when compared to the $\mathrm{CON}$ rats. Both groups demonstrated a dramatic decrease in time to reach peak startle response following the FPS training, which was evident during the leader trials as well as the testing trials. Latency to peak responses were analyzed in the same way for the FPS experiment shown in Figure 18 (labeled "B" in Table 5). In this experiment, there was an effect of group and training (RM 2 factor ANOVA; $F_{(1,83)}=4.30$, $\mathrm{p}=0.0481$ for group and $\mathrm{F}_{(2,83)}=57.80, \mathrm{p}<0.0001$ for the training effect). Post-hoc analysis of this data set demonstrated that latencies to peak response were shorter in both groups during the leader trials and even shorter during the testing trials. Furthermore, the post-hoc analysis demonstrated that the PS rats had shorter times than the CON rats during the leader trials.

A final FPS experiment was conducted as described in the methods, including the contextual changes, except that the light and shock were intentionally not paired during the training sessions (data not shown). Baseline peak startle responding in the 8 naïve CON and PS rats used in this experiment were not different $(366 \pm 55 \mathrm{mV}$ and $394 \pm 51 \mathrm{mV}$ for CON and PS, respectively). Following two days of training, the calculated difference scores were negative indicating that, as expected, potentiation of startle had not occured. Interestingly, the pattern of peak startle responses resembled that shown in Figure 15, with 
the PS rats demonstrating an increase in peak startle responses to the testing trials, regardless of trial type (eerily, both groups had the same leader trial responses of $300 \pm 52$ $\mathrm{mV}$; whereas testing trial responses were $402 \pm 72 \mathrm{mV}$ for $\mathrm{CON}$ rats and $510 \pm 83 \mathrm{mV}$ for PS rats). However, statistical differences were not detected in this data set when baseline, leader trial and testing trial responses were evaluated $\left(\mathrm{RM} 2\right.$ factor ANOVA; $\mathrm{F}_{(1,47)}=1.61$ for group and $\mathrm{F}_{(2,47)}=2.49$ for training effect). 


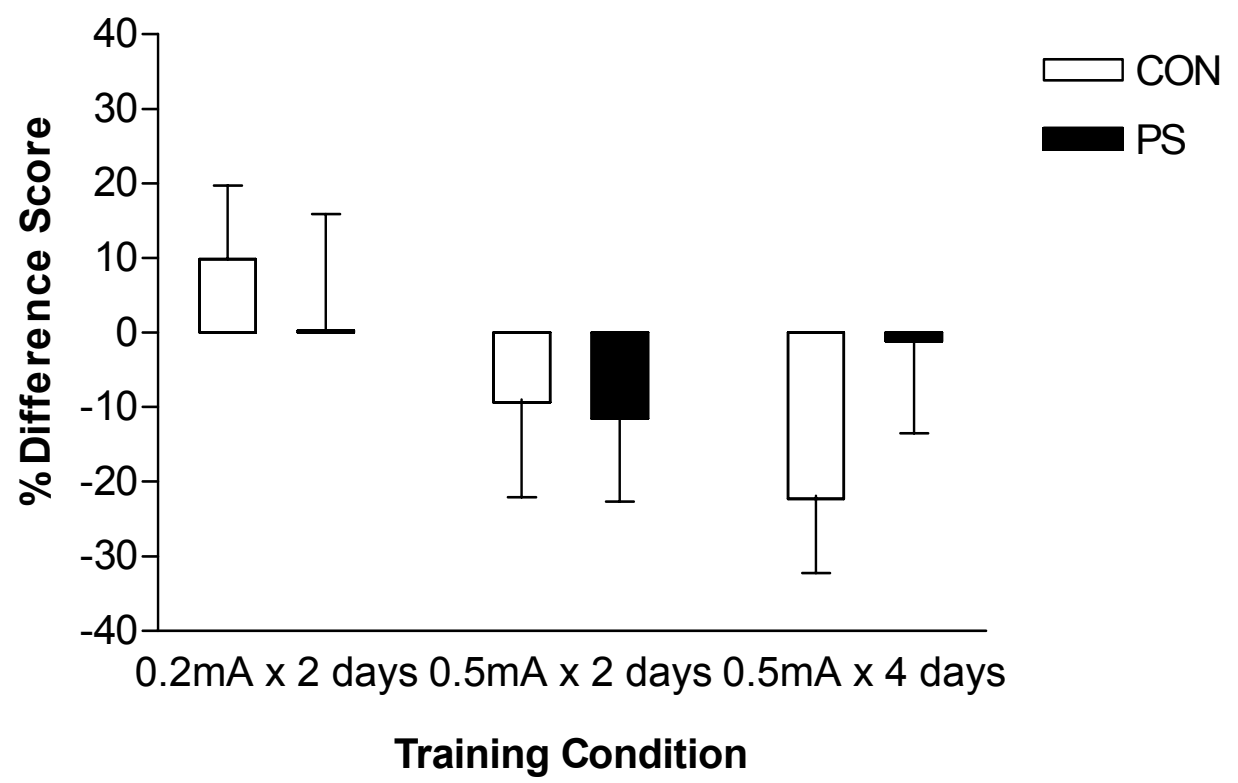

Figure 13. The percent difference scores from several experiments utilizing different training conditions and tested in the same context. No differences were detected between the groups with any of the experimental conditions. All experiments were conducted in SDI Equipment. \% Difference $=($ mean light plus noise trials - mean noise alone)/mean noise alone X 100. Values are means \pm S.E.M. (See results for statistical details). 
A. 2 training days

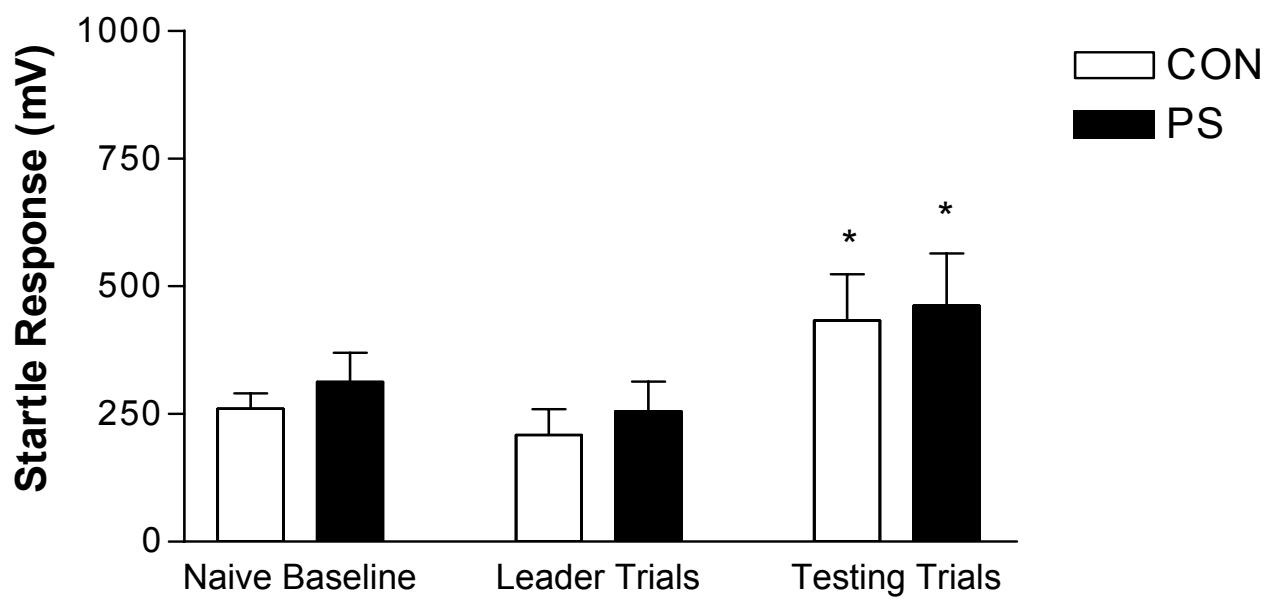

B. 4 training Days

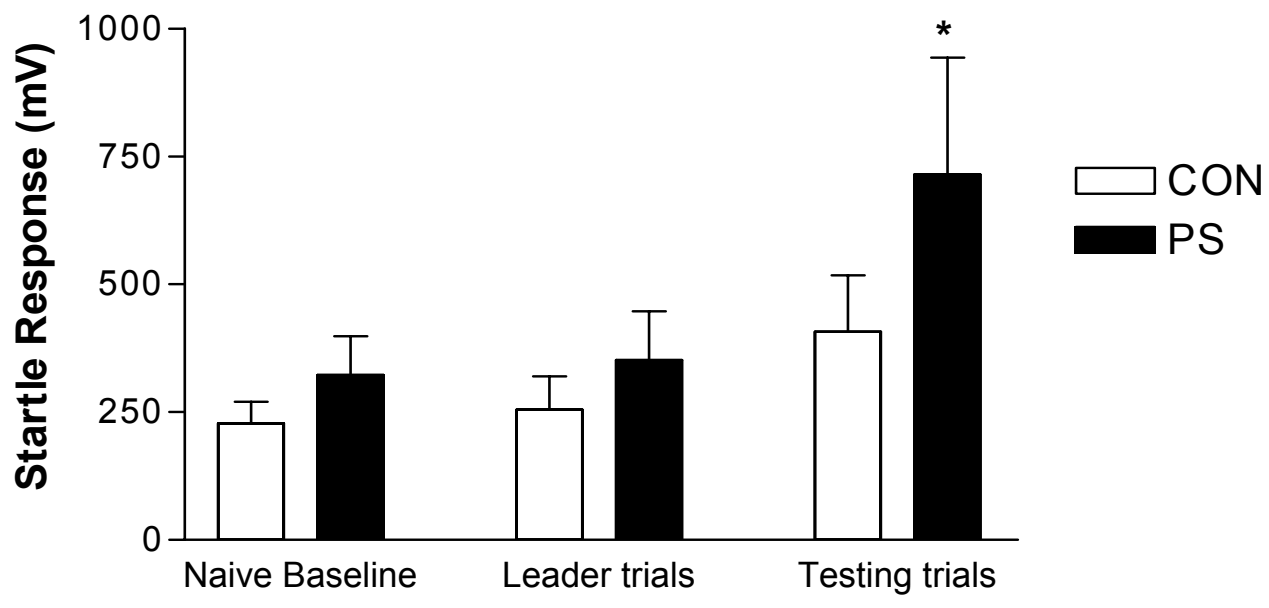

Figure 14. A comparison of startle responses before and after FPS training at 0.5 $\mathrm{mA}$ shock intensity. Responses to both trial types were averaged for the testing trial results. A) Baseline responses were not different between the groups. Leader trial startle responding was not different from baseline responding in either group after 2 training days. During the testing trials both groups increased responding above baseline and first half responding. ( $\mathrm{n}=11-12$ per group). B) Baseline differences were not detected between the groups. Differences in startle responding were not detected within in the CON group after 4 days of training, although there is a non-significant trend towards increased responding during the testing trials. During the testing trials, the PS group showed increased responding above baseline and leader trial responding as well as testing trial responses of the CON group ( $\mathrm{n}=7-8$ per group). The experiments were conducted in SDI equipment. The reported values are peak amplitudes \pm S.E.M. (See results for statistical details; * $\mathrm{p}<0.05$ for within group leader/testing trial comparison). 
A. 2 Training Days

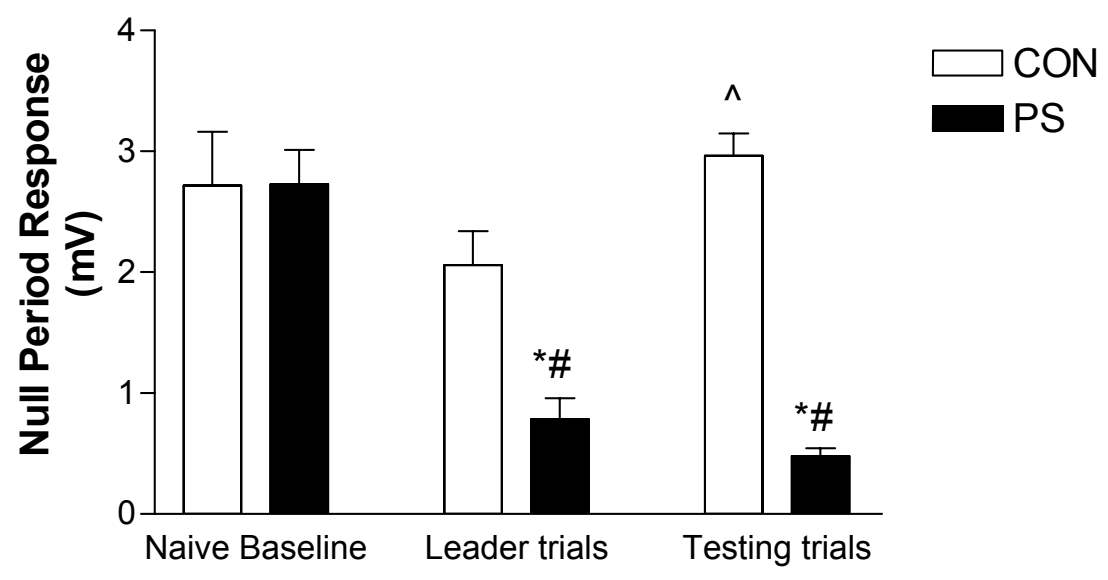

B. 4 Training Days

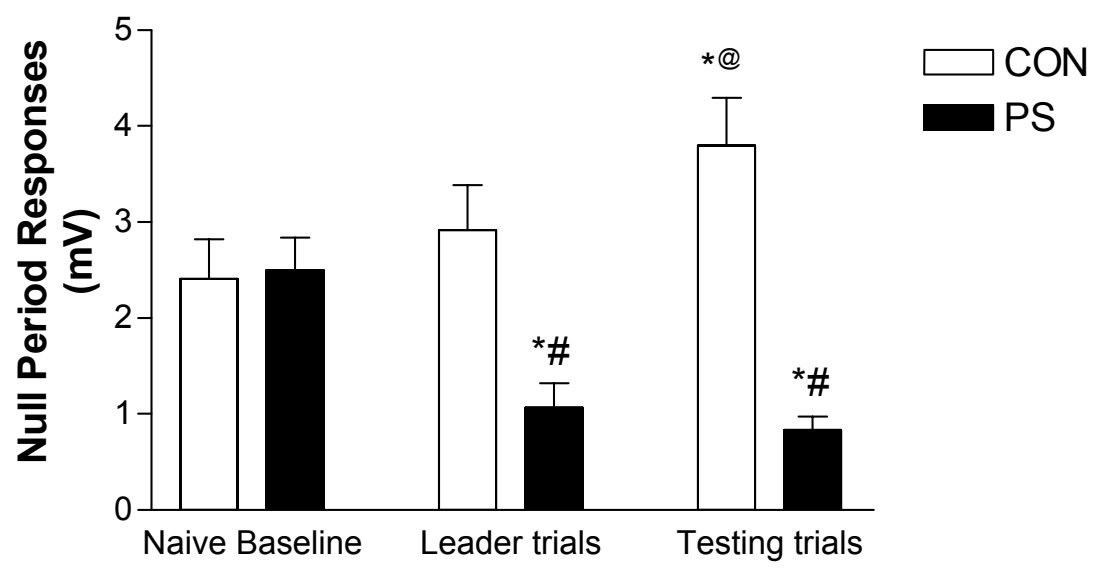

Figure 15. A comparison of null period responses in the same experiments that were shown in Figure 8. Responses to both trial types were averaged for the testing trials. A) After training for 2 days, both groups have depressed null period responses during the leader trials, but the difference is greatest in the PS group. PS null period responses remain depressed during the leader trials but the CON group shows increased null period responses. B) After training for 4 days, the PS group has depressed null period responding during the entire test session compared to its baseline value. However, after 4 training days, the CON group has increased null period responding during the testing trials. The values are means \pm S.E.M. (See results for statistical details; ${ }^{*} \mathrm{p}<0.01$ for within group baseline comparison; $\# \mathrm{p}<0.01$ for between group each session comparison; $@ \mathrm{p}<0.05$ for within group comparison of leader and testing trials; ${ }^{\wedge} \mathrm{p}<0.05$ for within group comparison of leader trials and testing trials). 


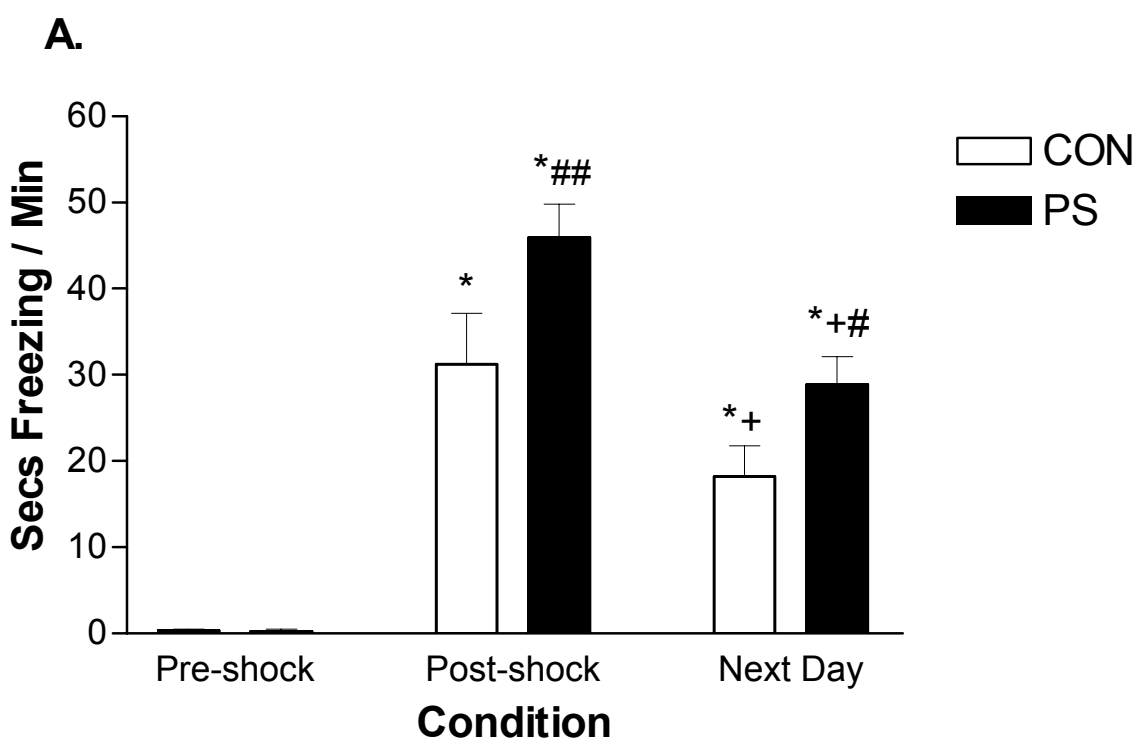

B.

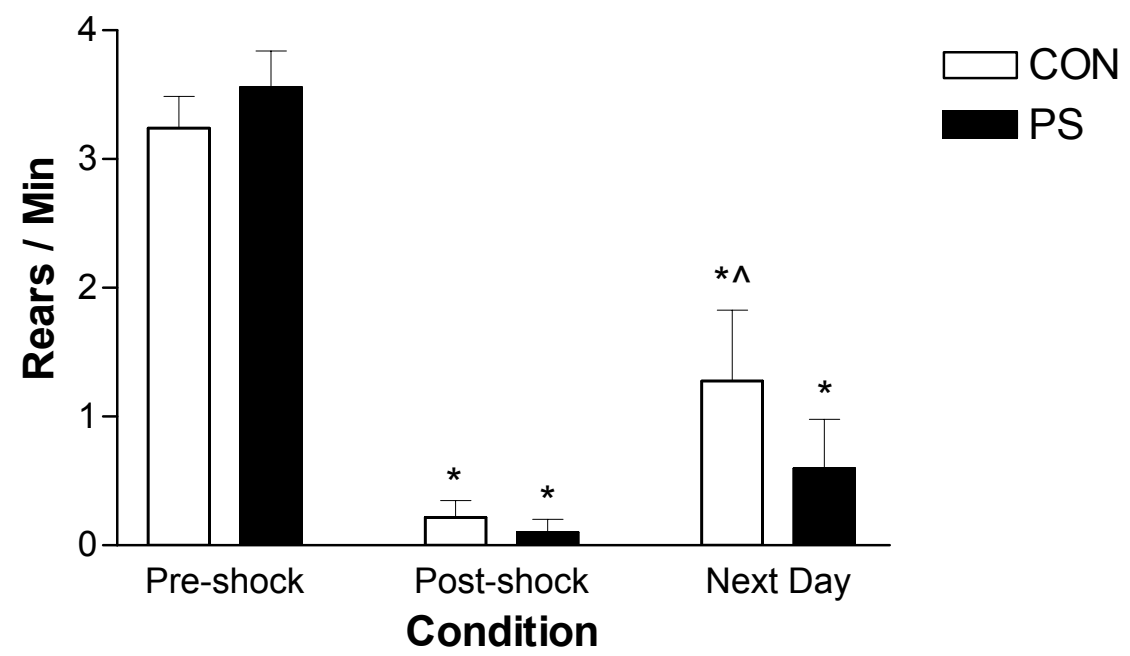

Figure 16: Behavioral inhibition during the conditioned freezing experiment. A) The seconds per minute spent freezing was negligible during the pre-shock period but increased in both groups following the onset of the first shock with the PS group showing more freezing to the acute shock presentation. Freezing was also evident the following day in both groups, with the PS group showing significantly more freezing in the context where shock was delivered. B ) Rears per minute were high in the pre-shock period in both groups and negligible in the acute post-shock period in both groups. Testing the following day showed that the rears were increasing only in the CON group. The $\mathrm{n}$ value was 10 per group. Values are means \pm S.E.M. (see results for statistical details; $\# \mathrm{p}<0.05$ and $\# \# \mathrm{p}<0.01$ for between group comparisons; $* \mathrm{p}<0.01$ for within group comparisons to the preshock condition; $+\mathrm{p}<0.01$ within group compared to the post-shock condition; ${ }^{\wedge} \mathrm{p}<0.05$ within group compared to the post-shock condition). 


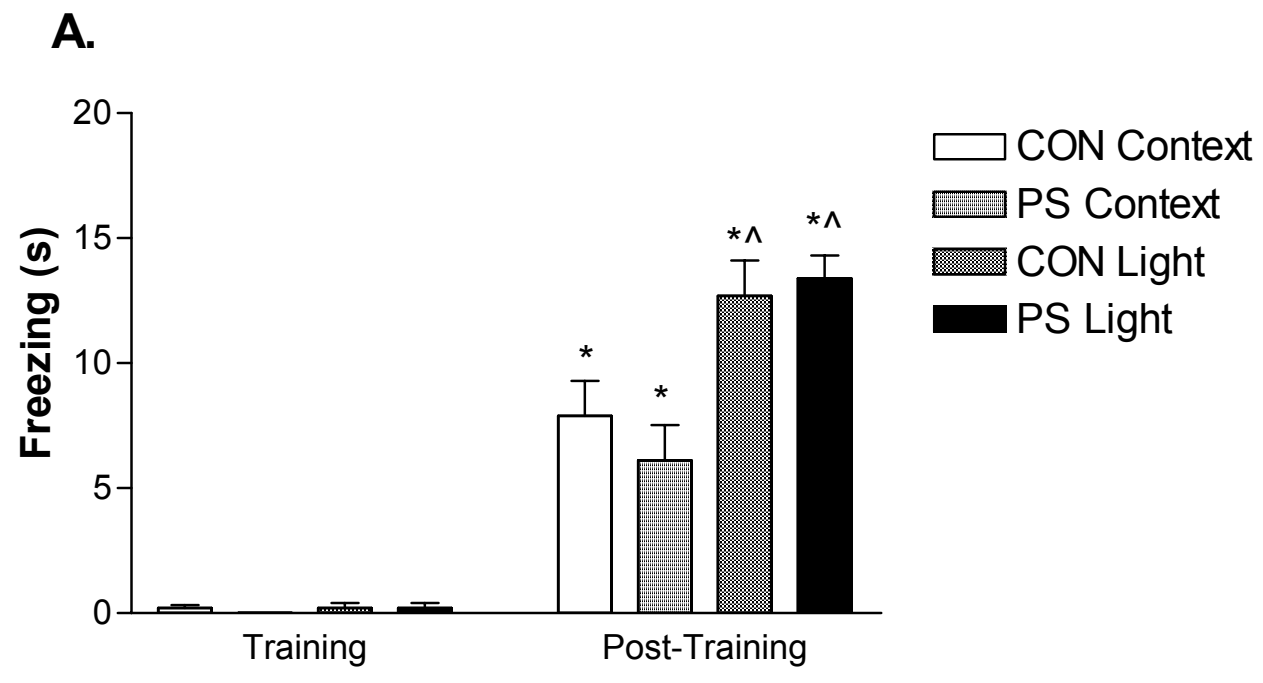

B.

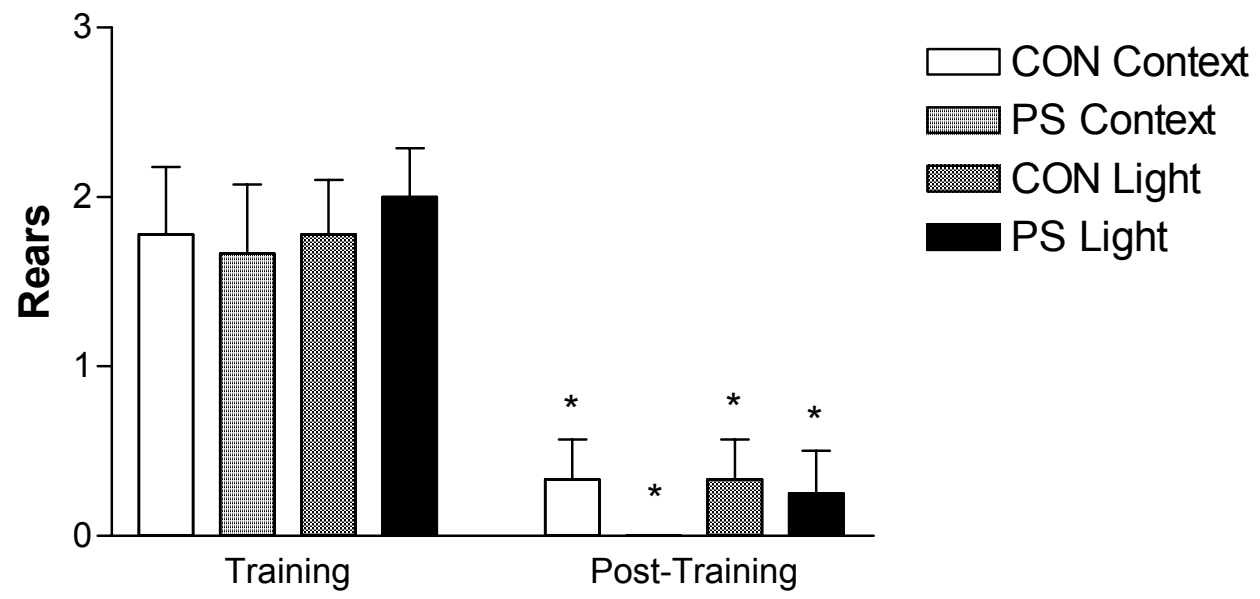

Figure 17. Behavior before and after conditioning to an explicit light cue. A) The freezing times reported in the graph were recorded for 20 seconds prior to the light (e.g. the context) and for the 20 seconds of exposure to the light (e.g. the light). Freezing was increased in both instances following the training. B) The number of rears counted during the same conditions as A. Rears were attenuated following the training. The data for the training session are from the first training trial. There were 9 animals per group representing 4-5 independent litters. The values are means \pm S.E.M. (see results for details; ${ }^{*} \mathrm{p}<0.01$ for training effect within group, ${ }^{\wedge} \mathrm{p}<0.01$ for effect of context versus light within group). 


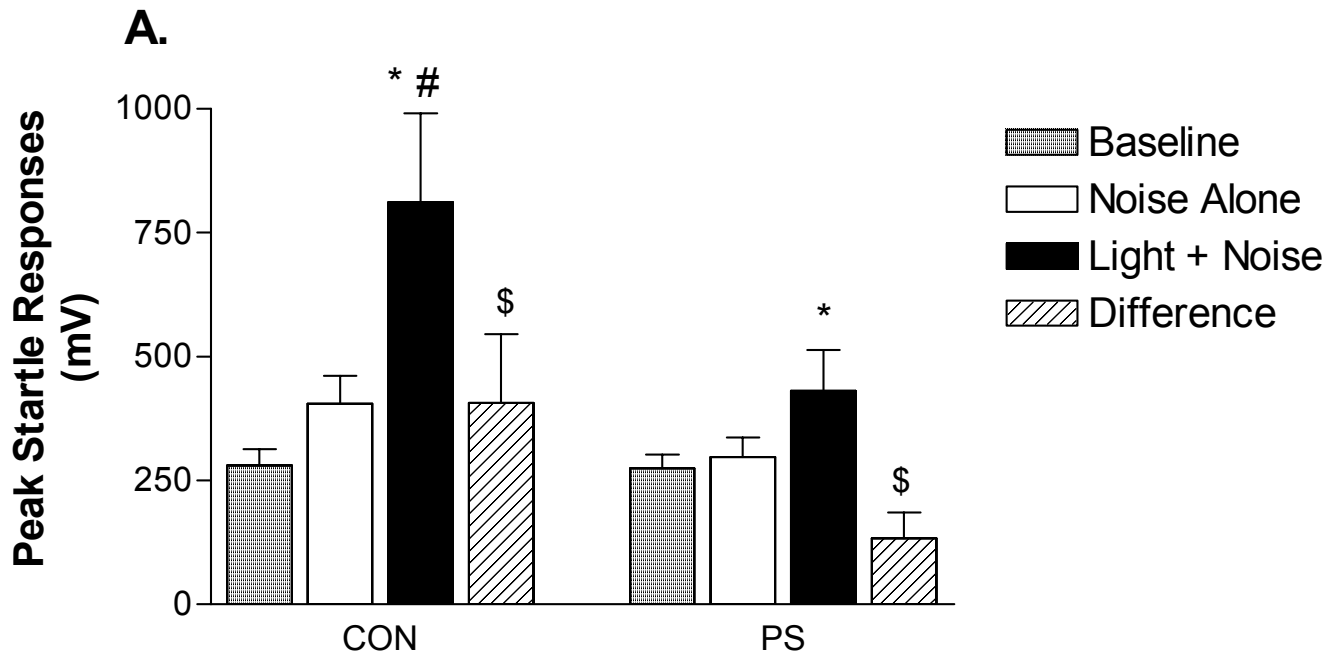

B.

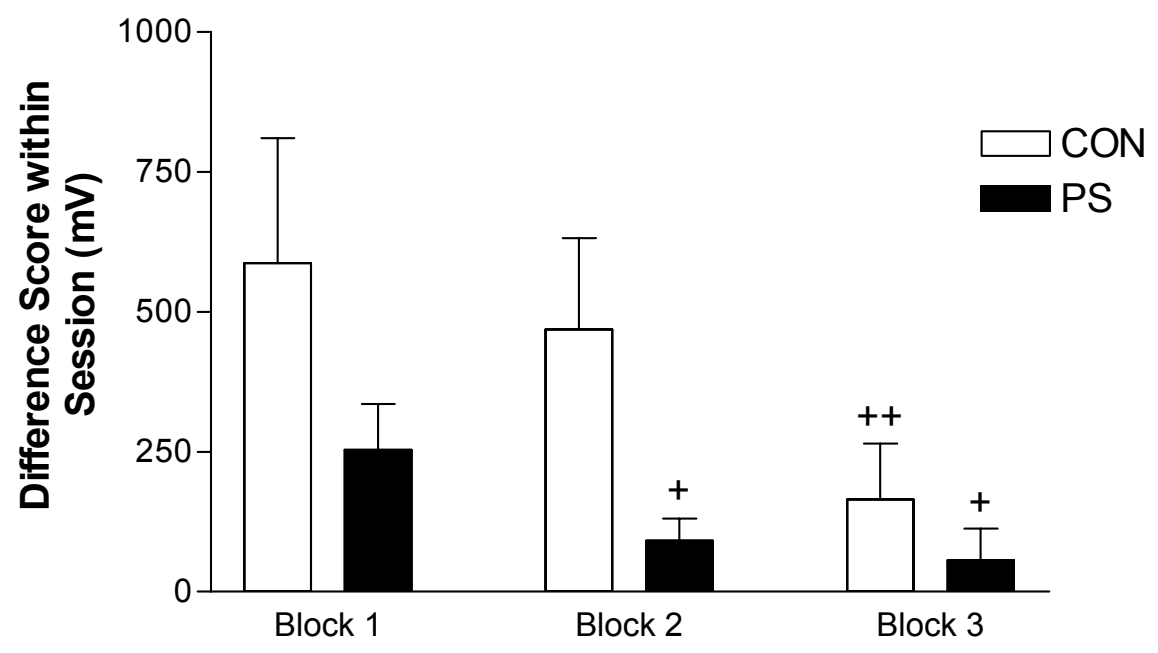

Figure 18. Peak startle responding from the fear-potentiated startle experiment. A) Baseline startle responses were not different between the two groups. Following training, the $\mathrm{CON}$ rats demonstrated a robust potentiation of startle; whereas, the PS rats only had a small potentiation. B) Difference scores were calculated within the test session and became smaller as the session progressed. This resembles a learning extinction phenomenon and demonstrates that both the CON and PS rats learned the CS-US association. The values reported are means \pm S.E.M. There were 14 rats per group representing 7 independent litters per group. The experiment was conducted in SDI equipment (See results statistical details; ${ }^{*} \mathrm{p}<0.01$ within group noise alone versus light plus noise, $\# \mathrm{p}<0.01$ between groups same trial type, $\$ \mathrm{p}<0.05$ comparison to zero, $+\mathrm{p}<0.05$ compared to block $1,++\mathrm{p}<0.01$ compared to block 1 ) 


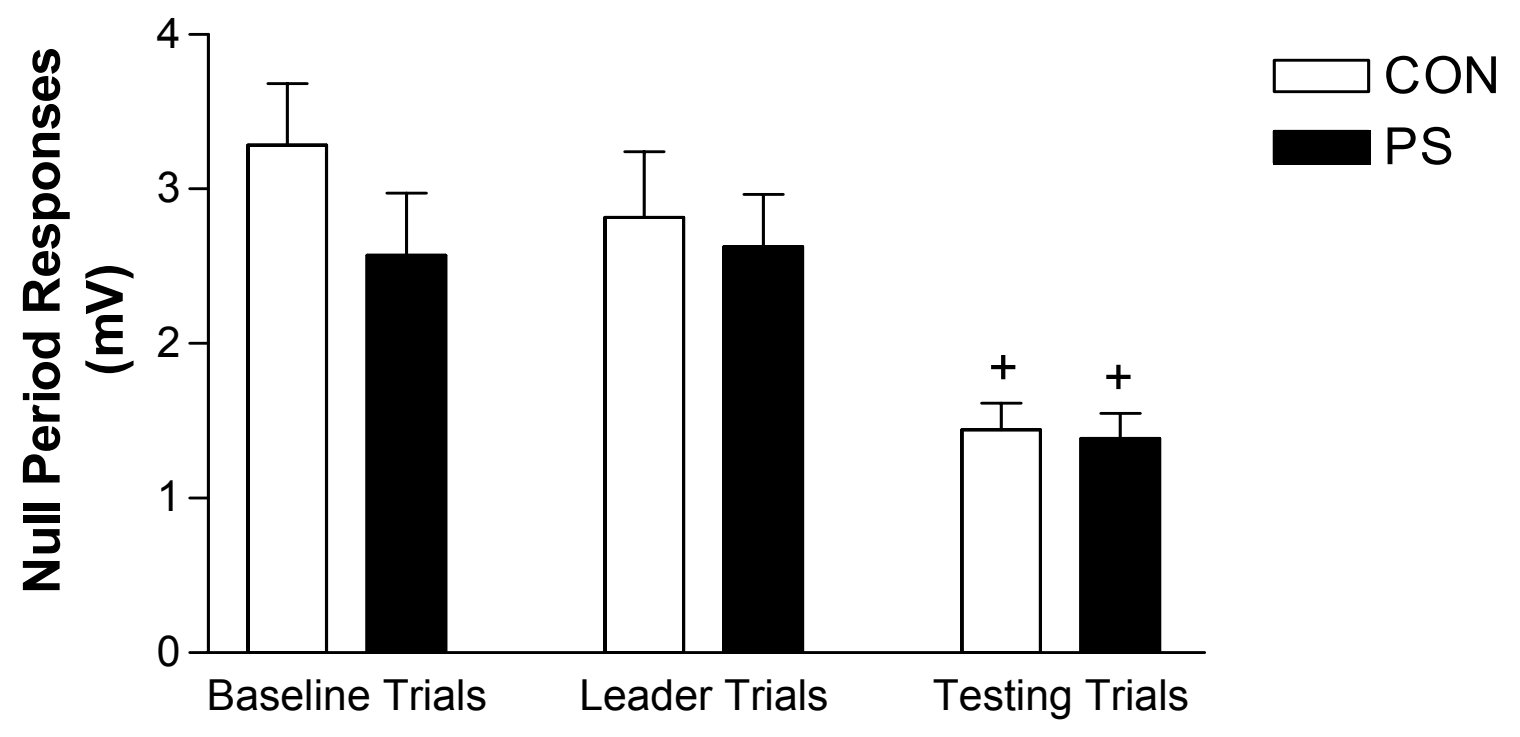

Figure 19. Null period responding from the experiment shown in Figure 18. There was a depression in null period responding during the leader trials in both groups, but no differences were detected between the groups. The values are means \pm S.E.M. (see results for statistical details; $+p<0.01$ within group compared to baseline and leader trials). 
Table 5.

\begin{tabular}{|c|c|c|c|}
\hline Group (Experiment) & Baseline trials & Leader trials & Testing trials \\
\hline CON (A) & $34 \pm 1$ & $18 \pm 0.5^{*}$ & $19 \pm 1 *$ \\
\hline PS (A) & $30 \pm 1 \wedge$ & $17 \pm 0.5^{*}$ & $15 \pm 0.3 * \wedge$ \\
\hline CON (B) & $36 \pm 2$ & $28 \pm 3 *$ & $16 \pm 1 * \#$ \\
\hline PS (B) & $33 \pm 2$ & $22 \pm 2 * \&$ & $14 \pm 1 * \#$ \\
\hline
\end{tabular}

Table 5 shows the average latency to peak startle responses, in milliseconds, from two FPS experiments conducted using two days of training at $0.5 \mathrm{~mA}$ shock intensity. Experiment A was also shown in Figure 15, Part A and Experiment B was shown in Figure 19. Latencies were faster during the leader and testing trials of both experiments when compared to baseline values. Latencies during the leader trials of Experiment B were shorter than the latencies during the testing trials of Experiment B. The difference between the two experiments was the contextual changes made during Experiment B. The reported values are means \pm S.E.M. (See results for details; ${ }^{*} p<0.01$ within group compared to baseline, $\wedge \mathrm{p}<0.01$ between groups same trial type, $\# \mathrm{p}<0.01$ within group compared to leader trials, $\& \mathrm{p}<0.05$ between groups same trial type). 


\section{Part E, Signal Transduction}

\section{$\underline{5-\mathrm{HT}_{1 \mathrm{~A}}} \underline{\text { Receptor Binding Assays }}$}

Figure 20 shows the staturable binding of ${ }^{3} \mathrm{H}-8$-OH-DPAT in block dissected tissue from CON and PS rats. The amount of staturable binding $\left(\mathrm{B}_{\max }\right)$ and the affinity $\left(\mathrm{K}_{\mathrm{D}}\right)$ were determined by non-linear regression of the data using GraphPad Prism ${ }^{\circledR}$. Prism ${ }^{\circledR}$ automatically compares the parameters of interest using Student's t-test. In the amygdala samples, the calculated $\mathrm{B}_{\max }$ and $\mathrm{K}_{\mathrm{D}}$ values for the CON and PS rats were $48.1 \pm 15.2$ $\mathrm{fmol} / \mathrm{mg}$ protein and $6.9 \pm 4.1 \mathrm{nM}$ and $50.2 \pm 5.2 \mathrm{fmol} / \mathrm{mg}$ protein and $4.5 \pm 1.0 \mathrm{nM}$, respectively. Differences between the groups were not detected in this analysis $\left(\mathrm{B}_{\max }: \mathrm{t}=\right.$ $\left.0.1297, \mathrm{p}=0.8974 ; \mathrm{K}_{\mathrm{D}}: \mathrm{t}=0.5692, \mathrm{p}=0.5720\right)$.

In the hippocampus, the PS rats had a significant decrease in $\mathrm{B}_{\max }$ when compared to the $\mathrm{CON}$ rats ( $\mathrm{t}$-test; $\mathrm{t}=2.075, \mathrm{p}=0.0422)$. The calculated $\mathrm{B}_{\max }$ was $105.2 \pm 10.5$ $\mathrm{fmol} / \mathrm{mg}$ protein for the PS rats and $139.0 \pm 12.5 \mathrm{fmol} / \mathrm{mg}$ protein for the CON rats. The calculated $\mathrm{K}_{\mathrm{D}}$ values of $4.5 \pm 1.4 \mathrm{nM}$ and $5.8 \pm 1.5 \mathrm{nM}$ for the PS and CON rats, respectively, were not different between the groups ( $\mathrm{t}$-test; $\mathrm{t}=0.6388, \mathrm{p}=0.5253$ ). 


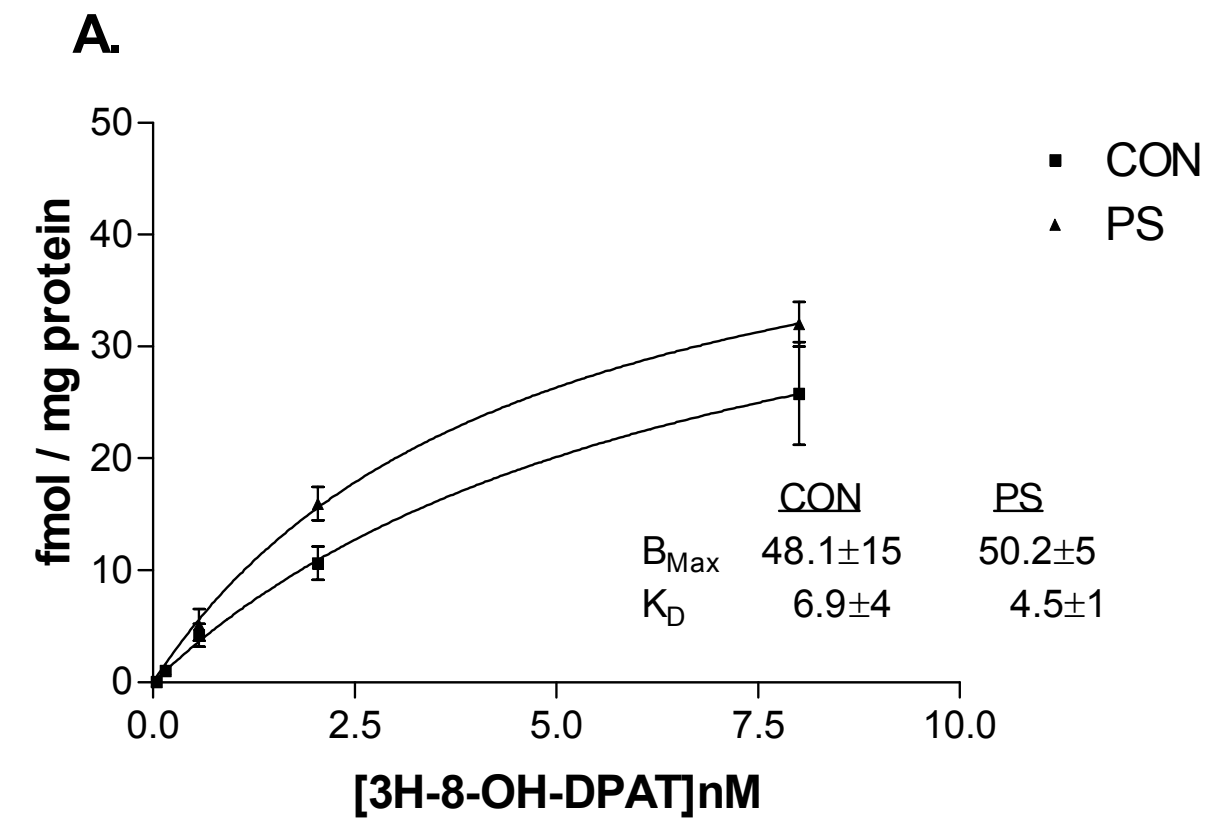

B.

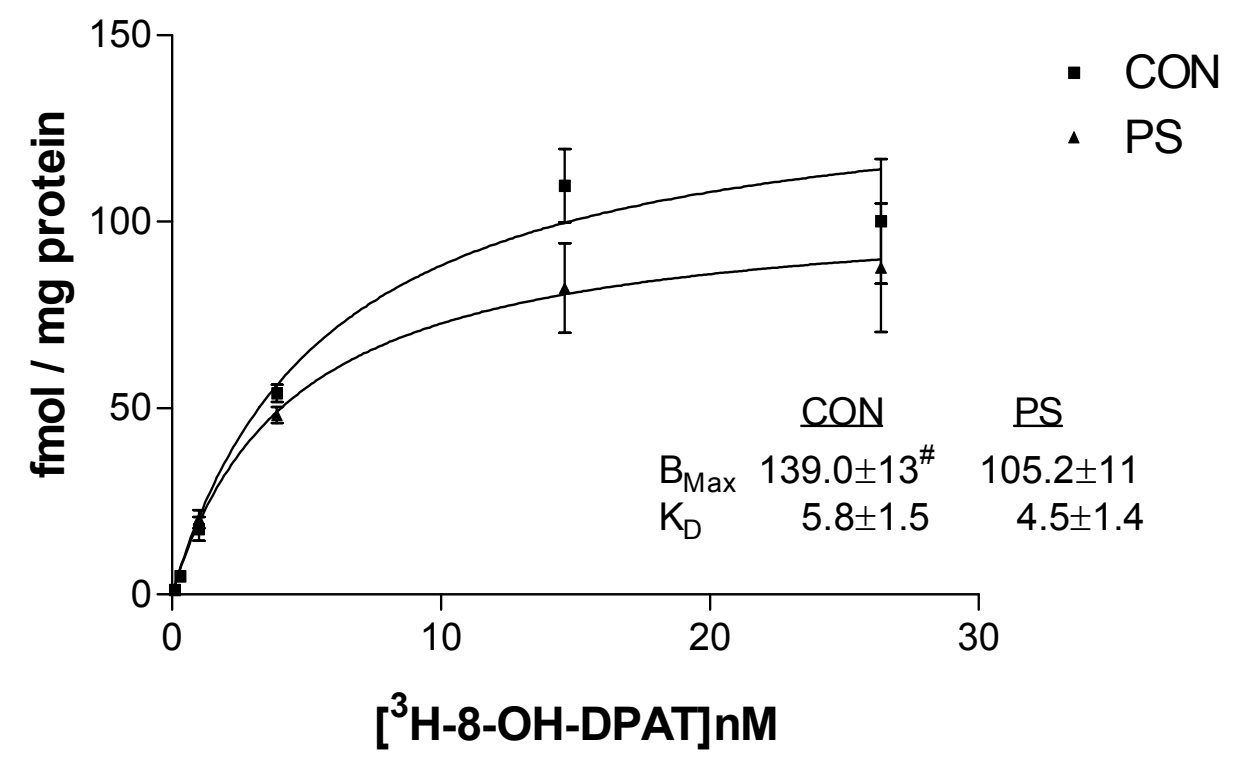

Figure 20. ${ }^{3} \mathrm{H}-8-\mathrm{OH}-\mathrm{DPAT}$ binding in block dissections of the amygdala and the hippocampus. A) Saturable binding in the amygdala. No differences were detected between the groups. B) Saturable binding in the hippocampus. PS rats were found to have a smaller $B_{\max }$ but the $\mathrm{K}_{\mathrm{D}}$ was unchanged compared to $\mathrm{CON}$ rats. There were 5-6 rats per group representing 2-3 independent litters. See results for details. ( $\# p<0.05$ between groups) 


\section{$\underline{\text { cAMP Production }}$}

Figure 21 shows basal and forskolin stimulated cAMP production in block dissections from CON and PS rats containing the amygdala and hippocampus. Each data set was analyzed by a Student's t-test since each pair of columns in the graph represents an independent experiment. No differences were detected between the groups in either brain region under basal conditions of cAMP production ( $\mathrm{t}$-test; $\mathrm{t}=0.6169, \mathrm{p}=0.5511$ for the amygdala and $\mathrm{t}=0.2266, \mathrm{p}=0.8253$ for the hippocampus). There was the expected increase in the amount of cAMP due to forskolin stimulation of adenylyl cyclase in both brain regions, but no differences were found between PS and CON rats in either brain region ( $\mathrm{t}$-test; $\mathrm{t}=1.362, \mathrm{p}=0.2$ for the amygdala and $\mathrm{t}=1.313, \mathrm{p}=0.2217$ for the hippocampus). Interestingly, there appears to be more basal production of cAMP in the amygdala when compared to the hippocampus $(>3 \mathrm{x})$. However, as pointed out above, the experiments were conducted independently so statistical comparisons were not made of this data. 


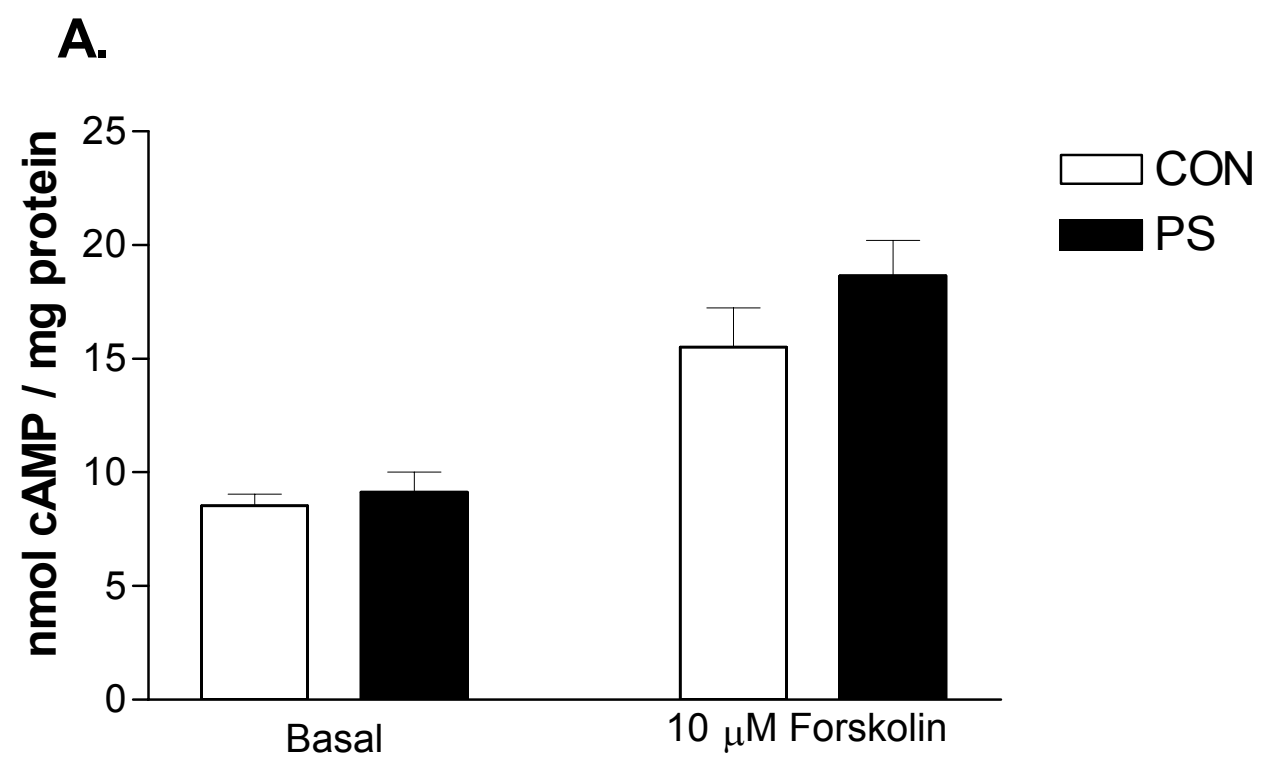

B.

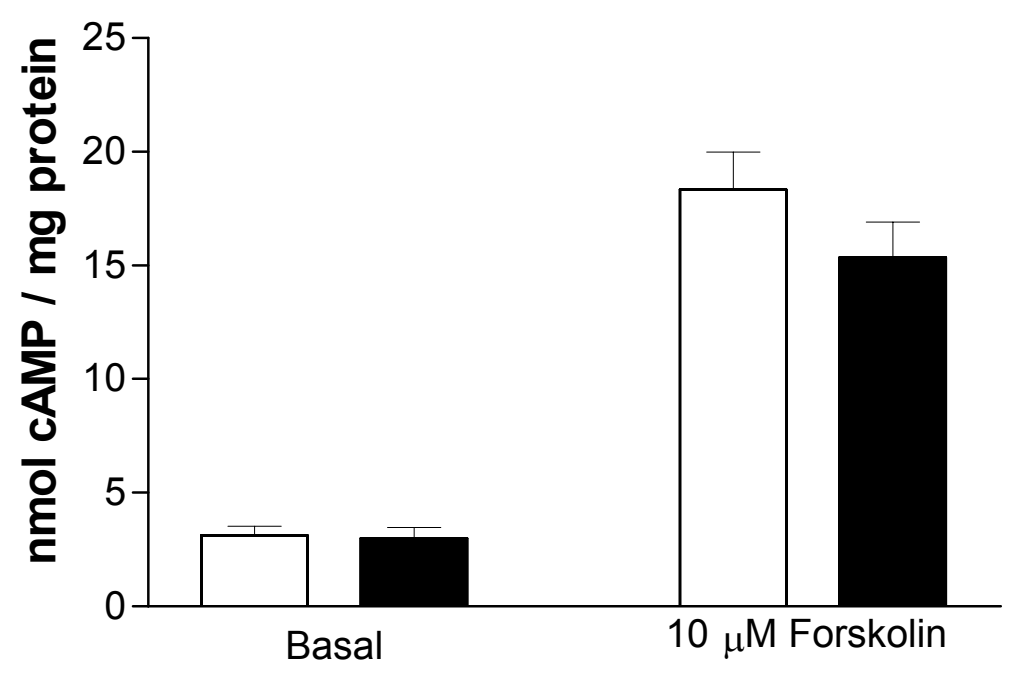

Figure 21. cAMP production in block dissected tissue samples from amygdala and hippcampus. A) Basal and Forskolin stimulated cAMP production in the amygdala. No differences were detected between the groups. B) Basal and Forskolin stimulated cAMP production in the hippocampus. No differences were detected between the groups. The were 6 rats per group, representing 3 independent litters per group. See results for more details. 


\section{Development}

\section{DISCUSSION}

Psychological trauma during human pregnancy has been associated with adverse outcomes for the infant, such as low birth weight and shorter gestational periods (Wadhwa et al., 1993) (Rothberg and Lits, 1991) (Newton and Hunt, 1984). Accordingly, we wanted to determine if our stressful intervention during pregnancy resulted in these types of outcomes. In our model of prenatal stress, length of gestation and average litter size were not different between the PS and CON litters (Table 1). Interestingly, over the period of our record review the $\mathrm{CON}$ group tended to have larger litter sizes, but this was not statistically significant (Table 2a). Finally, body weights and AG distance were found not to be different between naïve PS and CON rats over a range of ages from P1 to P65 (Figures 5 and 6).

A few reports utilizing rodent prenatal stress models have been published describing the effects of the chronic stress on length of gestation, birth weight and AG distance of the pups, which have been negative in this regard (Peters, 1986a) (Kinsley and Svare, 1987) (Poltyrev et al, 1996). On the other hand, there are also reports of low birth weight and shorter AG distances (Williams et al., 1998). Differences in these parameters are usually seen with more severe prenatal stressors (e.g. restraint three times daily) [for a review see (Weinstock et al., 1988)]. Additionally, maternal stress during gestation, such as restraint or social stressors, is known to result in resorption of fetuses in rodent species which would be detected as a decrease in litter size by our recording methods (Wise and Eldred, 1986) (Sugino et al., 1994). The resorption process might explain the slight tendency for the CON group to have larger litter sizes as compared to the PS group. 
However, given the mild nature of our gestational stress paradigm as compared compared to more involved stressing protocols, it is not surprising that significant changes in basic parameters of gestation are not seen.

As might be expected, body weight at the P1 age decreased as a function of litter size (Table2b). This may point to a confounding factor for our studies that relates to prenatal nutrition and subsequent development of the fetuses and young offspring. $A$ priori, larger litter sizes in utero get proportionally less nutrition per fetus than smaller litter sizes in utero. This is not meant to imply that the nutritional intake of the mother is inadequate since viable pups are born and survive to adulthood. However, the data in Table $2 \mathrm{~b}$ for clearly indicate that smaller litter sizes result in larger pups at birth suggesting that the growth process is accelerated.

It has been shown that smaller litter sizes in utero (by isolation of one uterine horn) correlate with larger body sizes, heavier brain weights, accelerated acheivement of developmental milestones (e.g. pinnae opening) and better performance on behavioral measures in the pups during the first week of life (Wehmer F and Jen KL, 1978). Thus, if the CON group tends to have larger litter sizes (Table 2a), then this effect may be confounding experimental analyses. Finally, nutritional deficits in animal studies have been hypothesized to be linked to neurobiological problems related to psychiatric disorders such as schizophrenia (Butler et al., 1994). A reasonable methodological change in our model would be to impose a cut-off value, above which litters of either group would not be used. Further work is needed to accurately determine a cut-off value that lessens the presumed impact of large litter sizes yet allows reasonable production of subjects for research projects. 
Previous studies in our laboratory have shown that $\mathrm{CRH}$ content is increased in the amygdala and the hypothalamus of older, adult PS rats (Cratty et al, 1995) (White and Birkle, unpublished). We were interested in describing the ontogeny of this phenomenon by determining $\mathrm{CRH}$ content in preweanling and young adult rats. Using a micropunch dissection technique, we did not detect any differences in CRH content of the BNST, $\mathrm{CeA} / \mathrm{BLA}$ and $\mathrm{PVNH}$ in preweanling PS and CON rats (Figure 7). Using the same technique, we measured $\mathrm{CRH}$ content of these same brain regions, with the addition of the DRN, in PS and CON rats aged P25 to P65 (Figure 8). Again, no differences were detected in $\mathrm{CRH}$ content at these ages between PS and CON rats.

There are two related possibilities that may explain the current findings with respect to the previous findings. In the first study by Cratty et. al., the investigators utilized block dissected tissue to make their observations (Cratty et al, 1995). This raises the possibility that other brain regions, not investigated in the current study, are contributing to the difference in $\mathrm{CRH}$ content between PS and CON rats. The candidate brain regions for this effect, each containing copious amounts of $\mathrm{CRH}$, are the hippocampus, endopiriform cortex and median eminence (Palkovits et al, 1985) (Skofitsch and Jacobowitz, 1985). Nevertheless, this still does not provide a complete explanation. The unpublished study by White and Birkle did employ the micropunch dissection technique and managed to find small differences in total $\mathrm{CRH}$ content in the CeA/BLA and PVN in adult offspring. The difference between the present study and the previous study may be in the experimental history of the animals used in the studies. The rats used in the current study were all experimentally naïve, where CRH content was not different between PS and CON rats. Both of the previous studies utilized tissue taken from rats used in behavioral experiments. 
Thus, the differences in CRH content between PS and CON rats detected previously may be related to prior use in experiments and the stress involved in those procedures.

A mechanism for increases in CRH content in stressed PS rats may be HPA axis dysregulation. PS rats have greater plasma corticosterone responses to a variety of stressors than do CON rats. This finding is true for brief tailshocks and saline injections (Takahashi and Kalin, 1991) (Peters, 1982). Fride et. al. demonstrated that PS rats continued to show increased plasma corticosterone, when compared to CON rats, even after 8 days of repetitive exposure to an open-field (Fride et al, 1986). It is counterintuitive to expect glucocorticoids to increase $\mathrm{CRH}$ when they are known to participate in the negative feedback loop of the HPA axis. However, there is one report demonstrating that following footshock or cold water swim, CRH mRNA is increased (Lightman and Harbuz, 1993). Protein kinase A stimulation, via forskolin-induced elevations of cAMP, in primary amygdalar cultures also increases CRH production (Kasckow et al., 1997). Finally, psychologically stressing a rat by putting it in the presence of other rats being footshocked leads to increases in CRH mRNA in the amygdala but not the hypothalamus (Makino et al., 1999). These observations indicate that, despite the negative feedback loop, $\mathrm{CRH}$ production can be increased under the right circumstances in vivo.

In summary, the mild gestational stress employed during the present studies does not alter the length of gestation and litter size. Body weight and AG distance, over a range of postnatal ages, are also not affected by the gestational stress. Body weight at birth was found to be inversely proportional to litter size and may reflect important issues related to prenatal nutrition. Finally, CRH content in several brain regions is not affected by the 
gestational stress at the ages assessed in this study when the rats are naïve to experimental use.

\section{Preliminary Study of Chronic Mild Stress}

The previous observations regarding experimental use and CRH content, coupled with known HPA axis dysregulation in PS rats, led to the hypothesis that PS rats may be more susceptible to chronic mild stress than the $\mathrm{CON}$ rats. For a preliminary examination of this idea, we assessed our PS and CON rats while the rats were being used in other experiments (Table 3). In this situation, the other experiments became the stressors. In this

study, the rats were subjected to a variety of experimental manipulations from a young age, ranging from open field testing to footshock based fear-conditioning which occurred more or less on regular intervals. The primary outcome measure of interest was anhedonia, as measured by sucrose intake.

During the course of exposure to the other experiments, sucrose intake was found to be depressed in the PS group relative to the CON group during the daytime and nighttime assays over the 4 weeks that the measurements were conducted (Figure 9). This behavioral assessment used a two-bottle paradigm with tap water serving as the alternate choice. Water intake was found not to be different between the groups or as a function of time of day. Unfortunately, baseline measurements were not conducted in these sucrose intake determinations. Thus, we cannot judge the extent to which the chronic stress affected the sucrose intake in either group, i.e. there could have been preexisting differences in sucrose intake and sucrose intake remained unchanged despite the stressors. There is at least one report suggesting that saccharin preference is decreased by prenatal stress, so the 
suggestion of pre-existing differences cannot be ruled out entirely in this experiment (Keshet and Weinstock, 1995). Additionally, there was not an age-matched set of nonstressed rats to allow an assessment of sucrose intake as a simple function of age between the PS and CON groups.

However, another group of younger, naïve CON and PS rats was assessed for basal sucrose intake and differences were not found between the groups; although, sucrose and water intake values were generally lower than those reported in Figure 9 (See results). Although more work must be done, together these observations are consistent with the idea that sucrose intake in PS rats may be depressed to a greater extent by chronic stress than in CON rats.

Body weights were generally lower in the PS group over the course of this study, although specific differences were not detected by the post-hoc analysis. This finding, albeit weak, may be related to the chronic mild stress of experimental use since body weights were not different 4 weeks prior to the start of the sucrose assays (approximately the middle of the behavioral series, see Table 3). As noted previously, naïve PS and CON rats are not different in terms of body weight over a range of ages (Figures 5 and 6). This mild weight loss can result from the chronic stress. Willner has reported weight losses on the order of $10 \%$ compared to pre-stress baseline values in his studies (Willner et al., 1996).

It needs to be pointed out that Willner's paradigm is somewhat controversial. with at least three reports demonstrating negative findings. Part of the controversial nature of the paradigm stems from the reproducibility of the decrease in sucrose intake in response to chronic mild stress, something that even Willner has acknowledged in his own work 
following a change in laboratory locations (D'Aquila et al., 1997). Moreover, other investigators have reported negative results (Harris et al., 1997) (Matthews et al., 1995) (Forbes et al., 1996). The controversy also extends to the philosophical underpinnings of the model; for example, does decreased sucrose consumption actually indicate anhedonia if sucrose preference does not change? In fact, in addition to a review by Willner, a couple of informative commentaries debating the usefulness of this model of anhedonia appear in volume 134 of the journal Psychopharmacology (Willner, 1997) (Weiss, 1997) (Broekkamp, 1997). Despite the controversy, Willner and his colleagues have reported a number of different experiments using a variety of pharmacological assessments, two different rodent species and other physiological parameters not related to sucrose intake (e.g. intracranial self-stimulation) to argue their point that their chronic mild stress paradigm results in anhedonic rats (Cheeta et al., 1997) (Monleon et al, 1995) (Papp et al, 1996).

At sacrifice, adrenal glands from the chronically stressed rats were removed and the weights compared to adrenal glands taken from an age-matched set of naïve CON and PS rats (Table 4). No differences were detected across the four groups of rats or when the analysis was performed as merely a function of stress versus non-stress groups. There was a trend towards increased adrenal weights in the chronically stressed rats, but it did not reach statistical significance. Adrenal glands in rodents are known to hypertrophy during times of chronic stress (Blanchard et al., 1998) (Zelena et al., 1999). The lack of a difference between the stressed and non-stressed rats suggests the chronic mild stress used in the present studies was not robust enough to produce this effect, despite the presumed effect on sucrose intake. 
In our model of prenatal stress, young PS rats ( $\sim 60$ days) have been shown to have larger adrenal glands than CON rats (Ward et al, 2000). The lack of a detectable difference in adrenal weights between the naïve PS and CON rats in the present study implies that, as the rats age, the difference in adrenal gland size between the groups becomes less pronounced. The reason for this finding is unclear. However, an intriguing hypothesis is that, in general, rats are more susceptible to adrenal gland hypertrophy at younger ages in response to stressful events such as weaning and exposure to routine handling by animal care technicians. A key corollary to this hypothesis is that the PS rats are even more sensitive to this type of physiological plasticity than the CON rats, as has been previous reported by our laboratory. Some evidence supporting this hypothesis and its corollary comes from a report by Vallee et. al., where they demonstrated that age related changes in glucocorticoid secretion differed between PS and CON rats (Vallee et al., 1999). In this study, PS rats had greater corticosterone levels following restraint stress than the CON rats, but only at the younger ages (4 months). Additionally, basal corticosterone levels, although not different between the groups at the youngest and oldest ages, were elevated more than 3 times at the oldest age ( 24 months) compared to the youngest age (Vallee et al, 1999). Thus, it appears that adrenocortical activity increases with age regardless of prenatal condition. These observations may provide a rationale for the lack of difference in adrenal gland weights between the CON and PS rats in the present study.

In conclusion, the PS rats seem to be more susceptible to chronic mild stress in terms of depressed sucrose intake. However, more studies are needed to definitively prove this point, since baseline sucrose intake values for the stressed and non-stressed groups of rats were not obtained. The effect of prenatal stress on adrenal gland weights was not 
found in this study, as previously reported in younger animals. Some available evidence implies that the physiology of adrenal gland hypertrophy is complex and that it relates to age, stress and prenatal condition. Certainly, more studies are needed to determine the validity of this hypothesis.

\section{Acoustic Startle Responding and Serotonergic Neurotransmission}

Our laboratory has demonstrated that PS and CON rats do not differ with regard to normal acoustic startle responding (White and Birkle, 2001). However, these studies were conducted using only one decibel level, 95 decibels. Exposure to loud sounds is known to be stressful and can change such things as eating behaviors (Krebs et al., 1996) (Krebs et al., 1997). Accordingly, acoustic startle responding was assessed over a range of decibel levels to determine if PS rats would respond differently at higher decibel levels than CON rats (Figure 10). Differences were not detected between PS and CON rats with regard to peak startle responses or latency to peak startle response at any of the decibel levels tested.

The data in Figure 10a show that startle responding increases linearly from 95 decibels up to about 110 decibels where responding does not appear to increase in a linear fashion. The lack linearity at the higher decibel levels is probably due to habituation of the startle response as the session progresses since habituation is known to be more pronounced with stimuli at higher decibel levels (Pilz and Schnitzler, 1996). Habituation is a decremental process that degrades the acoustic startle response with repetitive presentation of the stimulus(Pilz and Schnitzler, 1996). The inset shown in Figure 10a shows a decline in startle responding to the same stimuli as the session progressed, in keeping with this idea (also see results). Thus, in addition to peak startle responding not 
being different over a range of decibel levels between the groups, habituation of the peak startle response over a range of randomly presented decibel levels does not appear to be different between the groups.

One might argue that a weakness with this experiment is that a variety of decibel levels were presented to the rats within the same test session. The range of decibel levels presented to the rats encompasses decibel combinations that can be used in pre-pulse inhibition experiments. Pre-pulse inhibition is a paradigm in which a weak pre-stimulus is presented just prior to a stronger stimulus and, as a result, the response to the stronger stimulus is inhibited (Koch, 2000). However, for the pre-pulse to be effective at reducing the magnitude of the response to the next stimulus, it must be presented 30 to 500 milliseconds prior to the stronger stimulus (Koch, 2000). Since the present experiment used an inter-trial interval of 30 seconds, there are unlikely to be effects resembling prepulse inhibition on the peak startle response.

Figure 10b shows the results of randomly presented acoustic stimuli on the latency to peak startle. As noted before, the statistical analysis did not detect any effect of decibel level or group; in fact, the latencies are quite similar across each decibel level. Therefore, one can conclude that within the confines of this experiment, latency to peak response is not dependent upon the decibel level of the stimulus as is the peak response. This is an intriguing finding, since the usual expectation is to see a decrease in latency times as peak startle responses increase (Pilz and Schnitzler, 1996). Furthermore, evidence from prepulse inhibition studies suggests that latency to peak response and the peak response are controlled by separate mechanisms (Koch, 2000). If this proposition is true, then perhaps 
this variable decibel experiment also provides evidence of the dissociation between these two parameters of the ASR.

Previous studies from our laboratory have shown that with a high dose of yohimbine (e.g. $5 \mathrm{mg} / \mathrm{kg}$ ), acoustic startle responding is not elevated in the PS rats to the same degree as with CON rats (White and Birkle, 2001). Tests with RS79948-197 HCl, a selective alpha $a_{2}$-adrenoceptor antagonist, during that same study determined that this effect was probably not due to differences in the PS rat's sensitivity to yohimbine's antagonism of $\alpha_{2}$ adrenoceptors. Unfortunately, yohimbine is capable of interacting with several neurotransmitter systems, including the adrenergic, serotonergic and dopaminergic systems (Smith et al., 1996) (Scatton et al., 1980) (Lattimer et al., 1984). In particular, yohimbine has been shown to act as a partial agonist at 5- $\mathrm{HT}_{1 \mathrm{~A}}$ receptors (Arthur et al, 1993). The fact that yohimbine can interact with $5-\mathrm{HT}_{1 \mathrm{~A}}$ receptors is also supported by drug discrimination testing revealing that 8-OH-DPAT can generalize to yohimbine (Winter and Rabin, 1992).

The present studies sought to determine if the difference in response to yohimbine between the PS and CON rats was due to differences in $5-\mathrm{HT}_{1 \mathrm{~A}}$ receptor mediated events. For these studies, two well characterized ligands were available. The antagonist, WAY100635 , is highly selective for the $5-\mathrm{HT}_{1 \mathrm{~A}}$ receptor and has extremely low efficacy (Fletcher et al, 1996) (Gozlan et al, 1995). The agonist, 8-OH-DPAT, has been regarded for many years as being very selective for $5-\mathrm{HT}_{1 \mathrm{~A}}$ receptors with high efficacy; however, it does have affinity for $5-\mathrm{HT}_{7}$ receptors at low micromolar concentrations (Fletcher et al, 1996) (Wood et al., 2000).

Figure 11a shows the peak startle responses for PS and CON rats treated with various doses of 8-OH-DPAT. Treatment with 8-OH-DPAT produced an increase in startle 
responding at the highest dose in the PS and CON rats compared to their respective vehicle responses. Additionally, the post-hoc analysis also revealed that the responses of the PS rats at the $8 \mathrm{mg} / \mathrm{kg}$ dose was greater than that of the CON group. A follow-up study using a $16 \mathrm{mg} / \mathrm{kg}$ dose of 8 -OH-DPAT yielded even higher peak responses responses in both groups and the same difference between the groups (data not shown; Skinner, Griffin and Birkle, unpublished observations). Additionally, in the present study, latency to reach peak startle amplitude was increased in a dose-dependent fashion by 8-OH-DPAT in the PS rats (Figure 12a). Curiously, the $\mathrm{CON}$ rats did not show a consistent change in terms of the effects of 8-OH-DPAT on the latency parameter. Svensson reported increases in peak startle responding and latency to peak startle response with systemic treatment of 8-OHDPAT with a similar range of doses (Svensson, 1985). These observations demonstrate that the PS rats are more sensitive to the effects of 8-OH-DPAT on the acoustic startle response than the CON rats. The fact that the PS rats are more sensitive to a serotonergic agonist is consistent with reports by D.A. Peters from the mid-1980's showing enhanced behavioral responses to 5-hydroxy-L-tryptophan (Peters, 1986a).

It is interesting that the PS rats are more sensitive to the effects of 8-OH-DPAT in ASR testing and it indicates that there is a leftward shift in the dose-response curve for the peak response and the latency parameter. Obtaining the plateau of the dose-response curve in order to prove a leftward shift would be the logical next experiment. Unfortunately, this is not possible with 8-OH-DPAT due to the prevelance and severity of the serotonin syndrome as higher doses are used. This syndrome manifests itself as flattened posture, increased forward movement, wet-dog shakes and temperature dysregulation (Blanchard et al., 1993). In fact, during the course of the follow-up study alluded to previously, several 
$\mathrm{CON}$ rats died, probably from temperature dysregulation induced by the 8-OH-DPAT. The temperature dysregulation manifests itself as hypothermia, which can be blocked by WAY100635 indicating the involvement of 5- $\mathrm{HT}_{1 \mathrm{~A}}$ receptors (Trillat et al., 1998). Interestingly, chronic corticosterone treatment attenuates the hypothermic response to 8-OH-DPAT, and given the noted HPA-axis hypersecretion of coritcosterone in PS rats, this may explain why no PS rats died in the course of the follow-up study (Takao et al., 1997). In spite of that, the motor symptoms of the serotonin syndrome may interfere with the generation of the acoustic startle response and the hypothermia can obviously endanger the life of the rat, making the generation of a full dose-response curve very difficult.

The mechanism for this increased sensitivity on the part of the PS rats is unclear, but there are at least two possible explanations. The first consideration is a difference in metabolism of 8-OH-DPAT between PS and CON rats. In the rat, 8-OH-DPAT is excreted largely as a glucuronidated molecule with a terminal half-life of $\sim 1.5$ hours (Mason et al., 1995). Additionally, stress is known to alter liver function enzymes such that drug levels can be changed (Stitzel and Furner, 1967) (Stitzel, 1972). Although no reports exist, if such actions can occur via prenatal stress or by the interaction of prenatal stress with other stressful events in the life of a rat, then PS rats would obviously metabolize drugs differently. In the case of PS rats, the altered metabolism may result in higher plasma levels of 8-OH-DPAT and, consequently, larger responses at a given dose. This explanation, though plausible, is not completely satisifactory since elevations in ASR are apparent within minutes of the i.p. injection, before the effects of altered metabolism would be expected to be seen (time course data not shown). The second consideration, which will 
discussed in more detail below, are alterations in discrete elements of the serotonergic system such as changes in receptor density in key brain nuclei associated with the ASR.

On the other hand, WAY-100635 does not affect acoustic startle at all in either group (Figure 11b). No dose related changes were detected in this study in terms of peak startle amplitude, in keeping with control data from an FPS study published by Joordens et. al. (Joordens et al., 1998). Evaluation of the latency to peak response gave similar findings (Figure 12b). Latency times seemed to be depressed in the CON group under vehicle treatment as compared to what was seen in the 8-OH-DPAT study, but this was not statistically different (See results). It should be noted that a weakness in this experiment with WAY-100635 was the high variability of responding which was probably related to the small number of rats tested.

The current results with 8-OH-DPAT, particularly with the PS rats, create a curious dilemma with data interpretation. The magnitude of the acoustic startle response increases in states of fear or with the administration of anxiogenic drugs (Davis, 1984). Therefore, the effects of 8-OH-DPAT on peak startle responding in either group can be interpreted as an anxiogenic response. Conversely, the present study has demonstrated that decreases in latency to peak response occur in states of fear and, therefore, must be interpreted as an anxiogenic response (see Table 5 and the discussion below under Fear Conditioning). The PS rats demonstrated a clear increase in the latency parameter with increasing doses of 8OH-DPAT. There is not any published evidence to argue that the increase in latency to peak startle response is actually an anxiolytic response, but the increase in latency is not consistent with a presumed anxiogenic effect of 8-OH-DPAT on peak startle responding given the findings in the fear-conditioning studies. Furthermore, as noted above, peak 
response and latency to peak response are generally related by a negative correlation (Pilz and Schnitzler, 1996). In fact, as the startle responding habituates (i.e. decreases with repetitive presentation) there will be a general increase in latency to peak response (Pilz and Schnitzler, 1996). Thus, the interpretation of the data must include some explanation for the apparent disconnect between peak response and latency to peak response.

It is known that ablation of the DRN results in an increase in peak acoustic startle responses (Davis and Sheard, 1974). The DRN supports a rich population of 5-HT $1 \mathrm{~A}$ autoreceptors, that, when activated, decrease neuronal activity (Forster et al, 1995). It could be hypothesized that activation of these receptors decreases inhibitory serotonergic tone supplied to the primary acoustic startle pathway which, in turn, results in an elevated startle response. Alternatively, there could be a direct effect of 8-OH-DPAT on the spinal cord that results in elevated startle responses. $5-\mathrm{HT}_{1 \mathrm{~A}}$ receptors are found in the spinal cord and 5-HT applied directly to the spinal cord is known to elevate responses to acoustic stimuli, although it is unknown which serotonergic receptor mediates this action of 5-HT (Astrachan and Davis, 1981). Furthermore, in an unrelated paradigm, a control experiment showed that 8-OH-DPAT applied directly to the spinal cord increased reflexive twitches in the gastrocnemius muscle caused by electrical stimulation of the sural nerve in spinalized rabbits (Ogilvie and Clarke, 1998). Thus, there are at least two locations in the central nervous system for a direct effect of 8-OH-DPAT can enhance peak startle responding and two locations were prenatal stress may have changed neural function to yield greater responses in the PS rats. Changes caused by prenatal stress might include alterations in receptor density or alterations in second messanger signalling. 
There is also the consideration of 8-OH-DPAT's low micromolar affinity for $5-\mathrm{HT}_{7}$ receptors, which has been elucidated by in vitro binding assays using cells transfected with 5-HT 7 receptors (Wood et al, 2000). Therefore, at high systemic doses of 8-OH-DPAT, the possibility of cross-over to the $5-\mathrm{HT}_{7}$ system cannot be excluded. Unfortunately, there are no known selective drugs for this receptor and, consequently, there are no published investigations of the involvement of the $5-\mathrm{HT}_{7}$ system with the acoustic startle response. However, the distribution of $5-\mathrm{HT}_{7}$ receptors, as determined by northern blotting and in situ hybridization, indicates that the receptors are not localized to any great extent in nuclei associated with the acoustic startle response. The primary sites of expression in the brain are the hypothalamus and thalamus (Lovenberg et al., 1993). In this same study by Lovenbrrg et. al., weak expression of 5- $\mathrm{HT}_{7}$ receptors was detected in "medulla" but they did not elaborate on what structures might contained in this tissue preparation (Lovenberg et al, 1993). For example, did it include more caudal areas of the pons, perhaps parts of the RPC? The investigators also did not examine samples of spinal cord in this experiment. Thus, an involvement of 5- $\mathrm{HT}_{7}$ receptors in acoustic startle responding cannot be ruled out, but given the limited distribution uncovered so far, it seems unlikely that these receptors would contribute to any great extent to 8-OH-DPAT mediated increases in peak startle responses.

The effect of 8-OH-DPAT on the latency to peak startle response is more difficult to explain due to the paucity of literature on this parameter and the factors that control it. The latency parameter reflects the speed of transmission of the auditory stimulus from the Organ of Corti in the ear to the skeletal muscles that produce the response (Koch, 2000). Interestingly, the PS rats, under vehicle conditions, have faster latencies than the CON rats 
when auditory stimuli of a single decibel level are presented (95dB SPL), which is consistent with the baseline startle data shown in Table 5 but not with the WAY 100635 study (Figure 12a). The reason for this observation is not clear but the vehicle response data imply that prenatal stress improves synaptic efficacy to speed up neurotransmission through the startle pathway. Furthermore, the data imply that 5- $\mathrm{HT}_{1 \mathrm{~A}}$ receptors are involved, either directly or indirectly, with this enhanced synaptic efficacy in the PS rats since activation of these receptors retards the flow of information through the pathway.

In conclusion, the current results support the idea that acoustic startle responding and habituation of the response, is generally unchanged in PS rats, even at higher decibel levels. Another finding was the faster latency times of the PS rats. This is an admittedly weak finding since it is not always detected. However, the drug-modulated startle data presented here are not consistent with PS rats being less sensitive to yohimbine's agonist activity at $5-\mathrm{HT}_{1 \mathrm{~A}}$ receptors, since $8-\mathrm{OH}-\mathrm{DPAT}$ is increasing startle to a greater extent in the PS group (yohimbine had the opposite effect). The results are also not consistent with yohimbine depressing startle in the PS group via $5-\mathrm{HT}_{1 \mathrm{~A}}$ blockade, since WAY-100635 does not have an effect on startle in either group.

The response difference between PS and CON rats to a $5 \mathrm{mg} / \mathrm{kg}$ dose of yohimbine may be explained by increased sensitivity of the PS rats to $\alpha_{1}$ adrenoceptor modulation. Yohimbine has affinity for these receptors so an interaction with these receptors with systemic dosing cannot be excluded (Lattimer et al, 1984). Furthermore, activation of $\alpha_{1}$ adrenoceptors in the spinal cord by intrathecal phenylephrine increases the peak startle response (Astrachan et al., 1983). So, assuming that yohimbine is acting as an $\alpha_{1}$ adrenoceptor antagonist in the spinal cord, one might expect increases in peak startle 
responding caused by descending noradrenergic projections to be antagonized by high doses of yohimbine and yield the paradoxical inhibition in startle responding. This hypothesis also assumes that the contributions of the $\alpha_{2}$ adrenoceptors in the spinal cord, which do increase startle when yohimbine is directly applied, are outweighed by the influence of $\alpha_{1}$ adrenoceptors particularly in the PS rats (Davis and Astrachan, 1981). Certainly, given the number of interactions that yohimbine has with a variety of neurotransmitter systems, the complexity of the problem cannot be over-emphasized. The final answer will only come with further pharmacological analysis using more selective drugs directed at specific regions of the central nervous system.

\section{Conditioned Fear}

Fear-potentiated startle experiments were conducted to ascertain whether PS rats would demonstrate greater responses to a conditioned stimulus. The first series of experiments did not yield potentiation in either the PS or CON rats (Figure 13). Two variables were adjusted in these experiments: shock intensity and number of training trials. It is important to note that training context and testing context were exactly the same in these experiments. Neither of these adjustments resulted in potentiation, i.e. percent difference scores greater than zero (Figure 13). In fact, at the $0.5 \mathrm{~mA}$ shock intensity, the difference scores were actually negative indicating that many of the responses to the light + noise trials were less than the responses to the noise alone trials.

Nevertheless, two interesting observations were uncovered during these experiments. Figures 14 and 15 show peak startle responses and null period responses taken from the experiments using $0.5 \mathrm{~mA}$ of shock intensity. The first discovery was that the rats had increased peak startle responses regardless of trial type during the second half 
of the test after two training days (Figure 14). This is the portion of the test when the conditioned stimulus is presented. After four training days, this effect is quite pronounced in the PS group, as indicated by a large increase in startle responding during the testing trials compared to the $\mathrm{CON}$ rats (Figure14). These observations indicate that the light has acquired a fearful association since peak startle responding is selectively elevated during the portion of the test when it is presented to the rats. Furthermore, these observations suggest that the state of fear induced by the light during the testing trials is not easily extinguished from trial to trial since positive difference scores could not be calculated (Figure 13).

This phenomenon has been reported before with the FPS paradigm, but is not well understood (Joordens et al., 1997). Obviously, simply increasing the number of CS-US pairings during training does not resolve the issue and, in fact, only serves to enhance a generalized increase in peak startle responding in the PS rats (Figure 14). On the other hand, Davis and his colleagues have shown that when the FPS paradigm is working properly in their laboratory, the conditioned state of fear is greatest during the light cue, particularly when the shock would have been presented, and disappears very soon after the light cue is extinguished (Davis et al., 1989). Such a pattern of conditioned fear responding would allow the calculation of a positive difference score. As will be discussed below, the lack of a positive difference score in these two experiments may be related to subtle issues of context.

The findings in Figure 14 are consistent with the observation that, in general, startle responding can be increased following prior experience of footshock in the same context as testing, particularly if the light cue has become part of the context (Richardson and 
Elsayed, 1998). This effect after either two or four training days may be mediated by the CRHergic system, which is known to be active in states of fear and i.c.v. administered CRH is known to elevate ASR (Dunn and Berridge, 1990) (Kalin and Takahashi, 1990) (Liang et al, 1992).

With regards to the peak startle data shown for four training days, PS rats have documented increases in CRHergic system function, such as increased release in ex vivo amygdala preparations, and CRH content of the amygdala (Cratty et al, 1995) .

Furthermore, repeated footshock induced stress or several days of corticosterone treatment facilitates CRH-potentiated startle (Lee et al., 1994) (Pelton et al., 1997). There is also published evidence that the degree of fear-conditioning positively correlates with the amount of circulating corticosterone as well as the shock intensity used during training (Cordero et al., 1998). Thus, the current findings support the idea that the CRHergic system is more active in the PS rats and, coupled with deficits in HPA axis negative feedback, may be responsible for the dramatic increase in startle responding after four days of footshock training.

The null period responses from these experiments provided the other interesting observation from these experiments (Figure 15). The null period response serves as an index of voluntary motor activity during the test session (Plappert et al, 1993). In the PS group, after only 2 training days, null period responding was markedly depressed during the leader trials compared to the baseline value and this was true for testing trials as well. In contrast, the CON group's null period responding after 2 training days was only slightly depressed during the leader trials and seemed to be increasing during the testing trials. These effects were also evident after 4 training days (Figure 15). 
The depression in null period responding of the PS rats after two or four training days occurred initially during the leader trials of the test session. In contrast, in either experiment, the CON rats did not have marked depression in null period responding during the leader trials. A plausible hypothesis was that the PS rats were freezing more to context as a result of the footshock based training in that same context. Unfortunately, the short duration of the null recording period relative to the length of the test session as well as the restrictions on movement imposed by the testing equipment were weaknesses in supporting this hypothesis.

A traditional, observationally based conditioned freezing experiment was conducted to determine if PS rats were indeed capable of greater behavioral inhibition to context (i.e. the context is the conditioned stimulus) following an aversive experience 24 hours earlier. Figure 16 shows the data from that experiment. Rears were assessed as a measure of voluntary motor activity and were high during the pre-shock period. The data show that freezing was practically non-existent in both groups during the pre-shock period. Immediately following the shocks, freezing increased dramatically in both groups but to a greater extent in the PS group. As expected, rearing behavior was severely attenuated. The next day, freezing was still evident in both groups and still greater in the PS group. Rears were increasing the next day but only in the CON group. These observations are consistent with the data in Figure 15 and support the notion that PS rats are more sensitive to contextual cues after even a brief training session.

Somewhat in contrast to our results, Takahashi et. al. saw increased freezing in PS rats in the immediate aftermath of foot-shock presentation but did not see increased freezing when the PS rats were placed back into the context 24 hours later (Takahashi et al, 
1992a). The disagreement between our studies is probably due to methodological differences. The former study exposed the rats to the testing context for 2 days prior to shock presentation; whereas, in our study, the rats were shocked during the first visit to the chamber. Perhaps the lack of increased freezing 24 hours after footshock in the previous study was due to the phenomenon of latent inhibition where repeated exposure to the stimulus (e.g. the context) prior to training dampens the conditioning effect [for review (Lubow R.E., 1989)]. In fact, at least one report has been published showing that latent inhibition can contribute to the attenuation of conditioned freezing to context (Cruz et al., 1993).

Another freezing experiment was conducted that evaluated the rats' ability to freeze to an explicit cue, a light (Figure 17). After training, freezing was significantly increased in the presence of the light in both groups; however, PS rats and CON rats were immobile to the same extent. It is worth noting that the degree of freezing obtained to the light cue in this experiment is consistent with the published study from which the methods were derived (Phillips and LeDoux, 1992). Additionally, there was a high degree of freezing to the context in both groups, although less than to the light cue. There were no differences detected between the groups under either condition. The fact that there was a significant increase in freezing to the context after training, despite some changes in context, may be a result of the location of the conditioning chamber being the same from training to testing (See methods).

The lack of a difference between the groups in freezing to the light cue is probably a result of the long lived nature of the freezing response and the short duration of the observational periods (Blanchard and Blanchard, 1969) (Phillips and LeDoux, 1992). It is 
is worthwhile to point out that freezing in rodents does not necessarily have to occur as an extended period of total immobility, rather freezing can occur in discrete bouts that are periodically interrupted by head swiveling, sniffing or walking short distances. This statement is supported by the fact that, in the experiment shown in Figure 16, the rats were observed for 20 seconds but were not immobile for the whole period of observation. Nevertheless, future behavioral inhibition experiments using an explicit light cue will have at least two methodological changes: 1) change the location of the conditioning chamber between training and testing and 2) incorporate longer periods of observation after the light cue has been extinguished. These two changes would maximize the difference in freezing between the context and cue as well as expose differences between PS and CON rats that are consistent with those seen in the experiment shown in Figure 16.

An explanation for increased behavioral inhibition to contextual cues on the part of the PS rats may also be explained using the same rationale as described above for the increases in peak startle responding seen after 4 days of training. Freezing behavior can be blocked using $\mathrm{CRH}$ antagonists, indicating the involvement of the CRHergic system (Kalin and Takahashi, 1990). And, as noted previously, increases in plasma corticosterone are positively correlated with fear-conditioned behavior, in particular, defensive freezing (Cordero et al, 1998). Thus, given the known elevation of plasma corticosterone following stressors as well as the enhanced activity of the CRHergic system in the amygdala, PS rats should be pre-disposed to display increased fear-conditioned responses. As will be discussed below, alterations in other brain regions may also contribute to the enhanced freezing response. 
The observations regarding the importance of context in conditioning experiments for explicit cues led to a revision in the fear-potentiated startle methods. The training was conducted as before, using 2 training days with $0.5 \mathrm{~mA}$ of shock. However, during the testing session, the shock grids were removed and $70 \%$ ethanol was used to clean between test sessions creating differences in tactile and olfactory cues between the training and testing sessions. These simple changes resulted in a dramatic potentiation in the CON rats (Figure 18). Surprisingly, there was a smaller potentiation in the PS group indicated by the difference in responding to the light + noise trials. However, statistical analysis did not reveal a difference between the calculated difference scores (Figure 18). Additionally, the difference score was evaluated as a function of time in this experiment (Figure 18). Both groups demonstrated a significant decline in difference scores as the session progressed. This pattern resembles an extinction phenomenon and indicates that the rats learned the CS-US association because the association was less fear provoking later in the test session (Davis and Astrachan, 1978).

The null period responses recorded during this FPS experiment are shown in Figure 19. The findings demonstrate that the methodological changes were adequate to mask the training environment in terms of peak startle responding since neither group showed changes in these responses during the leader trials, as was found during the previous FPS experiments (Figure 15). However, during this experiment, null period responses were decreased during the testing trials when the CS was being presented to the rats, albeit to the same extent in either group. Although there was not a difference between the groups, this depression in null period responding is consistent with states of fear suppressing voluntary motor activity in rodents (Fanselow, 1991). 
It is important to note that the difference scores calculated in this experiment were not statistically different; however, the $\mathrm{CON}$ rats demonstrated much larger responses to the light + noise trials than the PS rats (Figure 18). It was expected that the PS rats would have a difference score of similar magnitude or greater than that of the CON rats. Since the PS rats did not demonstrate a large, positive difference score as expected, there is a possibility that the PS rats are not as fearful of the CS as the CON rats or perhaps they did not learn the CS-US association as readily as the CON rats. Two findings in the present work argue against these possibilities. First, the PS rats demonstrated enhanced behavioral inhibition to context after only one training trial (Figure 16) and to a light cue after two training trials (Figure 17). Secondly, the PS rats demonstrated an extinction phenomenon in the last FPS experiment proving that they did learn the association in this paradigm. Both of these findings indicate that PS rats are fully capable of learning fearful associations, even after only a single conditioning trial.

What, then, could explain the results? Davis has described a non-monotonic relationship with shock intensity used in training and difference scores (Davis and Astrachan, 1978). At the low end of the scale, low shock intensity produces low difference scores. As shock intensity increases the difference score will increase up to a certain point but then begin to decline as the shock intensity continues to increase. The $0.5 \mathrm{~mA}$ intensity used in these experiments was in the middle of the curve described by Davis and his colleagues. Thus, the suggestion is that PS rats experience shock differently and this shifts the non-monotonic relationship to the left in that group relative to the CON group. This suggestions fits the theory that PS rats are generally more fearful than CON rats. 
Davis and his colleagues have also shown that chemical lesions of the PAG restore potentiation to normal levels in rats trained at high shock intensities (Walker et al., 1997). A specific neural mechanism has been discovered that might explain these observations. Fendt and his colleagues have demonstrated that somatostatinergic projections from the periaqueductal gray (PAG), when activated, interfere with the generation of fearpotentiated startle responses (Figure 1) (Fendt et al., 1996). Importantly, baseline startle responding is not hindered by this action of somatostatin. Thus, the fear-potentiated startle response is selectively interupted by the PAG

It is important to mention that there is a discrepancy between these theories proposed by Davis and Fendt regarding this action of the PAG. Fendt and colleagues demonstrated, via tract-tracing analysis, that the lateral and ventral PAG supplies the somstatinergic projections to the RPC (Fendt et al, 1996). On the other hand, Davis and his colleagues have demonstrated, by chemical lesion studies, that it is the dorsal-lateral PAG (Walker et al, 1997). The two studies are not incompatible if one considers that the overlap between the two studies involves the lateral aspect of the PAG. Nevertheless, the studies also suggest that other areas of the PAG, and probably other neurotransmitter systems, may also be involved in the inhibition of the potentiated startle.

As suggested above, it is possible that PS rats "experience" the footshock more intensely than the $\mathrm{CON}$ animals. And this results in a low difference score by recruiting the somatostatinergic-based suppressant activity of the PAG on fear-potentiated startle. This idea would also be consistent with the enhanced behavioral inhibition seen in the PS rats in this study (Figure 16). In other words, the PAG may be activated at lower shock intensities in the PS rats; thus, behavioral inhibition is increased and fear-potentiated startle 
is decreased. Nevertheless, shock-induced movement responses measured during training are not different between the groups and do not support the idea that the PS rats are reacting differently to the shock during the training (See results). However, as mentioned several times already, PS rats are known to have elevated corticosterone responses to shock challenge (Weinstock et al., 1998) (Takahashi and Kalin, 1991). Therefore the lack of a difference between the groups in terms of shock-induced movement during training does not preclude differences from being detected during the testing phase of an experiment since increased plasma corticosterone is associated with increased responding in behavioral indices of fear [(Campeau et al., 1997) (Cordero et al, 1998) and see also (Korte, 2001)].

The PAG, together with the amygdala, integrates a number of defensive responses to prepare an animal for conflict (Fanselow, 1991). Lesion studies and drug studies demonstrate the involvement of the PAG in generating the freezing response to conditioned aversive stimuli (Kim et al, 1993) (Fanselow, 1991). In this regard, enhanced activation of the PAG during fear conditioning may explain the increased behavioral inhibition to aversive contexts with the PS rats. In addition to the results presented here, Takahashi and co-workers provided the first evidence that PS rats do freeze more following acute footshock (Takahashi et al, 1992a). The evidence from the current studies implicating the PAG, together with previous findings showing enhanced CRHergic activity in the amygdala of PS rats, provides a plausible mechanism for the enhanced behavioral inhibition and a "depression" of the FPS response, both of which would be indicative of greater fear on the part of the PS rats.

Latency to peak response was the final parameter evaluated in the FPS experiments (Table 5). Generally speaking, FPS training resulted in faster latency times during the 
testing trials in both groups when compared to the baseline values. Latency times were depressed across both trial types and not just to the light + noise trials. This decrease is consistent with a yohimbine study on the effects of startle in humans, where that known anxiogenic drug decreased the latency times of the peak startle response (Morgan, III et al., 1993). The data obtained during the testing trials are also consistent with the general negative correlation of peak startle response and latency described earlier (Pilz and Schnitzler, 1996). Thus, decreases in latency times can be interpreted as an anxiogenic response in the FPS paradigm.

Furthermore, latencies were shorter during the leader trials of both experiments than the respective baseline values (Table 5). This pattern of responses suggests that the latency parameter is a more sensitive indicator of fear than peak startle responding, since the peak startle response during the leader trials is unchanged from baseline peak startle responses (See Figure 14 and results). Interestingly, the PS rats had shorter times during the leader trials of experiment B and this finding would be consistent with the proposition that the PS were more fearful, at least in the beginning of the test session, than the CON rats. Finally, this evidence, coupled with the lack of a difference in latency times between the noise alone and light + noise trials, is also more evidence suggesting that the latency parameter and the peak startle response are controlled by different mechanisms (See also the discussion above regarding 8-OH-DPAT and latency).

It is intriguing that, as with the vehicle responses in the 8-OH-DPAT study, the PS rats generally have shorter latencies than the $\mathrm{CON}$ rats in these experiments. However, the fear-conditioning training does not seem to enhance this phenomenon in the PS rats, since the relative differences were maintained. The difference in latency times are not large, but 
they do indicate that the PS rats have faster neurotransmission through the startle pathway. The exact mechanism for this effect of prenatal stress remains to elucidated.

In conclusion, PS rats demonstrate enhanced fear-conditioning in terms of behavioral inhibition and, perhaps, in terms of "depressed" FPS responding. Prenatal stress may affect the functioning of the PAG-amygdala system that can be detected in terms of: 1) dramatically elevated acoustic startle responding after four days of footshock-based training; 2) "suppressed" fear-potentiated startle responding under moderate training conditions; and 3) enhanced behavioral inhibition to context after only a brief aversive training session. Additionally, it appears that PS rats generally have shorter times to reach the peak startle response. Finally, in general, fear-conditioning decreases the latency of the peak startle response and may serve as a very sensitive indicator of fear.

\section{5-HT $T_{1 A}$ Receptor Binding and cAMP production}

Two considerations revealed the importance of measuring and comparing the number of 5- $\mathrm{HT}_{1 \mathrm{~A}}$ receptors between PS and CON rats. First, there was the observation that PS rats were more sensitive to the anxiogenic effects of 8-OH-DPAT in ASR testing. Changes in receptor density might provide a rationale for this observation. Second, there was the observation that PS rats were more sensitive to fear-conditioning, particularly with regard to context. As described in the introduction, the amygdala and the hippocampus play important roles in fear-conditioned responses [e.g. (Campeau and Davis, 1995) (Phillips and LeDoux, 1992)].

The density of 5- $\mathrm{HT}_{1 \mathrm{~A}}$ receptors in block dissections of the amygdala were found not to be different between the groups. However, density of $5-\mathrm{HT}_{1 \mathrm{~A}}$ receptors in block dissections of the hippocampus were found to be different between the groups, with the PS 
rats having roughly $30 \%$ fewer receptors. As a brief aside, it should be noted that the relative differences between the amygdala and the hippocampus as well as the calculated $\mathrm{K}_{\mathrm{D}}$ values agree with published reports (Chalmers and Watson, 1991) (Popova et al., 1998) (Nenonene et al., 1994).

The reason for decreased numbers of 5-HT ${ }_{1 \mathrm{~A}}$ receptors in the hippocampus of PS rats is unclear. Nevertheless, the literature offers some ideas on why this may be the case. As pointed out previously, PS rats have a dysregulated HPA axis that ultimately results in prolonged, higher levels of circulating plasma corticosterone. High levels of circulating corticosterone are damaging to the hippocampus and result in decreases in the number of synapses (McEwen, 1999). A recent report, by Hayashi et. al., has demonstrated that prenatal stress results in offspring with $32 \%$ fewer synaptic connections in the hippocampus (Hayashi et al, 1998). Consequently, it is reasonable to speculate that the decrease in 5- $\mathrm{HT}_{1 \mathrm{~A}}$ binding found in the hippocampus of PS rats is due to decreases in the number of synapses.

The involvement of the hippocampus in spatial learning is well documented. Lesions of the hippocampus impair the conditioned freezing response to contextual cues (Phillips and LeDoux, 1992) (Kim et al, 1993). It would appear that if there is damage to the hippocampus caused by excessive, stressed-induced corticosterone in PS rats, it does not impair fear-conditioned responses to context as indicated by the behavioral inhibition experiments (Figures 16 and 17). In support of this observation, Hayashi et. al. showed that, despite synaptic loss of $32 \%$ in the hippocampus, PS rats performed equally as well as the CON rats in spatial learning tasks (Hayashi et al, 1998). In fact, it might be hypothesized that the enhanced behavioral inhibition to context may be aided by synaptic 
loss in PS rats if those synapses contained 5- $\mathrm{HT}_{1 \mathrm{~A}}$ receptors and those receptors are involved in generating anxiolytic tone.

The $5-\mathrm{HT}_{1 \mathrm{~A}}$ system of the hippocampus has been shown to be involved in fearful behaviors. For example, Kostowski et. al. showed that a high dose of the $5-\mathrm{HT}_{1 \mathrm{~A}}$ partialagonist buspirone injected into the hippocampus increased exploratory behavior in the open field and the elevated plus maze, which are considered anxiolytic effects (Kostowski et al., 1989). Additionally, Steidl et. al. showed that activation of 5- $\mathrm{HT}_{1 \mathrm{~A}}$ receptors with $8-\mathrm{OH}-$ DPAT in the hippocampus, impaired fear-conditioned freezing in mice and this effect could be blocked by WAY-100635 (Stiedl et al., 2000). Given these reports, it could be posited that the deficit in 5- $\mathrm{HT}_{1 \mathrm{~A}}$ receptors in the hippocampus of PS rats contributes to the increased behavioral inhibition to context noted in Figure 16.

Not to be forgotten, cAMP was measured in the hippocampus and the amygdala under basal conditions as well as stimulation by $10 \mu \mathrm{M}$ Forskolin (Figure 22). No differences were found in either brain region or condition. This second messenger activates a variety of intracellular effectors, such as protein kinase A, that are critical for a variety of nervous system functions, including production of $\mathrm{CRH}$ in the hypothalamus and memory consolidation in fear-conditioning (Itoi et al., 1996) (Schafe and LeDoux, 2000). Interestingly, the basal values found in the amygdala were roughly 3 times that of that found in the hippocampus and might indicate that this second messanger is relatively more important in this brain region. Slight differences in the incubation times of the reactions only provide a partial explanation for this observation, since cAMP production increases linearly with reaction time (De Vivo and Maayani, 1986). 
In conclusion, prenatal stress decreases $5-\mathrm{HT}_{1 \mathrm{~A}}$ binding in the hippocampus but not in the amygdala. The reason is unclear, but it may be related to changes in synaptic density. A brief look at cAMP production demonstrated that there are not gross alterations in this second messenger system, however, this does not preclude differences from existing with the intracellular effectors such as CREB or PKA.

\section{Issues of Context and Specific Cues}

The data shown in the studies on fear-conditioning present an intriguing opportunity to discuss behavioral reactions to context and specific cues. As shown in Figures 16 and 17 , behavioral inhibition is a robust response in rodents presented with fear provoking stimuli, even with brief exposures to those stimuli. In fact, in the experiment where the rats were trained to freeze to a light cue, there was considerable freezing to the context as well as the light cue (Figure 17). This was in spite of tactile and olfactory changes in the conditioning chamber intended to disguise the training environment and maximize the freezing response to the light cue.

Behavioral inhibition was also detected in the FPS experiments in the PS rats by depressed null period responding. This indicated that, following training, the PS rats were freezing to the context where the training had occurred and this subsquently lead to the hypothesis regarding enhanced conditioned freezing. This is an important point, since the only other study to look at this issue in PS rats found no conditioning effect (See discussion above) (Takahashi et al, 1992a). The null period responses were also important in proving the effectiveness of the contextual changes implemented in the FPS experiment shown in Figure 18, since null period responding was unchanged during the leader trials and a 
difference score was calculated (Figure 19). That set of data helped demonstrate that the calculation of the difference score is remarkably sensitive to contextual changes.

Nonetheless, although null period responding is a quite objective measure of behavioral inhibition, it may only be a useful approximation for the assessment of freezing behavior. As mentioned previously, the null period is only a short interval of time relative to the test session and equipment design prevents lengthening that period. Secondly, due to the nature of acoustic startle testing, the rat is enclosed in a small area and not permitted much room to move. By their nature, these two points weaken the use of the null period response as an estimate of behavioral inhibition.

Peak startle responding itself is not affected by context to the same extent as freezing. Even four days of footshock based training does not elevate the peak startle responses during the leader trials to levels that are statistically different from baseline (Figure 14b). On the other hand, peak startle responding is responsive to a specific cue. General increases in startle responding were seen during the portions of the test when the light cue was presented to the rats after 2 days of training even though a positive difference score could not be calculated (Figure 14a). However, as noted above, contextual cues can "obscure" the calculation of a difference score, since seemingly small changes in context allowed the difference score to emerge (Figure 18). Thus, if changes in contextual cues allowed the calculation of a difference score, then the state of fear that allows the calculation of the difference score must be a fleeting entity.

The latency to peak startle response is also affected by fear conditioning and it may be the most sensitive indicator of fear recorded in the FPS studies (Table 5). Latency was decreased after training regardless of any methodological manipulations and there were 
differences in the degree of depression. First, in experiment A shown in Table 5, latency was decreased to same extent during leader and testing trials, suggesting an effect of context on this parameter because the decrease was seen prior to the presentation of the light cue. It is worth noting that the leader trial peak startle responses were not different from baseline peak startle responses in this experiment (Figure 14a). Secondly, the contextual changes implemented in the second FPS experiment did not totally leave leader trial latencies unchanged from the baseline latencies, even though peak startle responding was again unchanged during the leader trials compared to the baseline trials (Table 5 and results). Latency is also affected by the presence of a specific cue since, during experiment B, latencies were decreased even further during the testing trials. Finally, latency times appear to have a lower limit that was reached in both experiments during the testing trials since the times were remarkably similar (Table 5). In fact, during the testing trials differences were not detected between the two trial types used in the FPS tests (See results).

It is usually emphasized that the conditioned response obtained in FPS experiments is a "state of fear" and not simply the increase of peak startle responding in the presence of the light cue (Davis, 1990). Published evidence would describe this state of fear as occuring at a specific point in time that is confined to the period when the light cue is presented to the animal because peak startle responses are greatest during this time (Davis et al, 1989). Certainly, the data shown in Figure 18 support this notion, since difference scores were calculated using the peak startle responses. However, given the findings with the latency parameter as well as the null period responses during the testing trials of this experiment, the scope of what encompasses a "state of fear" must be expanded. In fact, the 
lack of differences between the trial types with the latency parameter and the null period responses demonstrates that the rats are not merely switching between a "state of fear" and a "state of non-fear" as the light is switched on and off. Following this logic then, one interpretation of the present data set is that, upon presentation of appropriate contextual cues, the rats enter a generalized state of fear regardless of how the peak startle response might be affected. If this proposition is true, then the potentiated startle response is not merely indicative of anticipatory anxiety, as has been proposed (Koch, 2000).

\section{Conclusion}

The data presented in this report demonstrate that changes produced by prenatal stress are quite varied and are likely to affect a variety of brain nuclei and neurotransmitter systems. These changes are evident in the behaviors assessed in this project. For example, PS rats are more sensitive than CON rats to the effects of 8-OH-DPAT on startle responding. Additionally, PS rats have shorter processing times through the acoustic startle pathway as indicated by faster latency times, although peak startle responding under baseline conditions is not usually different from that of the CON rats. PS rats demonstrate an enhanced behavioral inhibition to acute footshock as well as to contextual stimuli previously associated with footshock. And finally, PS rats may demonstrate greater fearconditioned responses in the FPS paradigm as evidenced by "suppressed" peak startle responses to the light + noise trials and faster latency times during the leader trials.

It is intriguing that a simple manipulation, such as a once daily saline injection, performed at a critical time of development can result in behavioral changes later in life. Even more intriguing is the idea that behaviors are selectively affected by such a 
manipulation. For example, exploratory behavior in a novel environment (e.g. estimated by numbers of rears) prior to footshock was similar between PS and CON rats. Yet the footshock elucidated rather clear differences in the behavior of the PS and CON rats. Likewise, peak startle responding is not usually different, but the latency to peak startle responding tends to be shorter in PS rats. These observations point to the plasticity of the central nervous system since there are unlikely to be gross differences in neural circuitry underlying these behaviors between the PS and CON rats.

A theme that emerged throughout the discussion was that the root of the behavioral differences between PS and CON rats is excessive exposure to corticosterone on the part of the PS rats, either during pregnancy or later in life because of the HPA axis dysregulation. As described in the introduction, corticosterone has widespread effects on cellular functions. If one couples this observation with the plethora of reports that describe the effects of prenatal stress on corticosterone secretion, either in the mother or the offspring, then this becomes a reasonable conclusion. However, corticosterone is only one of many stress-responsive hormones and it happens to be easily measured. Other stress-responsive systems have not received nearly the level of attention as has corticosterone. For example, vasopressin and oxytocin are released during stress and both are capable of affecting behavior (Young, 1999). Thus, it is unlikely that corticosterone is unilaterally causing the behavioral differences and keeps the field open for further exploration.

Several of the behaviors examined in this project relied on learning and memory in order to measure a response. The data show that the PS rats are more adept at learning fearful associations than the CON rats. A neural mechanism for supporting learning and memory that has been proposed is long-term potentiation, which is described as a 
strengthening of synaptic connections (Lynch et al., 1988). It has been shown to occur in the amygdala as a result of fear-conditioning (Rogan et al., 1997). And it has also been widely studied in the hippocampus [e.g. (Ikegaya et al., 1996) (Sarvey et al., 1989)]. Of course, as has been alluded to above, these two brain regions are integral to the behaviors of interest in this project. Additionally, given the results found in the behavioral inhibition experiments, the PAG should be considered a brain region where neural plasticity is occuring as a result of prenatal stress. It could be hypothesized that the long-term potentiation mechanism is altered by prenatal stress in brain regions that are known to be involved with specific behaviors.

An unmentioned rationale for examining a variety of behaviors was to find a behavior that was reliably different between PS and CON rats, yet would be easy to measure and interpret. One effect of prenatal stress stands out as particularly robust and deserving of future study. That is the effect of prenatal stress on acute footshock-induced freezing. Of all the behavioral differences reported in these studies, it produced the most dramatic differences between PS and CON rats. It has the advantage of being reproducible since this finding has been replicated in other experiments in the literature and in our laboratory (Skinner and Birkle, unpublished observations) (Takahashi et al, 1992a). Additionally, many rats can be evaluated in a single day of testing. It also has the advantage of being a single behavior which makes data interpretation much easier. In contrast, the defensive withdrawal test, although it is reproducible and a number of rats can be tested in a short period, there are a number of parameters that are measured which can make data interpretation difficult depending on the experimental circumstances [e.g. (Ward et al, 2000)]. Finally, the neuroanatomy of freezing behavior is well described; therefore, 
solid hypotheses can be derived and tested to determine why differences exist between PS and $\mathrm{CON}$ rats.

In closing, the rodent prenatal stress model provides a unique opportunity to explore the complex interactions in the brain. Future studies with this model will likely yield insight into the subtleties of nervous system functioning and the control of anxiety related behaviors. Understanding the cause of these differences should ultimately improve the understanding of psychological problems in people. 


\section{FUTURE DIRECTIONS}

The results presented in this manuscript raise many interesting questions about prenatal stress and provide opportunities for future experiments. A variety of broad subjects have been investigated in this project, some more thoroughly than others: parameters of development, chronic mild stress, functioning of the serotonergic system, elements of signal transduction and fear-conditioning. Although many avenues for future experiments have been explored in the discussion, a few seem to be particularly noteworthy.

1.) Are PS rats really more susceptible to the anhedonic effects of chronic mild stress? Future experiments should include a pre-stress baseline assessment of sucrose intake and a much "harsher" stressing regimen.

2.) Are PS rats really more fearful in the FPS paradigm? Future experiments should include several types of training regimens involving changing the number of CS-US pairings and the shock intensity. The purpose would be to elucidate the non-monotonic relationship in the difference scores described by Davis and his colleagues.

3.) What is the cause of the enhanced sensitivity to 8-OH-DPAT on the part of the PS rats? Future studies should include an evaluation of possible differences in metabolism as well as an attempt to block the rise in startle responding with WAY-100635 to ascertain if the effect of 8-OH-DPAT is actually due to $5-\mathrm{HT}_{1 \mathrm{~A}}$ receptors and not $5-\mathrm{HT}_{7}$ receptors.

4.) Extend the results reported in the section on signal transduction. For example, evaluated 5- $\mathrm{HT}_{1 \mathrm{~A}}$ binding in the DRN and look at the functional coupling of cAMP to 
receptors. Additionally, measuring intracellular effectors of cAMP, PKA and CREB, may yield explanations for the differences behaviors between PS and CON rats.

5.) Use the acute freezing response as a behavioral assay to evaluate differences between PS and CON rats with regard to specific brain nuclei. For example, use intraamygdalar or intra-PAG injections of various doses of D-Phe-CRF to determine the if the if previously reported the differences in CRHergic funtioning are contributing to the enhanced freezing to footshock.

6.) Can the prenatal stress paradigm provide a useful model for studying the role of corticosterone in processes related to learning and memory? For example, measure the long-term potentiation process in PS and CON rats. Can PS rats learn operant conditioning tasks as readily as they can learn fearful associations? 
William Clyde Griffin III

Center for Drug and Alcohol Programs

Institute of Pyschiatry, PO Box 250861

Medical University of South Carolina, Charleston, SC

Phone: 843-792-5951 Email: griffinw@musc.edu

Birth Date November 26, 1970

Present Position Post-doctoral Fellow

Center for Drug and Alcohol Programs

Institute of Pyschiatry

Medical University of South Carolina, Charleston, SC

Mentor: Larry Middaugh, PhD

\section{Education}

December 2001

May 1996

May 1993
Doctor of Philosophy, Pharmacology and Toxicology

School of Medicine, West Virginia University, Morgantown, WV

Mentor: Dale L. Birkle, PhD

Bachelor of Science degree, Pharmacy

School of Pharmacy, University of North Carolina, Chapel Hill, NC

Bachelor of Science degree, Biology

College of Arts and Sciences, University of North Carolina, Chapel Hill, NC

ASHP Accredited Pharmacy Practice Residency

July 1996- June 1997: Southeastern Regional Medical Center, Lumberton, NC

Timothy Giddens, MS, RPh, Residency Director

\section{Experience}

Jan.-Aug. 1995: Pharmacy Internship

Vaughn Independent Pharmacy, Carrboro, NC

William Edward Vaughn, RPh, Supervisor

Sept.1992-Jan.1995: Research Assistant

Lineberger Comprehensive Cancer Center, Chapel Hill, NC

H. Shelton Earp, MD, Supervisor

\section{Professional Licensure}

North Carolina Registered Pharmacist, License Number 13482

\section{Awards}

NIDA Institutional Post-Doctoral Fellowship, Medical University of South Carolina, 2001-2003

West Virginia University (WVU) School of Medicine (SoM) Competitive Stipend, 1999-2000

International Behavioral Neuroscience Society Travel Award 2000

NIH Institutional Pre-doctoral Fellowship, WVU SoM, 1997-1999

WVU SoM Academic Achievement Award (highest GPA) 1999

WVU Doctoral Student Travel Award 1999, 2000 and 2001

Summer Research Assistantship, Cancer Education Program, UNC Hospitals 1994

University of North Carolina Dean's List, 1992-1996

Eagle Scout 1985 


\title{
W.C. Griffin III, Page 2
}

\section{Professional Affiliations}

American Society for Pharmacology and Experimental Therapeutics, 2000 -present

Society for Neuroscience, 1999 -present

American Pharmaceutical Association, 1993 -present

\section{Service Activities}

ASPET Councilor, 2001 - present

WVU Graduate-Professional Student Council, Vice-Chair, 2000 - 2001

WVU Faculty Graduate Council, Ex-officio Member, 2000 - 2001

WVU SoM Distinguished Teacher Award Committee, Member, 2000 - 2001

Volunteer Pharmacist, The Care Clinic, Fayetteville, NC 1996-1997

\section{Professional Slide Presentations}

"Fear-Potentiated Startle in Prenatally Stressed Rats"

April 7, 2000; International Behavioral Neuroscience Society Meeting, Denver, Co.

"Evaluation of Antiemetic Therapy in an Outpatient Chemotherapy Population"

May 3, 1997; $28^{\text {th }}$ Annual Southeastern Regional Residency Conference, Athens, Ga.

\section{Lecture Presentations}

Autonomic Pharmacology, 3 hours

Spring 2000 and 2001

Pharmacology 160, Nursing and Dental Hygiene Pharmacology

\section{Manuscripts}

W.C. Griffin III and D.L. Birkle. "Prenatal Stress in Rats Alters Fear-Potentiated Startle and the

Freezing Response to Aversive Contexts" Physiology and Behavior (in preparation)

W.C. Griffin III and D.L. Birkle. "Latency to the Peak Startle Response is More Sensitive to Fear-

Conditioning than the Peak Startle Response" Physiology and Behavior (in preparation)

W.C. Griffin III, H.D. Skinner and D.L. Birkle. "Prenatal Stress in Rats Increases the Acoustic

Startle Response to 8-OH-DPAT" Pharmacology, Biochemistry and Behavior (in preparation)

William Griffin, June McDermott and Susan Gaylord. "Homeopathy in North Carolina."

Carolina Journal of Pharmacy. Vol. 78, No. 3, May/June 1998. Non-peer reviewed.

\author{
Abstracts \\ W.C. Griffin III and D.L. Birkle. "Prenatal Stress Alters Fear-Potentiated Startle but not the \\ Freezing Response to an Explicit Cue" Soc. Neurosci. Abstr. (submitted). \\ W.C. Griffin III and D.L. Birkle. "Evidence for Fear-Conditioning in Prenatally Stressed Rats" \\ Soc. Neurosci. Abstr. Vol. 26, Part 1, p. 488, 2000.
}




\section{W.C. Griffin III, Page 3}

W.C. Griffin III and D.L. Birkle. "Corticotropin Releasing Factor in Prenatally Stressed, Preweanling Rats.” Soc. Neurosci. Abstr., Vol. 25, Part 1, p.857, 1999.

D.A. White, W.C. Griffin III and D.L. Birkle. "The Differential Effects of Yohimbine and Idazoxan in Prenatally Stressed Rats Using Acoustic Startle are not mediated via $\alpha_{2}$ Adrenergic Neurotransmission.” Soc. Neurosci. Abstr., Vol. 25, Part 1, p.857, 1999

D. A. White, W.C. Griffin III, H.D. Skinner and D.L. Birkle. "Prenatal Stress Alters Serotonergic and Noradrenergic Functioning in Adult Rats." J. Neurochem. Vol. 72, S77, 1999 


\section{LITERATURE CITED}

American Psychological Association Diagnostic and Statistical Manual of Mental Disorders (DSM-IV) ,1995 American Psychological Association, Washington, D.C.

Aitken AR and Tork I (1988) Early development of serotonin-containing neurons and pathways as seen in wholemount preparations of the fetal rat brain. J.Comp.Neurol. 274:32-47.

Altman J and Bayer SA (1995) Atlas of Prenatal Rat Brain Development. CRC Press, Boca Raton.

Alvarez JC, Gluck N, Arnulf I, Quintin P, Leboyer M, Pecquery R, Launay JM, Perez-Diaz F, and Spreux-Varoquaux O (1999) Decreased platelet serotonin transporter sites and increased platelet inositol triphosphate levels in patients with unipolar depression: effects of clomipramine and fluoxetine. Clin.Pharmacol.Ther. 66:617-624.

Arthur JM, Casanas SJ, and Raymond JR (1993) Partial agonist properties of rauwolscine and yohimbine for the inhibition of adenylyl cyclase by recombinant human 5-HT $1 \mathrm{~A}$ receptors. Biochem.Pharmacol. 45:2337-2341.

Astrachan DI and Davis M (1981) Spinal modulation of the acoustic startle response: the role of norepinephrine, serotonin and dopamine. Brain Res. 206:223-228.

Astrachan DI, Davis M, and Gallager DW (1983) Behavior and binding: correlations between alpha 1-adrenergic stimulation of acoustic startle and alpha 1-adrenoceptor occupancy and number in rat lumbar spinal cord. Brain Res. 260:81-90.

Azmitia EC and McEwen BS (1974) Adrenalcortical influence on rat brain tryptophan hydroxylase activity. Brain Res. 78:291-302.

Baram TZ and Schultz L (1992) CRH gene expression in the fetal rat is not increased after pharmacological adrenalectomy. Neurosci.Lett. 142:215-218.

Barbazanges A, Piazza PV, Le Moal M, and Maccari S (1996) Maternal glucocorticoid secretion mediates long-term effects of prenatal stress. J.Neurosci. 16:3943-3949.

Battaglia G, Webster E, and De Souza EB (1987) Characterization of corticotropinreleasing factor receptor-mediated adenylate cyclase activity in the rat central nervous system. Synapse 1:572-581.

Bechara A, Damasio H, Damasio AR, and Lee GP (1999) Different contributions of the human amygdala and ventromedial prefrontal cortex to decision-making. J.Neurosci. 19:5473-5481.

Biegon A, Rainbow TC, and McEwen BS (1985) Corticosterone modulation of neurotransmitter receptors in rat hippocampus: a quantitative autoradiographic study. Brain Res. 332:309-314. 
Birnbaum SG and Davis M (1998) Modulation of the acoustic startle reflex by infusion of corticotropin-releasing hormone into the nucleus reticularis pontis caudalis. Brain Res.

782:318-323.

Bittencourt JC and Sawchenko P (2000) Do Centrally Administered Neuropeptides Access Cognate Receptors?: An Analysis in the Central Corticotropin-Releasing Factor System. J.Neurosci. 20:1142-1158.

Blanchard RJ and Blanchard DC (1969) Crouching as an Index of Fear. J.Comp.Physiol.Psychol. 67:370-375.

Blanchard RJ, Nikulina JN, Sakai RR, McKittrick C, McEwen B, and Blanchard DC (1998) Behavioral and endocrine change following chronic predatory stress. Physiol.Behav. 63:561-569.

Blanchard RJ, Shepherd JK, Armstrong J, Tsuda SF, and Blanchard DC (1993) An ethopharmacological analysis of the behavioral effects of 8-OH-DPAT.

Psychopharmacol.(Berl.) 112:55-63.

Bouwer C, Claassen J, Dinan TG, and Nemeroff CB (2000) Prednisone augmentation in treatment-resistant depression with fatigue and hypocortisolaemia: a case series. Depress.Anxiety 12:44-50.

Broekkamp C (1997) Predictive validity and the robustness criterion for animal models. Psychopharmacol.(Berl.) 134:341-343.

Brown JS, Kalish HI, and Farber IE (1951) Conditioned fear as revealed by magnitude of startle response to an auditory stimulus. J.Exp.Psychol. 41:317-328.

Butler PD, Susser ES, Brown AS, Kaufmann CA, and Gorman JM (1994) Prenatal nutritional deprivation as a risk factor in schizophrenia: Preclinical evidence.

Neuropsychopharmacol. 11:227-235.

Campeau S and Davis M (1995) Involvement of the central nucleus and basolateral complex of the amygdala in fear conditioning measured with fear-potentiated startle in rats trained concurrently with auditory and visual conditioned stimuli. J.Neurosci. 15:23012311.

Campeau S, Falls WA, Cullinan WE, Helmreich DL, Davis M, and Watson SJ (1997) Elicitation and reduction of fear: Behavioural and neuroendocrine indices and brain induction of the immediate-early gene c-fos. Neurosci. 78:1087-1104.

Campeau S, Liang KC, and Davis M (1990) Long-term retention of fear-potentiated startle following a short training session. Anim.Learn.Behav. 18:462-468.

Canger R, Battino D, Canevini MP, Fumarola C, Guidolin L, Vignoli A, Mamoli D, Palmieri C, Molteni F, Granata T, Hassibi P, Zamperini P, Pardini G, and Avanzini G 
(1999) Malformations in offspring of women with epilepsy: a prospective study. Epilepsia 40:1231-1236.

Chalmers DT, Lovenberg TW, and De Souza EB (1995) Localization of novel corticotropin-releasing factor receptor (CRF2) mRNA expression to specific subcortical nuclei in rat brain: comparison with CRF1 receptor mRNA expression. J.Neurosci. 15:6340-6350.

Chalmers DT and Watson SJ (1991) Comparative anatomical distribution of 5-HT $1 \mathrm{~A}$ receptor mRNA and 5- $\mathrm{HT}_{1 \mathrm{~A}}$ binding in rat brain - a combined in situ hybridisation/in vitro receptor autoradiographic study. Brain Res. 561:51-60.

Chaouloff F (1993) Physiopharmacological interactions between stress hormones and central serotonergic systems. Brain Res.Rev. 18:1-32.

Cheeta S, Ruigt G, van Proosdij J, and Willner P (1997) Changes in sleep architecture following chronic mild stress. Biol.Psychiatry 41:419-427.

Chen R, Lewis KA, Perrin MH, and Vale WW (1993) Expression cloning of a human corticotropin-releasing-factor receptor. Proc.Natl.Acad.Sci.USA 90:8967-8971.

Chopin P and Briley M (1987) Animal models of anxiety: The effect of compounds that modify 5-HT neurotransmission. Trends Pharmacol.Sci. 8:383-388.

Clarke A and Schneider M (1993) Prenatal stress has long-term effects on behavioral responses to stress in juvenile rhesus monkeys. Dev.Psychobiol. 26:293-304.

Clarke A, Wittwer D, Abbott D, and Schneider M (1994) Long-term effects of prenatal stress on HPA axis activity in juvenile rhesus monkeys. Dev.Psychobiol. 27:257-269.

Collinson N and Dawson GR (1997) On the elevated plus maze the anxiolytic like effects of the 5-HT $\mathrm{H}_{1 \mathrm{~A}}$ agonist, 8-OH-DPAT, but not the anxiogenic- like effects of the 5-HT $\mathrm{H}_{1 \mathrm{~A}}$ partial agonist, buspirone, are blocked by the 5-HT $\mathrm{H}_{1 \mathrm{~A}}$ antagonist, WAY 100635.

Psychopharmacol.(Berl.) 132:35-43.

Cordero MI, Merino JJ, and Sandi C (1998) Correlational relationship between shock intensity and corticosterone secretion on the establishment and subsequent expression of contextual fear conditioning. Behav.Neurosci. 112:885-891.

Cratty MS, Ward HE, Johnson EA, Azzaro AJ, and Birkle DL (1995) Prenatal stress increases corticotropin-releasing factor $(\mathrm{CRF})$ content and release in rat amygdala minces. Brain Res. 675:297-302.

Cruz AP, Bueno JL, and Graeff FG (1993) Influence of stimulus preexposure and of context familiarity on conditioned freezing behavior. Braz.J.Med.Biol.Res. 26:615-621. 
Cummings S, Elde R, Ells J, and Lindall A (1983) Corticotropin-releasing factor immunoreactivity is widely distributed within the central nervous system of the rat: An immunhistochemical study. J.Neurosci. 3:1355-1368.

D'Aquila PS, Newton J, and Willner P (1997) Diurnal variation in the effect of chronic mild stress on sucrose intake and preference. Physiol.Behav. 62:421-426.

Davis M (1984) The Mammalian Startle Response, in Neural Mechanisms of Startle Behavior (Eaton RC ed) pp 287-351, Plenum Press, New York.

Davis M (1986) Pharmacological and anatomical analysis of fear conditioning using the fear-potentiated startle paradigm. Behav.Neurosci. 100:814-824.

Davis M (1990) Animal models of anxiety based on classical conditioning: The conditioned emotional response (CER) and the fear- potentiated startle effect.

Pharmacol.Therap. $47: 147-165$.

Davis M and Astrachan DI (1978) Conditioned fear and startle magnitude: effects of different footshock or backshock intensities used in training. J.Exp.Psychol.,Anim Behav.Process. 4:95-103.

Davis M and Astrachan DI (1981) Spinal modulation of acoustic startle: opposite effects of clonidine and d-amphetamine. Psychopharmacol.(Berl.) 75:219-225.

Davis M, Cassella JV, and Kehne JH (1999) Serotonin does not Mediate Anxiolytic Effects of Buspirone in the fear-potentiated Startle Paradigm: Comparison with 8-OH-DPAT and Ipsapirone. $x x$.

Davis M, Gendelman DS, Tischler MD, and Gendelman PM (1982) A primary acoustic startle circuit: lesions and stimulation studies. J.Neurosci. 6:791-805.

Davis M, Hitchcock JM, and Rosen JB (1987) Anxiety and the amygdala: pharmacological and anatomical analysis of the fear potentiated startle paradigm, in The psychology of learning and motivation (Bower GH ed) pp 263-292, Academic Press, New York.

Davis M, Schlesinger LS, and Sorenson CA (1989) Temporal specificity of fearconditioning: effects of different conditioned stimulus-unconditioned stimulus intervals on the fear-potentiated startle effect. J.Exp.Psychol.,Anim Behav.Process. 15:295-310.

Davis M and Sheard MH (1974) Habituation and Sensitization of the Rat Startle Response: Effects of Raphe Lesions. Physiol.Behav. 12:425-431.

De Almeida RMM and Lucion AB (1997) 8-OH-DPAT in the median raphe, dorsal periaqueductal gray and corticomedial amygdala nucleus decreases, but in the medial septal area it can increase maternal aggressive behavior in rats. Psychopharmacol.(Berl.) 134:392-400. 
De Bellis MD, Gold PW, Geracioti TD, Jr., Listwak SJ, and Kling MA (1993) Association of fluoxetine treatment with reductions in CSF concentrations of corticotropin-releasing hormone and arginine vasopressin in patients with major depression. Am.J.Psychiatry 150:656-657.

De Souza EB (1987) Corticotrophin-releasing factor receptors in the rat central nervous system: Characterization and regional distribution. J.Neurosci. 7:88-100.

De Vivo M and Maayani S (1986) Characterization of the 5-hydroxytryptamine1a receptormediated inhibition of forskolin-stimulated adenylate cyclase activity in guinea pig and rat hippocampal membranes. J.Pharmacol.Exp.Ther. 238:248-253.

Deak T, Nguyen KT, Ehrlich AL, Watkins LR, Spencer RL, Maier SF, Licinio J, Wong ML, Chrousos GP, Webster E, and Gold PW (1999) The impact of the nonpeptide corticotropin-releasing hormone antagonist antalarmin on behavioral and endocrine responses to stress. Endocrinology 140:79-86.

DeSouza EB (1995) Corticotropin-releasing factor receptors: physiology, pharmacology, biochemistry and role in central nervous system and immune disorders. pne 20:789-819.

Dunn AJ and Berridge CW (1990) Physiological and behavioral responses to corticotropinreleasing factor administration: Is CRF a mediator of anxiety or stress responses? Brain Res.Rev. 15:71-100.

Eghbal-Ahmadi M, Avishai-Eliner S, Hatalski CG, and Baram TZ (1999) Differential regulation of the expression of corticotropin-releasing factor receptor type $2\left(\mathrm{CRF}_{2}\right)$ in hypothalamus and amygdala of immature rat by sensory input and food intake. J.Neurosci. 19:3982-3991.

Eghbal-Ahmadi M, Hatalski CG, Lovenberg TW, Avishai-Eliner S, Chalmers DT, and Baram TZ (1998) The developmental profile of the corticotropin releasing factor receptor $\left(\mathrm{CRF}_{2}\right)$ in rat brain predicts distinct age-specific functions. Dev.Brain Res. 107:81-90.

Elkabir DR, Wyatt ME, Vellucci SV, and Herbert J (1990) The effects of separate or combined infusions of corticotrophin-releasing factor and vasopressin either intraventricularly or into the amygdala on aggressive and investigative behaviour in the rat. Regul.Pept. 28:199-214.

Engelmann M, Thrivikraman KV, Su Y, Nemeroff CB, Montkowski A, Landgraf R, Holsboer F, and Plotsky PM (1996) Endocrine and behavioral effects of airpuff-startle in rats. pne 21:391-400.

Fanselow MS (1991) The midbrain periaqueductal gray as a coordinator of action in repsonse to fear and anxiety, in The Midbrain Periaqueductal Gray Matter (DePaulis A and Bandler R eds) pp 151-173, Plenum Press, New York and London.

Fanselow MS and Helmstetter FJ (1988) Conditioned Analgesia, Defensive Freezing, and Benzodiazepines. Behav.Neurosci. 102:233-243. 
Fendt M and Fanselow MS (1999) The neuroanatomical and neurochemical basis of conditioned fear. Neurosci.Biobehav.Rev. 23:743-760.

Fendt M, Koch M, and Schnitzler H-U (1996) Somatostatin in the pontine reticular formation modulates fear potentiation of the acoustic startle response: an anatomical, electrophysiological, and behavioral study. J.Neurosci. 16:3097-3103.

Fendt M, Koch M, and Schnitzler HU (1997) Corticotropin-releasing factor in the caudal pontine reticular nucleus mediates the expression of fear-potentiated startle in the rat. Eur.J.Neurosci. 9:299-305.

Figueras G, Perez V, San Martino O, Alvarez E, and Artigas F (1999) Pretreatment platelet 5-HT concentration predicts the short-term response to paroxetine in major depression. Grupo de Trastornos Afectivos. Biol.Psychiatry 46:518-524.

File SE, Gonzalez LE, and Andrews N (1996) Comparative study of pre- and postsynaptic $5-\mathrm{HT}_{1 \mathrm{~A}}$ receptor modulation of anxiety in two ethological animal tests. J.Neurosci. 16:4810-4815.

Fisher LA (1989) Corticotropin-releasing factor: endocrine and autonomic intergration of responses to stress. Trends Pharmacol.Sci. 10:189-193.

Fletcher A, Forster EA, Bill DJ, Brown G, Cliffe IA, Hartley JE, Jones DE, McLenachan A, Stanhope KJ, Critchley DJ, Childs KJ, Middlefell VC, Lanfumey L, Corradetti R, Laporte AM, Gozlan H, Hamon M, and Dourish CT (1996) Electrophysiological, biochemical, neurohormonal and behavioural studies with WAY-100635, a potent, selective and silent 5-HT1A receptor antagonist. Behav.Brain Res. 73:337-353.

Forbes NF, Stewart CA, Matthews K, and Reid IC (1996) Chronic mild stress and sucrose consumption: validity as a model of depression. Physiol.Behav. 60:1481-1484.

Forster EA, Cliffe IA, Bill DJ, Dover GM, Jones D, Reilly Y, and Fletcher A (1995) A pharmacological profile of the selective silent 5-HT1A receptor antagonist, WAY-100635. Eur.J.Pharmacol. 281:81-88.

Fride E, Dan Y, Feldon J, Halevy G, and Weinstock M (1986) Effects of prenatal stress on vulnerability to stress in prepubertal and adult rats. Physiol.Behav. 37:681-687.

Fride E and Weinstock M (1988) Prenatal stress increases anxiety-related behavior and alters cerebral lateralization of dopamine activity. Life Sci. 42:1059-1065.

Gilman AG, Rall TW, Nies AS, and Taylor P (1990) Goodman and Gilman's The Pharmacological Basis of Therapeutics. Pergamon Press, New York.

Glowinski J and Iversen LL (1966) Regional studies of catecholamines in the rat brain: I. The disposition of $\left[{ }^{3} \mathrm{H}\right]$ norepinephrine, $\left[{ }^{3} \mathrm{H}\right]$ dopamine, and $\left[{ }^{3} \mathrm{H}\right]$ dopa in various regions of the brain. J.Neurochem. 13:655-669. 
Gozlan H, Thibault S, Laporte AM, Lima L, and Hamon M (1995) The selective 5-HT1A antagonist radioligand [3H]WAY 100635 labels both G-protein-coupled and free 5-HT1A receptors in rat brain membranes. Eur.J.Pharmacol. 288:173-186.

Graeff FG, Guimaraes FS, De Andrade TGCS, and Deakin JFW (1996) Role of 5-HT in stress, anxiety, and depression. Pharmacol.Biochem.Behav. 54:129-141.

Grillon C, Pellowski M, Merikangas KR, and Davis M (1997) Darkness facilitates the acoustic startle reflex in humans. Biol.Psychiatry 42:453-460.

Hall MD, El Mestikawy S, Emerit MB, Pichat L, Hamon M, and Gozlan H (1985) [3H]8hydroxy-2-(di-n-propylamino)tetralin binding to pre- and postsynaptic 5hydroxytryptamine sites in various regions of the rat brain. J.Neurochem. 44:1685-1696.

Handley SL (1995) 5-hydroxytryptamine pathways in anxiety and its treatment. Pharmacol.Ther. 66:103-148.

Handley SL and McBlane JW (1993) 5HT Drugs in Animal Models of Anxiety. Psychopharmacol.(Berl.) 112:13-20.

Harris RB, Zhou J, Youngblood BD, Smagin GN, and Ryan DH (1997) Failure to change exploration or saccharin preference in rats exposed to chronic mild stress. Physiol.Behav. 63:91-100.

Hayashi A, Nagaoka M, Yamada K, Ichitani Y, Miake Y, and Okado N (1998) Maternal stress induces synaptic loss and developmental disabilities of offspring. Int.J.Dev.Neurosci. $16: 209-216$.

Heaton MB, Paiva M, Swanson DJ, and Walker DW (1995) Alterations in responsiveness to ethanol and neurotrophic substances in fetal septohippocampal neurons following chronic prenatal ethanol exposure. Dev.Brain Res. 85:1-13.

Helmstetter FJ (1993) Stress-induced hypoalgesia and defensive freezing are attenuated by application of diazepam to the amygdala. Pharmacol.Biochem.Behav. 44:433-438.

Hepper PG (1995) Human fetal behaviour and maternal cocaine use: A longitudinal study. Neurotoxicology 16:139-144.

Hoyer D, Clarke DE, Fozard JR, Hartig PR, Martin GR, Mylecharane EJ, Saxena PR, and Humphrey PP (1994) International Union of Pharmacology classification of receptors for 5-hydroxytryptamine (Serotonin). Pharmacol.Rev. 46:157-203.

Hoyer D, Pazos A, Probst A, and Palacios JM (1986) Serotonin receptors in the human brain. I. Characterization and autoradiographic localization of 5-HT1A recognition sites. Apparent absence of 5-HT1B recognition sites. Brain Res. 376:85-96.

Huttunen MO and Niskanen P (1978) Prenatal loss of father and psychiatric disorders. Arch.Gen.Psychiatry 35:429-431. 
Ikegaya Y, Saito H, and Abe K (1996) The basomedial and basolateral amygdaloid nuclei contribute to the induction of long-term potentiation in the dentate gyrus in vivo.

Eur.J.Neurosci. 8:1833-1839.

Inoue T, Tsuchiya K, and Koyama T (1996) Serotonergic Activation Reduces Defensive Freezing in the Conditioned Fear Paradigm. Pharmacol.Biochem.Behav. 53:825-831.

Itoi K, Horiba N, Tozawa F, Sakai Y, Sakai K, Abe K, Demura H, and Suda T (1996) Major role of 3',5'-cyclic adenosine monophosphate-dependent protein kinase A pathway in corticotropin-releasing factor gene expression in the rat hypothalamus in vivo.

Endocrinology 137:2389-2396.

Jiang HK, Wang JY, and Lin JC (2000) The central mechanism of hypothalamic-pituitaryadrenocortical system hyperfunction in depressed patients. Psychiatry Clin.Neurosci. 54:227-234.

Joordens RJ, Hijzen TH, and Olivier B (1998) The effects of 5-HT1A receptor agonists, 5HT1A receptor antagonists and their interaction on the fear-potentiated startle response. Psychopharmacol.(Berl.) 139:383-390.

Joordens RJE, Hijzen TH, Peeters BWMM, and Olivier B (1997) Control conditions in the fear-potentiated startle response paradigm. Neuroreport 8:1031-1034.

Kalin NH, Shelton SE, Rickman M, and Davidson RJ (1998) Individual Differences in Freezing and Cortisol in Infant and Mother Rhesus Monkeys. Behav.Neurosci. 112:251254.

Kalin NH, Sherman JE, and Takahashi LK (1988) Antagonism of endogenous CRH systems attenuates stress-induced freezing behavior in rats. Brain Res. 457:130-135.

Kalin NH and Takahashi LK (1990) Fear-motivated behavior induced by prior shock experience is mediated by corticotropin-releasing hormone systems. Brain Res. 509:80-84.

Kasckow JW, Regmi A, Gill PS, Parkes DG, and Geracioti TD (1997) Regulation of corticotropin-releasing factor (CRF) messenger ribonucleic acid and CRF peptide in the amygdala: Studies in primary amygdalar cultures. Endocrinology 138:4774-4782.

Keshet GI and Weinstock M (1995) Maternal naltrexone prevents morphological and behavioral alterations induced in rats by prenatal stress. Pharmacol.Biochem.Behav. 50:413-419.

Kessler RC (1997) The effects of stressful life events on depression. Ann.Rev.Psychol. 48:191-214.

Kim JJ, Rison RA, and Fanselow MS (1993) Effects of Amygdala, Hippocampus, and Periaqueductal Gray Lesions on Short- and Long-Term Contextual Fear. Behav.Neurosci. 107:1093-1098. 
Kinsley C and Svare B (1987) Genotype modulates prenatal stress effects on aggression in male and female mice. Behav.Neural Biol. 47:138-150.

Koch M (2000) The Neurobiology of Startle. Prog.Neurobiol. 59:107-128.

Koch M, Schmid A, and Schnitzler HU (1996) Pleasure-attenuation of startle is disrupted by lesions of the nucleus accumbens. Neuroreport 7:1442-1446.

Koehl M, Darnaudéry M, Dulluc J, Van Reeth O, Le Moal M, and Maccari S (1999)

Prenatal stress alters circadian activity of hypothalamo-pituitary-adrenal axis and hippocampal corticosteroid receptors in adult rats of both gender. J.Neurobiol. 40:302-315.

Koob G and Heinrichs SC (1999) A Role for Corticotropin Releasing Factor and Urocortin in Behavioral Responses to Stressors. Brain Res. 848:141-152.

Korte SM (2001) Corticosteroids in relation to fear, anxiety and psychopathology. Neurosci.Biobehav.Rev. 25:117-142.

Kostich WA, Chen AR, Sperle K, and Largent BL (1998) Molecular identification and analysis of a novel human corticotropin-releasing factor $(\mathrm{CRF})$ receptor: The $\mathrm{CRF}_{2 \text { gamma }}$ receptor. Mol.Endocrinol. 12:1077-1085.

Kostowski W, Plaznik A, and Stefanski R (1989) Intra-hippocampal buspirone in animal models of anxiety. Eur.J.Pharmacol. 168:393-396.

Koyama S, Kubo C, Rhee JS, and Akaike N (1999) Presynaptic serotonergic inhibition of GABAergic synaptic transmission in mechanically dissociated rat basolateral amygdala neurons. J.Physiol. 518 ( Pt 2):525-538.

Krebs H, Macht M, Weyers P, Weijers HG, and Janke W (1996) Effects of stressful noise on eating and non-eating behavior in rats. Appetite 26:193-202.

Krebs H, Weyers P, Macht M, Weijers HG, and Janke W (1997) Scanning behavior of rats during eating under stressful noise. Physiol.Behav. 62:151-154.

Laaris N, Haj-Dahmane S, Hamon M, and Lanfumey L (1995) Glucocorticoid receptormediated inhibition by corticosterone of 5-HT1A autoreceptor functioning in the rat dorsal raphe nucleus. Neuropharmacology 34:1201-1210.

Landis C and Hunt W (1939) The Startle Pattern. Farrar and Rinehart, New York.

Lattimer N, McAdams RP, Rhodes KF, Sharma S, Turner SJ, and Waterfall JF (1984) Alpha $_{2}$-adrenoceptor antagonism and other pharmacological antagonist properties of some substituted benzoquinolizines and yohimbine in vitro. N-S Arch.Pharmacol. 327:312-318.

LeDoux JE, Iwata J, Cicchetti P, and Reis DJ (1988) Different projections of the central amygdaloid nucleus mediate autonomic and behavioral correlates of conditioned fear. J.Neurosci. 8:2517-2529. 
Lee S and Rivier C (1994) Prenatal alcohol exposure alters the hypothalamic-pituitaryadrenal axis response of immature offspring to interleukin- 1: Is nitric oxide involved. Alcoholism (NY) 18:1242-1247.

Lee Y, Lopez DE, Meloni EG, and Davis M (1996) A Primary Acoustic Startle Pathway: Obligatory Role of Cochlear Root Neurons and the Nucleus Reticularis Pontis Caudalis. J.Neurosci. 16:3775-3789.

Lee Y, Schulkin J, and Davis M (1994) Effect of corticosterone on the enhancement of the acoustic startle reflex by corticotropin releasing factor (CRF). Brain Res. 666:93-98.

Lee YL and Davis M (1997) Role of the hippocampus, the bed nucleus of the stria terminalis, and the amygdala in the excitatory effect of corticotropin- releasing hormone on the acoustic startle reflex. J.Neurosci. 17:6434-6446.

Leitner DS, Powers AS, and Hoffman HS (1980) The neural substrate of the startle response. Physiol.Behav. 25:291-297.

Liang KC and Lee EHY (1988) Intra-amygdala injections of corticotropin-releasing factor facilitate inhibitory avoidance learning and reduce exploratory behavior in rats. Psychopharmacol.(Berl.) 96:232-236.

Liang KC, Melia KR, Miserendino MJD, Falls WA, Campeau S, and Davis M (1992) Corticotropin-releasing factor: Long-lasting facilitation of the acoustic startle reflex. J.Neurosci. 12:2303-2312.

Lightman SL and Harbuz MS (1993) Expression of corticotropin-releasing factor mRNA in response to stress, in Corticotropin-Releasing Factor (Ciba Foundation Symposium 172) (Chadwick DJ, Marsh J, and Ackrill K eds) pp 173-198, Wiley, Chichester.

Lovenberg TW, Baron BM, de Lecea L, Miller JD, Prosser RA, Rea MA, Foye PE, Racke M, Slone AL, and Siegel BW (1993) A novel adenylyl cyclase-activating serotonin receptor (5-HT7) implicated in the regulation of mammalian circadian rhythms. Neuron 11:449-458.

Lovenberg TW, Chalmers DT, Liu CG, and De Souza EB (1995a) $\mathrm{CRF}_{2 \alpha}$ and $\mathrm{CRF}_{2 \beta}$ receptor mRNAs are differentially distributed between the rat central nervous system and peripheral tissues. Endocrinology 136:4139-4142.

Lovenberg TW, Liaw CW, Grigoriadis DE, Clevenger W, Chalmers DT, De Souza EB, and Oltersdorf T (1995b) Cloning and characterization of a functionally distinct corticotropinreleasing factor receptor subtype from rat brain. Proc.Natl.Acad.Sci.USA 92:836-840.

Lowry OH, Rosenbrough NJ, Farr AL, and Randall RJ (1951) Protein measurement with the Folin phenol reagent. J.Biol.Chem. 193:265-275.

Lubow R.E. (1989) Latent Inhibition and Conditioned Attention Theory. Cambridge University Press, New York. 
Lutz-Bucher B, Felix JM, and Koch B (1991) Activation of protein kinase C differentially regulates corticotropin-releasing factor-stimulated peptide secretion and cyclic AMP formation of intermediate and anterior pituitary cells in culture. Peptides 11:1183-1189.

Lynch G, Muller D, Seubert P, and Larson J (1988) Long-term potentiation: Persisting problems and recent results. Brain Res.Bull. 21:363-372.

Makino S, Shibasaki T, Yamauchi N, Nishioka T, Mimoto T, Wakabayashi I, Gold PW, and Hashimoto K (1999) Psychological stress increased corticotropin-releasing hormone mRNA and content in the central nucleus of the amygdala but not in the hypothalamic paraventricular nucleus in the rat. Brain Res. 850:136-143.

Malkoski SP, Handanos CM, and Dorin RI (1997) Localization of a negative glucocorticoid response element of the human corticotropin releasing hormone gene. Mol.Cell.Endocrinol. 127:189-199.

Mann JJ, Huang YY, Underwood MD, Kassir SA, Oppenheim S, Kelly TM, Dwork AJ, and Arango V (2000) A serotonin transporter gene promoter polymorphism (5-HTTLPR) and prefrontal cortical binding in major depression and suicide. Arch.Gen.Psychiatry 57:729-738.

Mason JP, Dring LG, and Caldwell J (1995) Pharmacokinetics of the 5hydroxytryptamine1A agonist 8-hydroxy-2-(N,N-di-n-propylamino)tetralin (8-OHDPAT) in the rat after intravenous and oral administration. Xenobiotica 25:1371-1380.

Matthews K, Forbes N, and Reid IC (1995) Sucrose consumption as an hedonic measure following chronic unpredictable mild stress. Physiol.Behav. 57:241-248.

McEwen BS (1999) Stress and hippocampal plasticity. Ann.Rev.Neurosci. 22:105-122.

McNish KA, Gewirtz JC, and Davis M (1997) Evidence of contextual fear after lesions of the hippocampus: A disruption of freezing but not fear-potentiated startle. J.Neurosci. 17:9353-9360.

Meijer A (1985) Child psychiatric sequelae of maternal war stress. Acta Psychiat.Scand. 72:505-511.

Meijer OC, Williamson A, Dallman MF, and Pearce D (2000) Transcriptional repression of the 5-HT1A receptor promoter by corticosterone via mineralocorticoid receptors depends on the cellular context. J.Neuroendocrinol. 12:245-254.

Meyer JS (1985) Biochemical effects of corticosteroids on neural tissues. Physiol.Rev. 65:946-1020.

Miyata I, Shiota C, Ikeda Y, Oshida Y, Chaki S, Okuyama S, and Inagami T (1999) Cloning and characterization of a short variant of the corticotropin-releasing factor receptor subtype from rat amygdala. Biochem.Biophys.Res.Commun. 256:692-696. 
Monleon S, D'Aquila P, Parra A, Simon VM, Brain PF, and Willner P (1995) Attenuation of sucrose consumption in mice by chronic mild stress and its restoration by imipramine. Psychopharmacol.(Berl.) 117:453-457.

Morgan CA, III, Grillon C, Southwick SM, Davis M, and Charney DS (1995) Fearpotentiated startle in posttraumatic stress disorder. Biol.Psychiatry 38:378-385.

Morgan CA, III, Southwick SM, Grillon C, Davis M, Krystal JH, and Charney DS (1993) Yohimbine-facilitated acoustic startle reflex in humans. Psychopharmacol.(Berl.) 110:342346.

Mork A and Geisler A (1990) 5-Hydroxytryptamine receptor agonists influence calciumstimulated adenylate cyclase activity in the cerebral cortex and hippocampus of the rat. Eur.J.Pharmacol. 175:237-244.

Mundey MK, Fletcher A, and Marsden CA (1996) Effects of 8-OHDPAT and 5-HT1A antagonists WAY100135 and WAY100635, on guinea-pig behaviour and dorsal raphe 5HT neurone firing. Br.J.Pharmacol. 117:750-756.

Nancy M.Sherwood and Paola S.Timiras (1970) A Stereotaxic Atlas of the Developing Rat Brain. University of California Press, Berkeley.

Nenonene EK, Carli RM, Grondin L, and Reader TA (1994) Heterogeneity of cortical and hippocampal 5-HT1A receptors: a reappraisal of homogenate binding with 8$[3 \mathrm{H}]$ hydroxydipropylaminotetralin. J.Neurochem. 62:1822-1834.

Newton RW and Hunt LP (1984) Pyschosocial stress in pregnancy and its relation to low birth weight. Brit.Med.J. 288:1191-1194.

Ogilvie J and Clarke RW (1998) Effect of RX 821002 at 5-HT ${ }_{1 \mathrm{~A}}$-receptors in rabbit spinal cord in vivo. Br.J.Pharmacol. 123:1138-1142.

Okuhara DY and Beck SG (1998) Corticosteroids alter 5-hydroxytryptamine1A receptoreffector pathway in hippocampal subfield CA3 pyramidal cells. J.Pharmacol.Exp.Ther. 284:1227-1233.

Owens MJ and Nemeroff CB (1991) Physiology and pharmacology of corticotropin releasing factor. Pharmacol.Rev. 43:425-473.

Palacios JM, Waeber C, Hoyer D, and Mengod G (1990) Distribution of Serotonin Receptors. Ann.N.Y.Acad.Sci. 600:36-52.

Palkovits M, Brownstein MJ, and Vale W (1985) Distribution of corticotropin-releasing factor in rat brain. Fed.Proc. 44:215-219.

Papp M, Moryl E, and Willner P (1996) Pharmacological validation of the chronic mild stress model of depression. Eur.J.Pharmacol. 296:129-136. 
Parks CL, Robinson PS, Sibille E, Shenk T, and Toth M (1998) Increased anxiety of mice lacking the serotonin ${ }_{1 \mathrm{~A}}$ receptor. Proc.Natl.Acad.Sci.USA 95:10734-10739.

Pasamanick B, Rogers ME, and Lilienfeld AM (1956) Pregnancy Experience and the development of behavior disorder in children. Am.J.Psychiatry 112:613-618.

Pauwels PJ (2000) Diverse signalling by 5-hydroxytryptamine (5-HT) receptors. Biochem.Pharmacol. 60:1743-1750.

Paxinos G and Watson C (1982) The Rat Brain in Stereotaxic Coordinates. Academic Press, New York.

Pelton GH, Lee YL, and Davis M (1997) Repeated stress, like vasopressin, sensitizes the excitatory effects of corticotropin releasing factor on the acoustic startle reflex. Brain Res. 778:381-387.

Peters DAV (1982) Prenatal stress: Effects on brain biogenic amine and plasma corticosterone levels. Pharmacol.Biochem.Behav. 17:721-725.

Peters DAV (1986a) Prenatal stress increases the behavioral response to serotonin agonists and alters open field behavior in the rat. Pharmacol.Biochem.Behav. 25:873-877.

Peters DAV (1986b) Prenatal stress: effect of development of rat brain serotonergic neurons. Pharmacol.Biochem.Behav. 24:1377-1382.

Peters DAV (1989) Effects of maternal stress during different gestational periods on the serotonergic system in adult rat offspring. Pharmacol.Biochem.Behav. 31:839-843.

Phillips RG and LeDoux JE (1992) Differential contribution of amygdala and hippocampus to cued and contextual fear conditioning. Behav.Neurosci. 106:274-285.

Picazo O, López-Rubalcava C, and Fernández-Guasti A (1995) Anxiolytic effect of the 5$\mathrm{HT}_{1 \mathrm{~A}}$ compounds 8-hydroxy-2- (di-n-propylamino) tetralin and ipsapirone in the social interaction paradigm: Evidence of a presynaptic action. Brain Res.Bull. 37:169-175.

Pilz PKD and Schnitzler HU (1996) Habituation and sensitization of the acoustic startle response in rats: amplitude, threshold, and latency measures. Neurobiol.Learn.Mem. 66:6779.

Plappert CF, Pilz PKD, and Schnitzler H-U (1993) Acoustic startle response and habituation in freezing and nonfreezing rats. Behav.Neurosci. 107:981-987.

Poltyrev T, Keshet G, Kay G, and Weinstock M (1996) Role of experimental conditions in determining differences in exploratory behavior of prenatally stressed rats. Dev.Psychobiol. 29:453-462. 
Popova NK, Avgustinovich DF, Kolpakov VG, and Plyusnina IZ (1998) Specific $\left[{ }^{3} \mathrm{H}\right] 8$ OH-DPAT binding in brain regions of rats genetically predisposed to various defense behavior strategies. Pharmacol.Biochem.Behav. 59:793-797.

Potter E, Sutton S, Donaldson C, Chen R, Perrin M, Lewis K, Sawchenko PE, and Vale W (1994) Distribution of corticotropin-releasing factor receptor mRNA expression in the rat brain and pituitary. Proc.Natl.Acad.Sci.USA 91:8777-8781.

Raadsheer FC, Hoogendijk WJG, Stam FC, Tilders FJH, and Swaab DF (1994) Increased numbers of corticotropin-releasing hormone expressing neurons in the hypothalamic paraventricular nucleus of depressed patients. Neuroendocrinology 60:436-444.

Radja F, Laporte AM, Daval G, Verge D, Gozlan H, and Hamon M (1991) Autoradiography of Serotonin Receptor Subtypes in the Central Nervous System. Neurochem.Int. 18:1-15.

Ramboz S, Oosting R, Amara DA, Kung HF, Blier P, Mendelsohn M, Mann JJ, Brunner D, and Hen R (1998) Serotonin receptor 1A knockout: An animal model of anxiety-related disorder. Proc.Natl.Acad.Sci.USA 95:14476-14481.

Reese WG, Angel C, and Newton JE (1984) Immobility reactions: a modified classification. Pavlov.J.Biol.Sci. 19:137-143.

Remy SM, Schreiber R, Dalmus M, and De Vry J (1996) Somatodendritic 5-HT $1 \mathrm{~A}$ receptors are critically involved in the anxiolytic effects of 8-OH-DPAT.

Psychopharmacol.(Berl.) 125:89-91.

Reyes TM and Coe CL (1997) Prenatal manipulations reduce the proinflammatory response to a cytokine challenge in juvenile monkeys. Brain Res. 769:29-35.

Richardson R and Elsayed H (1998) Shock sensitization of startle in rats: the role of contextual conditioning. Behav.Neurosci. 112:1136-1141.

Rogan MT, Stäubli UV, and LeDoux JE (1997) Fear conditioning induces associative longterm potentiation in the amygdala. Nature 390:604-607.

Rosen JB, Hitchcock JM, Sananes CB, Miserendino MJD, and Davis M (1991) A direct projection from the central nucleus of the amygdala to the acoustic startle pathway: Anterograde and retrograde tracing studies. Behav.Neurosci. 105:817-825.

Rothberg AD and Lits B (1991) Psychosocial support for maternal stress during pregnancy: effect on birth weight. Am.J.Obstet.Gynecol.403-407.

Sajdyk TJ, Schober DA, Gehlert DR, and Shekhar A (1999) Role of corticotropin-releasing factor and urocortin within the basolateral amygdala of rats in anxiety and panic responses. Behav.Brain Res. 100:207-215. 
Sapolsky RM and McEwen BS (1985) Down-regulation of neural corticosterone receptors by corticosterone and dexamethasone. Brain Res. 339:161-165.

Sarvey JM, Burgard EC, and Decker G (1989) Long-term potentiation: studies in the hippocampal slice. J.Neurosci.Meth. 28:109-124.

Scatton B, Zivkovic B, and Dedek J (1980) Antidopaminergic properties of yohimbine. J.Pharmacol.Exp.Therap. 215:494-499.

Schafe GE and LeDoux JE (2000) Memory Consolidation of Auditory Pavlovian Fear Conditioning Requires Protein Synthesis and Protein Kinase A in the Amygdala.

J.Neurosci. 20:RC96.

Schneider M (1992) Prenatal stress exposure alters postnatal behavioral expression under conditions of novelty challenge in rhesus monkey infants. Dev.Psychobiol. 25:529-540.

Schneider M (1993) Repeated scioal stress durng pregnancy impairs neuromotor development of the primate infant. Dev.Behav.Pediatr. 14:81-87.

Schulz D and Canbeyli R (1999) Freezing behavior in BNST-lesioned Wistar rats. Ann.N.Y.Acad.Sci. 877:728-731.

Sherman JE and Kalin NH (1988) ICV-CRH alters stress-induced freezing behavior without affecting pain sensitivity. Pharmacol.Biochem.Behav. 30:801-807.

Skofitsch G and Jacobowitz DM (1985) Distribution of corticotropin releasing factor-like immunoreactivity in the rat brain by immunohistochemistry and radioimmunoassay: Comparison and characterization of ovine and rat/human CRF antisera. Peptides 6:319336.

Smith DJ, Monroe PJ, Kirk HR, Crisp T, and Smith DL (1996) Pharmacological evaluation of neuronal mechanisms involved in spinal antinociception may be confounded by a lack of drug specificity: an analysis using opioid and monoaminergic interactions. Analgesia 2:3141.

Smith MA and Nemeroff CB (1988) Preclinical and clinical evidence for the involvement of corticotropin-releasing factor in the pathogenesis of depression. Adv.Exp.Med.Biol. 245:479-487.

Smythe JW, McCormick CM, and Meaney MJ (1996) Median eminence corticotrophinreleasing hormone content following prenatal stress and neonatal handling. Brain Res.Bull. 40:195-199.

Sontag LW (1941) The Significance of Fetal Environmental Differences. Am.J.Obstet.Gynecol. 42:996-1003.

Steckler T and Holsboer F (1999) Corticotropin-releasing hormone receptor subtypes and emotion. Biol.Psychiatry 46:1480-1508. 
Stenzel-Poore MP, Duncan JE, Rittenberg MB, Bakke AC, and Heinrichs SC (1996) CRH overproduction in transgenic mice - Behavioral and immune system modulation.

Ann.N.Y.Acad.Sci. 780:36-48.

Stiedl O, Misane I, Spiess J, and Ogren SO (2000) Involvement of the 5-HT1A receptors in classical fear conditioning in C57BL/6J mice. J.Neurosci. 20:8515-8527.

Stitzel RE (1972) Stress and strain--Factors influencing drug metabolism.

Biochem.Pharmacol. 21:755-760.

Stitzel RE and Furner RL (1967) Stress-induced alterations in microsomal drug metabolism in the rat. Biochem.Pharmacol. 16:1489-1494.

Stott DH (1957) Physical and Mental handicaps following a disturbed pregnancy. Lancet 1006-1012.

Stott DH (1973) Follow-up study from birth of the effects of prenatal stress.

Develop.Med.Child Neurol. 15:770-787.

Stott DH and Latchford SA (1976) Prenatal antecedants of child health, development, and behavior. J.Am.Acad.Child.Psychiat 15:161-191.

Suchecki D and Palermo NJ (1991) Prenatal stress and emotional response of adult offspring. Physiol.Behav. 49:423-426.

Sugino N, Nakamura Y, Okuno N, Shimamura K, Teyama T, Ishimatsu M, and Kato H (1994) Effects of restraint stress on luteal function in rats during mid- pregnancy. J.Reprod.Fertil. 101:23-26.

Svensson L (1985) Effects of 8-OH-DPAT, lisuride and some ergot-related compounds on the acoustic startle response in the rat. Psychopharmacol.(Berl.) 85:469-475.

Swanson LW, Sawchenko PE, Rivier J, and Vale W (1983) Organization of ovine corticotropin-releasing factor immunoreactive cells and fibers in the rat brain: An immunohistochemical study. Neuroendocrinology 36:165-186.

Swiergiel AH, Takahashi LK, and Kalin NH (1993) Attenuation of stress-induced behavior by antagonism of corticotropin-releasing factor receptors in the central amygdala in the rat. Brain Res. 623:229-234.

Sze PY, Neckers L, and Towle AC (1976) Glucocorticoids as a regulatory factor for brain tryptophan hydroxylase. J.Neurochem. 26:169-173.

Szuran TF, Pliska V, Pokorny J, and Welzl H (2000) Prenatal stress in rats: effects on plasma corticosterone, hippocampal glucocorticoid receptors, and maze performance. Physiol.Behav. 71:353-362. 
Takahashi LK (1994) Organizing action of corticosterone on the development of behavioral inhibition in the preweanling rat. Develop.Brain Res. 81:121-127.

Takahashi LK, Baker EW, and Kalin NH (1990) Ontogeny of behavioral and hormonal responses to stress in prenatally stressed male rat pups. Physiol.Behav. 47:357-364.

Takahashi LK, Haglin C, and Kalin NH (1992a) Prenatal stress potentiates stress-induced behavior and reduces the propensity to play in juvenile rats. Physiol.Behav. 51:319-323.

Takahashi LK, Turner JG, and Kalin NH (1992b) Prenatal stress alters brain catecholaminergic activity and potentiates stress-induced behavior in adult rats. Brain Res. 574:131-137.

Takahashi LK and Kalin NH (1991) Early developmental and temporal characteristics of stress- induced secretion of pituitary-adrenal hormones in prenatally stressed rat pups. Brain Res. 558:75-78.

Takahashi LK and Kim H (1995) Relative contributions of pituitary-adrenal hormones to the ontogeny of behavioral inhibition in the rat. Physiol.Behav. 57:711-716.

Takao K, Nagatani T, Kitamura Y, and Yamawaki S (1997) Effects of corticosterone on 5HT1A and 5-HT2 receptor binding and on the receptor-mediated behavioral responses of rats. Eur.J.Pharmacol. 333:123-128.

Tanimura SM and Watts AG (1998) Corticosterone can facilitate as well as inhibit corticotropin-releasing hormone gene expression in the rat hypothalamic paraventricular nucleus. Endocrinology 139:3830-3836.

Tavares MA and Silva MC (1996) Differential effects of prenatal exposure to cocaine and amphetamine on growth parameters and morphometry of the prefrontal cortex in the rat. Ann.N.Y.Acad.Sci. 801:256-273.

Thompson WR (1957) Influence of prenatal maternal anxiety on emotionality in young rats. Science 125:698-699.

Tork I (1990) Anatomy of the serotonergic system. Ann.N.Y.Acad.Sci. 600:9-35.

Trillat AC, Malagie I, Mathe-Allainmat M, Anmela MC, Jacquot C, Langlois M, and Gardier AM (1998) Effects of WAY 100635 and (-)-5-Me-8-OH-DPAT, a novel 5-HT1A receptor antagonist, on 8-OH-DPAT responses. Eur.J.Pharmacol. 347:41-49.

Vale W, Spiess J, and Rivier J (1981) Characterization of a 41 amino acid residue ovine hypothalamic peptide that stimulates the secretion of corticotropin and beta-endorphin. Science 213:1394-1397.

Vallee M, Maccari S, Dellu F, Simon H, Le Moal M, and Mayo W (1999) Long-term effects of prenatal stress and postnatal handling on age-related glucocorticoid secretion and cognitive performance: a longitudinal study in the rat. Eur.J.Neurosci. 11:2906-2916. 
Van Os J and Selten JP (1998) Prenatal exposure to maternal stress and subsequent schizophrenia - The May 1940 invasion of The Netherlands. Br.J.Psychiatry 172:324-326.

Verge D, Daval G, Marcinkiewicz M, Patey A, El Mestikawy S, Gozlan H, and Hamon M (1986) Quantitative autoradiography of multiple 5-HT1 receptor subtypes in the brain of control or 5,7-dihydroxytryptamine-treated rats. J.Neurosci. 6:3474-3482.

Vertes RP and Kocsis B (1994) Projections of the Dorsal Raphe Nucleus to the Brainstem: PHA-L Analysis in the Rat. J.Comp.Neurol. 340:11-26.

Wadhwa PD, Sandman CA, Porto M, Dunkel-Schetter C, and Garite TJ (1993) The association between prenatal stress and infant birth weight and gestational age at birth: a prospective investigation. Am.J.Obstet. Gynecol. 169:858-865.

Wakshlak A and Weinstock M (1990) Neonatal handling reverses behavioral abnormalities induced in rats by prenatal stress. Physiol.Behav. 48:289-292.

Walker DL, Cassella JV, Lee YG, De Lima TCM, and Davis M (1997) Opposing roles of the amygdala and dorsolateral periaqueductal gray in fear-potentiated startle.

Neurosci.Biobehav.Rev. 21:743-753.

Ward AJ (1991) Prenatal stress and child psychopathology. Child Psychiat.Human Develop. 22:97-110.

Ward HE, Johnson EA, Goodman IJ, Birkle DL, Cottrell DJ, and Azzaro AJ (1998) Corticotropin-releasing factor and defensive withdrawal: Inhibition of monoamine oxidase prevents habituation to chronic stress. Pharmacol.Biochem.Behav. 60:209-215.

Ward HE, Johnson EA, Salm AK, and Birkle DL (2000) Effects of prenatal stress on defensive withdrawal behavior and corticotropin releasing factor systems in rat brain. Physiol.Behav. 70:359-366.

Ward I and Weisz J (1984) Differential effects of maternal stress on circulating levels of corticosterone, progesterone, and testosterone, in male and female rat fetuses and their mothers. Endocrinology 114:1635-1644.

Ward IL (1984) The prenatal stress syndrome: Current status. pne 9:3-11.

Wehmer F and Jen KL (1978) The effects of litter size during gestation and lactation on rat development prior to weaning. Dev.Psychobiol. 11:353-360.

Weinstock M (1997) Does prenatal stress impair coping and regulation of hypothalamicpituitary-adrenal axis? Neurosci.Biobehav.Rev. 21:1-10.

Weinstock M, Fride E, and Hertzberg R (1988) Prenatal stress effects on functional development of the offspring. Prog.Brain Res. 73:319-331. 
Weinstock M, Poltyrev T, Schorer-Apelbaum D, Men D, and McCarty R (1998) Effect of prenatal stress on plasma corticosterone and catecholamines in response to footshock in rats. Physiol.Behav. 64:439-444.

Weiss JM (1997) Does decreased sucrose intake indicate loss of preference in CMS model? Psychopharmacol.(Berl.) 134:368-370.

Welberg LA, Seckl JR, and Holmes MC (2000) Inhibition of 11 beta-hydroxysteroid dehydrogenase, the foeto-placental barrier to maternal glucocorticoids, permanently programs amygdala GR mRNA expression and anxiety-like behaviour in the offspring. Eur.J.Neurosci. 12:1047-1054.

White DA and Birkle DL (2001) The differential effects of prenatal stress in rats on the acoustic startle reflex under baseline conditions and in response to anxiogenic drugs. Psychopharmacol.(Berl.) 154:169-176.

Williams MT, Davis HN, McCrea AE, Long S, and Hennessy MB (1999) Changes in the hormonal concentrations of pregnant rats and their fetuses following multiple exposures to a stressor during the third trimester. Neurotoxicol.Teratol. 21:403-414.

Williams MT, Hennessy MB, and Davis HN (1998) Stress during pregnancy alters rat offspring morphology and ultrasonic vocalizations. Physiol.Behav. 63:337-343.

Willner P (1997) Validity, reliability and utility of the chronic mild stress model of depression: a 10 year review and evaluation. Psychopharmacol.(Berl.) 134:319-329.

Willner P, Benton D, Brown E, Cheeta S, Davies G, Morgan J, and Morgan M (1998) "Depression" increases "craving" for sweet rewards in animal and human models of depression and craving. Psychopharmacol.(Berl.) 136:272-283.

Willner P, Moreau J-L, Nielsen CK, Papp M, and Sluzewska A (1996) Decreased hedonic responsiveness following chronic mild stress is not secondary to loss of body weight. Physiol.Behav. 60:129-134.

Winter JC and Rabin RA (1992) Yohimbine as a serotonergic agent: evidence from receptor binding and drug discrimination. J.Pharmacol.Exp.Ther. 263:682-689.

Wise DA and Eldred NL (1986) Maternal social stress disrupts reproduction of hamsters drinking high-calorie fluids. Pharmacol.Biochem.Behav. 25:449-456.

Wood M, Chaubey M, Atkinson P, and Thomas DR (2000) Antagonist activity of metachlorophenylpiperazine and partial agonist activity of 8-OH-DPAT at the 5-HT(7) receptor. Eur.J.Pharmacol. 396:1-8.

Yang K (2000) Placental 11 beta-hydroxysteroid dehydrogenase: barrier to maternal glucocorticoids. Rev.Reprod. 2:129-132. 
Young LJ (1999) Frank A. Beach Award. Oxytocin and vasopressin receptors and speciestypical social behaviors. Horm.Behav. 36:212-221.

Zarrow MX, Philpott JE, and Denenberg VH (1970) Passage of c-14-corticosterone from the rat mother to the foetus and neonate. Nature 226:1058-1059.

Zelena D, Haller J, Halász J, and Makara GB (1999) Social stress of variable intensity: Physiological and behavioral consequences. Brain Res.Bull. 48:297-302.

Zhang Y, Raap DK, Garcia F, Serres F, Ma Q, Battaglia G, and Van de Kar LD (2000) Long-term fluoxetine produces behavioral anxiolytic effects without inhibiting neuroendocrine responses to conditioned stress in rats. Brain Res. 855:58-66.

Zhong P and Ciaranello RD (1995) Transcriptional regulation of hippocampal 5-HT $1 \mathrm{a}$ receptors by corticosteroid hormones. Mol.Brain Res. 29:23-34. 